\title{
$s$-wave charmed baryon resonances from a coupled-channel approach with heavy quark symmetry
}

\author{
C. García-Recio, ${ }^{1}$ V. K. Magas, ${ }^{2}$ T. Mizutani, ${ }^{3}$ J. Nieves, ${ }^{4}$ A. Ramos, ${ }^{2}$ L. L. Salcedo, ${ }^{1}$ and L. Tolos ${ }^{5,6}$ \\ ${ }^{1}$ Departamento de Física Atómica, Molecular y Nuclear, Universidad de Granada, E-18071 Granada, Spain \\ ${ }^{2}$ Departament d'Esctructura i Constituents de la Matèria and Institut de Ciències del Cosmos, Universitat de Barcelona, E-08028 \\ Barcelona, Spain \\ ${ }^{3}$ Department of Physics, Virginia Polytechnic Institute and State University, Blacksburg, Virginia 24061, USA \\ ${ }^{4}$ Instituto de Física Corpuscular (centro mixto CSIC-UV), Institutos de Investigación de Paterna, Aptdo. 22085, 46071, Valencia, \\ Spain \\ ${ }^{5}$ Frankfurt Institute for Advanced Studies, Goethe-Universität, 60438 Frankfurt am Main, Germany \\ ${ }^{6}$ Theory Group, KVI, University of Groningen, Zernikelaan 25, 9747 AA Groningen, The Netherlands
} (Received 15 July 2008; published 5 March 2009)

\begin{abstract}
We study charmed baryon resonances that are generated dynamically within a unitary meson-baryon coupled-channel model that treats the heavy pseudoscalar and vector mesons on equal footing as required by heavy-quark symmetry. It is an extension of recent SU(4) models with $t$-channel vector-meson exchanges to an SU(8) spin-flavor scheme, but differs considerably from the SU(4) approach in how the strong breaking of the flavor symmetry is implemented. Some of our dynamically generated states can be readily assigned to recently observed baryon resonances, while others do not have a straightforward identification and require the compilation of more data as well as an extension of the model to $d$-wave meson-baryon interactions and $p$-wave coupling in the neglected $s$ - and $u$-channel diagrams. Of several novelties, we find that the $\Lambda_{c}(2595)$, which emerged as a $N D$ quasibound state within the SU(4) approaches, becomes predominantly a $N D^{*}$ quasibound state in the present $\mathrm{SU}(8)$ scheme.
\end{abstract}

DOI: 10.1103/PhysRevD.79.054004

PACS numbers: 14.20.Lq, 11.10.St, 12.38.Lg, 14.40.Lb

\section{INTRODUCTION}

In hadron physics establishing whether a resonance has the usual $q \bar{q}$ or $q q q$ structure or better qualifies as being dynamically generated through the multiple scattering of their meson and/or baryon components, has been an active topic of research. In recent years, the introduction of unitarity constraints in coupled channels methods has led to extensions of the chiral effective theories that can be applied at much higher energies and in the vicinity of resonances [1]. In the particular case of baryons, several resonances are firm candidates to be identified with states generated dynamically from the interaction of mesons of the $0^{-}$octet Goldstone bosons with the $1 / 2^{+}$octet groundstate baryons [2-20]. Then interesting predictions such as the two-pole nature of the $\Lambda(1405)[9,13,21-25]$ have found experimental confirmation [26,27], as discussed in Ref. [28]. Later a number of works has also been devoted to the study of dynamically generated $J^{P}=3 / 2^{-}$states [2939]. Early works considered the interaction of pseudoscalar $0^{-}$mesons with the baryons of the $3 / 2^{+}$decuplet. The incorporation of vector mesons into the above-mentioned coupled-channel picture was pursued in studies of axial $1^{+}$ meson resonances of Refs. [40,41], but until very recently those degrees of freedom have not been considered in the baryon-meson sector. In Ref. [37] the $\left(8_{3}, 1_{3}\right)^{1}$ nonet of lowest-lying vector meson $(\rho(770), K(890), \bar{K}(890)$,

\footnotetext{
${ }^{1}$ Here and below we use the notation $\mu_{2 J+1}$, for the SU(3) multiplet $\mu$ of $\operatorname{spin} J$.
}

$\omega(780)$ and $\phi(1020))$ degrees of freedom was incorporated into the Weinberg-Tomozawa (WT) meson-baryon chiral Lagrangian by using a scheme that relies on the SU(6) spin-flavor symmetry. The corresponding Bethe-Salpeter (BS) equation successfully reproduces the previous $\mathrm{SU}(3)$ flavor WT results for the lowest-lying $s$-and $d$-wave, negative parity baryon resonances $(N(1535), N(1650), \Lambda(1390)$, $\Lambda(1405), \Lambda(1520), \Lambda(1690), \Lambda(1670), \Xi(1620), \Xi(1690), \cdots)$. It also provides some information on the dynamics of the heavier ones such as $\Lambda(1800)$ or $\Lambda(2325)$, which have sizable $N \bar{K}^{*}$ and $\Lambda \omega$ couplings, respectively $[38,39]$.

Recently, the attention has turned toward studying resonances with charm degrees of freedom, motivated by the discovery of quite a few new states, as reported by the CLEO, Belle, and BABAR Collaborations [42-47]. At first sight, the physics in the charm $C=1$ sector bears a strong resemblance to the phenomenology seen in the $\bar{K} N$ dynamics upon replacing an $s$ quark by a $c$ quark. ${ }^{2}$ This is reinforced by an apparent similarity between the two $I=0$ $s$-wave $\Lambda(1405)$ and $\Lambda_{c}(2595)$ resonances. The mass of the former lies in between the $\pi \Sigma$ and $\bar{K} N$ channel thresholds, to which it couples strongly. The $\Lambda_{c}(2595)$ lies below the $D N$ and just slightly above the $\pi \Sigma_{c}$ thresholds. This similarity was exploited in the first exploratory work [48] where free space amplitudes were constructed from a set of

\footnotetext{
${ }^{2}$ Besides kinematical similarities (open and closed channels near the resonance mass ...), the underlying dynamics might be different as a strange quark is related to chiral symmetry and a charm quark to heavy-quark symmetry, as we will discuss below.
} 
separable coupled-channel interactions obtained from chirally motivated Lagrangians upon replacing the $s$ quark by the $c$ quark. The $I=0 \Lambda_{c}(2595)$ was generated as a $D N$ $s$-wave resonant state of binding energy $\approx 200 \mathrm{MeV}$ with a width of $\approx 3 \mathrm{MeV}$. While giving the first indication that the $\Lambda_{c}(2595)$ could have a dynamical origin, this work ignored the strangeness degree of freedom due to its very construction. Therefore, the $\pi$ and $K$ (Goldstone) mesons were not treated on an equal footing, and the role of some channels that would appear from the corresponding SU(4) meson and baryon multiplets, such as $D_{s} \Lambda, D_{s} \Sigma, K \Xi_{c}$, and $K \Xi_{c}^{\prime}$, was ignored. A different approach, which respected the proper symmetries, was attempted in Ref. [49]. There, charmed baryon resonances were generated dynamically from the scattering of Goldstone bosons off the ground-state $J^{P}=1 / 2^{+}$charmed baryons. The $C=$ $1, S=I=0$ resonance found at $2650 \mathrm{MeV}$ was identified with the $\Lambda_{c}(2595)$ in spite of the fact that, due to the strong coupling to the $\pi \Sigma_{c}$ states, its width came out to be more than 20 times larger than the experimental width of about $4 \mathrm{MeV}$. The trouble with this model is that, due to its very construction, it does not account for the coupling to the $D N$ channel, which contributes strongly to generating the narrow $\Lambda_{c}(2595)$ according to Ref. [48]. A substantial improvement came in a recent work [50] in which the abovementioned shortcomings have been overcome by exploiting the universal vector-meson coupling hypothesis to break the SU(4) symmetry in a convenient and welldefined manner. This is done by a $t$-channel exchange of vector mesons between pseudoscalar mesons and baryons in such a way that chiral symmetry is preserved in the light meson sector, while the interaction is still of the WT type. We note that in the subsequent sections this approach is referred as TVME (t-channel vector-meson exchange model). The model generates a narrow $C=1, I=0$ resonance that is identified with the $\Lambda_{c}(2595)$, together with other resonances in other strangeness-charm sectors with $J=1 / 2^{-}$. An extension of the model to $d$-wave $J=3 / 2^{-}$ resonances, generated by the interactions of pseudoscalar mesons with baryons of the $J=3 / 2^{+} \mathrm{SU}(4)$ multiplet, was developed in Ref. [51]. Finally, some modifications over the model in Ref. [50], both in the kernel and in the regularization scheme, were implemented in Ref. [52], obtaining qualitatively similar results to the earlier approaches. In all these works the zero-range limit in the $t$-channel exchange of vector mesons is identified as the driving force for the $s$-wave scattering of pseudoscalar mesons off the baryon ground states, and is used to carry out a coupled-channel analysis.

A serious limitation of this SU(4) TVME model is that, whereas the pseudoscalar mesons $D$ and $D_{s}$ are included in the coupled-channel dynamics, their vector partners $D^{*}$ and $D_{s}^{*}$ are completely left out. This is not justified from the point of view of heavy-quark symmetry (HQS), which is a proper QCD spin-flavor symmetry [53-55] when the quark masses become much larger than the typical confinement scale, $\Lambda_{\mathrm{QCD}}$.

HQS predicts that all types of spin interactions vanish for infinitely massive quarks: the dynamics is unchanged under arbitrary transformations in the spin of the heavy quark $(Q)$. The spin-dependent interactions are proportional to the chromomagnetic moment of the heavy quark, hence are of the order of $1 / m_{Q}$. The total angular momentum $\vec{J}$ of the hadron is a conserved quantity, and the spin of the heavy quark $\vec{S}_{Q}$ is also conserved in the $m_{Q} \rightarrow \infty$ limit. Consequently, the spin of the light degrees of freedom $\vec{S}_{l}=$ $\vec{J}-\vec{S}_{Q}$ is conserved in that limit. Thus, heavy hadrons come in doublets (unless $s_{l}=0$, with $\vec{S}_{l}^{2}=s_{l}\left(s_{l}+1\right)$ ) containing states with total spin $j_{ \pm}=s_{l} \pm 1 / 2$ obtained by combining the spin of the light degrees of freedom with the spin of the heavy quark $s_{Q}=1 / 2$. These doublets are degenerate in the $m_{Q} \rightarrow \infty$ limit. This is the case for the ground-state mesons $D$ and $D^{*}$ or $D_{s}$ and $D_{s}^{*}$, which are composed of a charm quark with $s_{Q}=1 / 2$ and light degrees of freedom with $s_{l}=1 / 2$, forming a multiplet of hadrons with spin 0 and 1 and negative parity. The entire multiplet of degenerate states should be treated in any HQS inspired formalism as a single field that transforms linearly under the heavy-quark symmetries [54,55]. For finite charm quark mass, the pseudoscalar and vector $D$ meson masses differ in just about one pion mass, ${ }^{3}$ even less for the strange charmed mesons, thus it is reasonable to expect, for instance, that the coupling $D N-D^{*} N$ might play an important role, and could well be bigger than that played by some of the other channel couplings considered in the TVME model, say, the $D N-\Lambda_{c} \eta$.

The mass splitting between pseudoscalar and vector mesons is comparatively larger in the light sectors, for example, between the $\pi$ and $\rho$ mesons or the $K$ and the $K^{*}$. This appears to justify the usual approximation of ignoring vector degrees of freedom in the SU(3) coupledchannel studies in $s$-wave meson $\left(8_{1}\right)$-meson $\left(8_{1}\right)$ and meson $\left(8_{1}\right)$-baryon $\left(8_{2}, 10_{4}\right)$ scattering and resonances, within different renormalization schemes. Nevertheless, as already mentioned, vector mesons have been incorporated in the WT meson-baryon chiral Lagrangian [37$39,56]$ through a scheme that treats the six states of a light quark ( $u, d$ or $s$ with spin up, $\uparrow$, or down, $\downarrow$ ) as equivalent, leading to an SU(6) symmetric scheme. Here, we will extend this model to SU(8) spin-flavor symmetry to account also for the charm quark degree of freedom. Therefore, compared to the TVME approach, our coupled-channel analysis will consider the additional role of vector mesons $\left(D^{*}, D_{s}^{*}, \rho, K^{*}, \ldots\right)$. Moreover, both models differ on how they implement the strong breaking of the $\mathrm{SU}(4)$ flavor symmetry observed in nature.

We should clarify here that the extension of the WT interaction to vector mesons [37] and to flavor SU(4) is a model. Chiral symmetry is a symmetry of the light quark

\footnotetext{
${ }^{3}$ One can easily deduce that $m_{D}-m_{D^{*}}=\mathcal{O}\left(\frac{1}{m_{D}+m_{D^{*}}}\right)$
} 
sector, i.e. at best for flavor SU(3), but not for SU(4). Chiral symmetry breaking fixes model independently the strength of the lowest order interaction between Goldstone bosons and other hadrons (here baryons), namely, the WT interaction. However, chiral symmetry does not fix the interaction between vector mesons and baryons. On the other hand, HQS connects vector and pseudoscalar mesons which contain charm quarks. It does not tell anything about mesons made out of light quarks. Nevertheless, it is clearly appealing to have a model for four flavors and for pseudoscalar and vector mesons, which reduces to the WT interaction in the sector where Goldstone bosons are involved and which incorporates heavy-quark symmetry in the sector where charm quarks are involved. The model assumption in the present extension does not appear to be easy to justify, but we want to try it based on the reasonable outcome of the SU(6) extension in the three-flavor sectors on the one hand, and formal plausibility of how the SU(4) WT interaction in the charmed pseudoscalar meson-SU(3) baryon interaction did come out in the vector-meson exchange picture as discussed in the TVME approach, on the other hand. We just want to fuse the two approaches. Note than in our case, we improve on previous models since we incorporate HQS in the charm sector.

In this exploratory work, we have studied only nonstrange single charmed resonances. Our approach should work better close to the relevant thresholds, and, in particular, to describe the lowest-lying resonances in each of the examined $I J$ (meson-baryon isospin and total angular momentum) sectors. Actually, working near threshold is the only justification to ignore the contribution of $d$-wave meson-baryon interactions and of $p$-wave couplings, through the neglected $s$-and $u$-channel diagrams, which otherwise might play an important role. These contributions have been also traditionally ignored in the previous successful SU(3) flavor studies of lowest-lying charmless $s$-and $d$-wave resonances [2-5,7-19,29-39], which we aim to extend to the charm sector.

The present work is organized as follows. In Sec. II, we give the details of our SU(8) extension of the WT mesonbaryon Lagrangian. The basis of states and the general form of the Lagrangian are presented in Sec. II A, while the symmetry breaking effects are discussed in Sec. II B. The unitarization method and renormalization procedure are described in Sec. III. Our SU(8) results are discussed in Sec. IV, while Sec. V is devoted to a comparison with results obtained in a reduced SU(4) model. A summary of our conclusions is presented in Sec. VI.

\section{SU(8) EXTENSION OF THE WT MESON- BARYON LAGRANGIAN}

\section{A. SU(8) symmetry}

Since we assume that the pure SU(4) (flavor) transformations commute with the pure $\mathrm{SU}(2)$ (spin) transformations, it follows that an $\mathrm{SU}(8)$ multiplet can be decomposed into SU(4) multiplets each with definite total spin. With the inclusion of spin there are 64 quark-antiquark $(q \bar{q})$ states, and the irreducible reduction of the $\mathrm{SU}(8)$ group representation (denoting the SU(4) multiplets of dimensionality $\boldsymbol{n}$ and spin $J$ by $\boldsymbol{n}_{2 J+1}$ ) reads

$$
8 \otimes 8^{*}=63 \oplus 1=\underbrace{\left(15_{1} \oplus \mathbf{1 5}_{3} \oplus \mathbf{1}_{3}\right)}_{63} \oplus \mathbf{1}_{1} .
$$

Assuming that the lowest bound state is an $s$-state, and since the relative parity of a fermion-antifermion pair is odd, the SU(4) 15-plet of pseudoscalar $\left(D_{s}, D, K, \pi, \eta, \eta_{c}\right.$, $\left.\bar{K}, \bar{D}, \bar{D}_{s}\right)$ and the 16-plet of vector $\left(D_{s}^{*}, D^{*}, K^{*}, \rho, \omega\right.$, $\left.J / \Psi, \bar{K}^{*}, \bar{D}^{*}, \bar{D}_{s}^{*}, \phi\right)$ mesons are placed in the 63 representation. Note that the $\mathbf{6 3}$ allows nine light vector mesons but only eight $0^{-}$light mesons. A ninth $0^{-}$meson must go into the $\mathbf{1}$ of $\mathrm{SU}(8)$. We use pure $c \bar{c}$ wave functions for the charmonium states $\eta_{c}$ and $J / \Psi$, and the usual quark content, $\quad \eta=\frac{1}{\sqrt{6}}(u \bar{u}+d \bar{d}-2 s \bar{s}), \quad \eta^{\prime}=\frac{1}{\sqrt{3}}(u \bar{u}+d \bar{d}+s \bar{s})$, $\omega=\frac{1}{\sqrt{2}}(u \bar{u}+d \bar{d}), \phi=-s \bar{s}$, for the physical isoscalar light mesons. Such a specification induces some mixing between the isoscalar SU(4) mathematical states to build the physical states. Detailed spin-flavor wave functions specifying our conventions are given in Appendix A. Mesons of spin $J>1$ can be understood as states of the $q \bar{q}$ system with orbital angular momentum $L>0$, or molecular-type $q q \bar{q} \bar{q}$ meson-meson states.

In the case of baryons, with the inclusion of the spin, one finds 512 three quark states:

$$
\begin{aligned}
8 \otimes 8 \otimes 8= & 120 \oplus 56 \oplus 168 \oplus 168 \\
= & \underbrace{\left(20_{2} \oplus 20_{4}^{\prime}\right)}_{120} \oplus \underbrace{\left(4_{4} \oplus 20_{2}\right)}_{56} \\
& \oplus 2 \times \underbrace{\left(20_{2}^{\prime} \oplus 20_{4} \oplus 20_{2} \oplus 4_{2}\right)}_{168},
\end{aligned}
$$

where $\square$ and $\square \square$ are the 20 and $\mathbf{2 0}^{\prime}$ SU(4) representations, respectively. It is natural to assign the lowest-lying baryons to the $\mathbf{1 2 0}$ of $\mathrm{SU}(8)$. This is appropriate because in the light sector it can accommodate an octet of spin- $1 / 2$ baryons and a decuplet of spin-3/2 baryons, which are precisely the SU(3)-spin combinations of the low-lying baryon states $\left(N, \Sigma, \Lambda, \Xi\right.$ and $\left.\Delta, \Sigma^{*}, \Xi^{*}, \Omega\right)$. The remaining states in the $\mathbf{2 0}_{\mathbf{2}}$ and $\mathbf{2 0}_{\mathbf{4}}^{\prime}$ are completed with the charmed baryons $\Lambda_{c}, \Sigma_{c}, \Xi_{c}, \Xi_{c}^{\prime}, \Omega_{c}, \Xi_{c c}, \Omega_{c c}$ and $\Sigma_{c}^{*}$, $\Xi_{c}^{*}, \Omega_{c}^{*}, \Omega_{c c}^{*}, \Xi_{c c}^{*}, \Omega_{c c c}$, respectively. Quantum numbers of the charmed baryons are summarized in Table I. The $\mathbf{1 2 0}$ of $\mathrm{SU}(8)$ is totally symmetric, which allows the baryon to be made of three quarks in $s$ wave (the color wavefunction being antisymmetric).

Here, we will focus on the $s$-wave interaction between the lowest-lying meson (63) and the lowest-lying baryon (120) SU(8) multiplets at low energies. Note that at higher energies, higher partial waves would become important, so 
TABLE I. Summary of the quantum numbers and experimental masses of the baryons containing heavy quarks. $I$, and $S_{\text {light }}^{\pi}$ are the isospin, and the spin parity of the light degrees of freedom and $S, J^{P}$ are strangeness and the spin parity of the baryon $(l$ denotes a light quark of flavor $u$ or $d$ ). Isospin averaged experimental masses are taken from Ref. [57], with errors counting for the mass differences between the members of the same isomultiplet and the uncertainties quoted in Ref. [57].

\begin{tabular}{|c|c|c|c|c|c|c|}
\hline Baryon & $S$ & $J^{P}$ & $I$ & $S_{\text {light }}^{\pi}$ & Quark content & $M_{\text {exp }} \cdot[57](\mathrm{MeV})$ \\
\hline$\Lambda_{c}$ & 0 & $\frac{1}{2}^{+}$ & 0 & $0^{+}$ & $u d c$ & $2286.46 \pm 0.14$ \\
\hline$\Sigma_{c}$ & 0 & $\frac{1}{2}^{+}$ & 1 & $1^{+}$ & $l l c$ & $2453.6 \pm 0.7$ \\
\hline$\Sigma_{c}^{*}$ & 0 & $\frac{\frac{2}{3}}{2}+$ & 1 & $1^{+}$ & $l l c$ & $2518.0 \pm 0.5$ \\
\hline$\Xi_{c}$ & -1 & $\frac{1}{2}^{+}$ & $\frac{1}{2}$ & $0^{+}$ & $l s c$ & $2469.5 \pm 1.5$ \\
\hline$\Xi_{c}^{\prime}$ & -1 & $\frac{1}{2}+$ & $\frac{1}{2}$ & $1^{+}$ & $l s c$ & $2577 \pm 3$ \\
\hline$\Xi_{c}^{*}$ & -1 & $\frac{3}{2}+$ & $\frac{1}{2}$ & $1^{+}$ & $l s c$ & $2646.3 \pm 1.4$ \\
\hline$\Omega_{c}$ & -2 & $\frac{1}{2}^{+}$ & 0 & $1^{+}$ & $s s c$ & $2697.5 \pm 2.6$ \\
\hline$\Omega_{c}^{*}$ & -2 & $\frac{3}{2}+$ & 0 & $1^{+}$ & $s s c$ & \\
\hline$\Xi_{c c}$ & 0 & $\frac{1}{2}^{+}$ & $\frac{1}{2}$ & $\frac{1}{2}^{+}$ & $c c l$ & \\
\hline$\Xi_{c c}^{*}$ & 0 & $\frac{3}{2}+$ & $\frac{1}{2}$ & $\frac{1}{2}^{+}$ & $c c l$ & \\
\hline$\Omega_{c c}$ & -1 & $\frac{1}{2}^{+}$ & 0 & $\frac{1}{2}^{+}$ & ccs & \\
\hline$\Omega_{c c}^{*}$ & -1 & $\frac{3}{2}+$ & 0 & $\frac{1}{2}+$ & $\operatorname{ccs}$ & \\
\hline$\Omega_{c c c}^{*}$ & 0 & $\frac{3}{2}^{+}$ & 0 & - & $c c c$ & \\
\hline
\end{tabular}

a suitable treatment of spin-orbit effects in the $\mathrm{SU}(8)$ scheme should be considered. Now, assuming that the $s$-wave effective meson-baryon Hamiltonian is SU(8) invariant, and since the $\mathrm{SU}(8)$ decomposition of the product of the $\mathbf{6 3}$ and $\mathbf{1 2 0}$ representations yields

$$
63 \otimes 120=120 \oplus 168 \oplus 2520 \oplus 4752,
$$

we conclude that there are only four independent WignerEckart irreducible matrix elements (WEIME's) each of which being a function of the meson-baryon Mandelstam variable $s$. The WEIME's might be constrained by demanding that the $\mathrm{SU}(8)$ amplitudes for the scattering of the Goldstone $J^{P}=0^{-}$mesons of the pion octet off the $J^{P}=1 / 2^{+}$baryons of the nucleon octet reduce to those from $\mathrm{SU}(3)$ chiral symmetry. At leading order in the chiral expansion, these latter amplitudes are obtained from the WT Lagrangian which, besides hadron masses, only depends on the pion weak decay constant $f \simeq 90 \mathrm{MeV}$ in the chiral limit.

This procedure is a natural extension of the one derived in Ref. [37] for $\mathrm{SU}(6)$ to the spin-flavor $\mathrm{SU}(8)$ symmetry group. If one follows the arguments in this reference, it is clear that not all the SU(3) invariant interactions in the $\left(\mathbf{8}_{1}\right)$ meson $-\left(\mathbf{8}_{\mathbf{2}}\right)$ baryon sector can be extended to an SU(8) invariant interaction. This is easily understood because the number of independent SU(3) WEIME's to describe the $\left(\mathbf{8}_{\mathbf{1}}\right)$ meson $-\left(\mathbf{8}_{\mathbf{2}}\right)$ baryon interaction is six, as follows trivially from the $\mathrm{SU}(3)$ decomposition

$$
8 \otimes 8=1 \oplus 8_{s} \oplus 8_{a} \oplus 10 \oplus 10^{*} \oplus 27,
$$

whereas the $\mathrm{SU}(8)$ spin-flavor symmetry requires the knowledge of four WEIME's, as seen from Eq. (3). However, chiral symmetry (CS) at leading order is much more predictive than SU(3) symmetry, and it predicts the values of the various SU(3) WEIME's, which otherwise would be totally arbitrary functions of $s$. Indeed, the WT Lagrangian is not just $\mathrm{SU}(3)$ symmetric but also chiral $\left(\mathrm{SU}_{L}(3) \otimes \mathrm{SU}_{R}(3)\right)$ invariant. Symbolically, up to an overall constant, the WT interaction is

$$
\mathcal{L}_{\mathrm{WT}}=\operatorname{Tr}\left(\left[M^{\dagger}, M\right]\left[B^{\dagger}, B\right]\right) .
$$

This structure, dictated by CS, is most suitable to analyze in the $t$ channel. The mesons $M$ fall in the $\mathrm{SU}(3)$ representation $\mathbf{8}$, which is also the adjoint representation. The commutator $\left[M^{\dagger}, M\right]$ indicates a $t$-channel coupling to the $\mathbf{8}_{a}$ (antisymmetric) representation, thus

$$
\mathcal{L}_{\mathrm{WT}}=\left(\left(M^{\dagger} \otimes M\right)_{\mathbf{8}_{a}} \otimes\left(B^{\dagger} \otimes B\right)_{\mathbf{8}_{a}}\right)_{\mathbf{1}} .
$$

Note that from the group point of view there would be as many different $t$-channel $\mathrm{SU}(3)$ singlet Lagrangians as WEIME's.

For the SU(8) spin-flavor symmetry, the mesons $M$ fall in the 63, which is the adjoint representation and the baryons $B$ are found in the $\mathbf{1 2 0}$, which is fully symmetric, and the group reductions

$$
63 \otimes 63=1 \oplus 63_{s} \oplus 63_{a} \oplus 720 \oplus 945 \oplus 945^{*} \oplus 1232
$$$$
120 \otimes 120^{*}=1 \oplus 63 \oplus 1232 \oplus 13104
$$

lead ${ }^{4}$ to the total of four different $t$-channel SU(8) singlet couplings (that can be used to construct $s$-wave mesonbaryon interactions)

\footnotetext{
${ }^{4}$ The singlet representation $\mathbf{1}$ only appears in the reduction of the product of one representation by its complex-conjugate. The couplings of Eq. (8) arise because 1, 63, and $\mathbf{1 2 3 2}$ are selfcomplex conjugate representations.
} 


$$
\begin{gathered}
\left(\left(M^{\dagger} \otimes M\right)_{1} \otimes\left(B^{\dagger} \otimes B\right)_{1}\right)_{1}, \\
\left(\left(M^{\dagger} \otimes M\right)_{\mathbf{6 3}_{a}} \otimes\left(B^{\dagger} \otimes B\right)_{63}\right)_{1}, \\
\left(\left(M^{\dagger} \otimes M\right)_{\mathbf{6 3}_{s}} \otimes\left(B^{\dagger} \otimes B\right)_{63}\right)_{1}, \\
\left(\left(M^{\dagger} \otimes M\right)_{1232} \otimes\left(B^{\dagger} \otimes B\right)_{1232}\right)_{1},
\end{gathered}
$$

which match in number to that of the independent WEIME's expected from the group reduction of Eq. (3). To ensure that the SU(8) amplitudes will reduce to those deduced from the SU(3) WT Lagrangian in the $\left(\mathbf{8}_{1}\right)$ meson $-\left(\mathbf{8}_{2}\right)$ baryon subspace, we set all the couplings in Eq. (8) to be zero except for

$$
\mathcal{L}_{\mathrm{WT}}^{\mathrm{SU}(8)}=\left(\left(M^{\dagger} \otimes M\right)_{63_{a}} \otimes\left(B^{\dagger} \otimes B\right)_{63}\right)_{1},
$$

which is the natural and unique $\mathrm{SU}(8)$ extension of the usual SU(3) WT Lagrangian. ${ }^{5}$ The reduction of this Lagrangian to the $\mathrm{SU}(6)$ sector reproduces the $\mathcal{L}_{\mathrm{WT}}^{\mathrm{SU}(6)}$ found in Ref. [37]. The corresponding BS approximation, which we will discuss below, successfully reproduces the previous SU(3)-flavor WT results for the lowest-lying $s$-and $d$-wave negative parity baryon resonances obtained from scattering of the mesons of the pion octet off baryons of the nucleon octet and delta decuplet [38]. To compute the matrix elements of the $\mathrm{SU}(6) \mathrm{WT}$ interaction $\mathcal{L}_{\mathrm{WT}}^{\mathrm{SU}(6)}$, the SU(6)-multiplet coupling factors found in [58] were used in $[37,38]$. Here, because of the lack of these factors for the SU(8) group, we use quark model constructions of hadrons with field theoretical methods to express everything in tensor representations as described in Appendix A. Thus, we get the tree-level amplitudes (we use the convention $V=-\mathcal{L}$ )

$$
V_{a b}^{I J S C}(\sqrt{s})=D_{a b}^{I J S C} \frac{\sqrt{s}-M}{2 f^{2}}\left(\sqrt{\frac{E+M}{2 M}}\right)^{2},
$$

where the last factor is due to the spinor normalization convention: $\bar{u} u=\bar{v} v=1$, as in Refs. [14,22]. In the above expression IJSC are the meson-baryon isospin, total angular momentum, strangeness and charm quantum numbers, $M(E)$ the common mass [center-of-mass (CM) energy] of the baryons placed in the $\mathbf{1 2 0} \mathrm{SU}(8)$ representation, and $D^{I J S C}$ a matrix in the coupled-channel space. For instance, in the $J=3 / 2, I=2, S=0, C=1$ sector we consider 5 channels, $\Sigma_{c} \rho, \Delta D, \Sigma_{c}^{*} \pi \Delta D^{*}$, and $\Sigma_{c}^{*} \rho$, without including channels with double charmed baryons since they are much higher in energy. The $D$ matrices relevant for this work are presented in Tables XV, XVI, XVII, XVIII, XIX, XX, XXI, XXII, and XXIII of Appendix B. Note that we truncate the coupled-channel space by not including either double charmed baryon en-

\footnotetext{
${ }^{5}$ Alternatively, the unique spin-flavor symmetric extension of the WT Lagrangian is derived in [56] for arbitrary number of flavors and colors assuming only a nonrelativistic reduction for the baryons.
}

tries, or $\eta_{c}$, or $J / \Psi$ mesons, since in this work we are just interested in the charm $C=1$ sector. Double charmed baryons or $\eta_{c}$, or $J / \Psi$ mesons will contribute to this sector only in conjunction to another charmed hadron, and the threshold of the corresponding channel would be much higher in energy than those of channels involving a single charmed hadron.

\section{B. SU(8)-flavor symmetry breaking effects}

The SU(8) spin-flavor symmetry is strongly broken in nature. So we have taken into account mass breaking effects by adopting the physical hadron masses both in the tree-level interaction of Eq. (10) and in the evaluation of the kinematical thresholds of different channels.

Next, we have considered spin-flavor symmetry breaking effects due to the difference between the weak noncharmed and charmed, as well as pseudoscalar and vectormeson decay constants. These symmetry breaking effects are sizable because, for instance, the ratios $f_{D} / f_{\pi}$ of $f_{\rho} / f_{\pi}$ deviate from one, and actually they are of the order of 1.7. The pseudoscalar meson $(P)$ decay constants, $f_{P}$, are defined by

$$
\left\langle 0\left|\bar{q}_{1} \gamma^{\mu} \gamma_{5} q_{2}(0)\right| P(p)\right\rangle=-\mathrm{i} \sqrt{2} f_{P} p^{\mu}
$$

and vector meson $(V)$ decay constants, $f_{V}$, by

$$
\left\langle 0\left|\bar{q}_{1} \gamma^{\mu} q_{2}(0)\right| V(p, \epsilon)\right\rangle=\sqrt{2} m_{V} f_{V} \epsilon^{\mu},
$$

where $\bar{q}_{1}, q_{2}$ are the quark fields, $\epsilon_{\mu}$ is the polarization vector of the meson, and $m_{V}$ its mass. With the above definitions, HQS predicts $f_{D_{(s)}}=f_{D_{(s)}^{*}}$, up to $\Lambda_{\mathrm{QCD}} / m_{c}$ corrections [55], which guarantees that the normalizations of the coupling constants in Eqs. (11) and (12) are consistent. For light mesons there exist sizable corrections to the HQS-type relation $f_{P}=f_{V}$.

Within our present approach, besides the use of physical hadron masses, we further break the SU(8) symmetry of Eq. (10) by the replacement $f^{2} \rightarrow f_{a} f_{b}$, depending on the nature of the mesons involved. In Table II, we compiled the values of the decay constants used throughout this work. Whenever possible, we use the experimental values for the decay constants.

Taking into account all the $\mathrm{SU}(8)$ breaking effects stated above, our tree-level amplitudes now read

$$
\begin{aligned}
V_{a b}^{I J S C}(\sqrt{s})= & D_{a b}^{I J S C} \frac{2 \sqrt{s}-M_{a}-M_{b}}{4 f_{a} f_{b}} \\
& \times \sqrt{\frac{E_{a}+M_{a}}{2 M_{a}}} \sqrt{\frac{E_{b}+M_{b}}{2 M_{b}}},
\end{aligned}
$$

where $M_{a}\left(M_{b}\right)$ and $E_{a}\left(E_{b}\right)$ are, respectively, the mass and the CM energy of the baryon in the $a(b)$ channel. Though the way these flavor breaking effects have been introduced is intuitive, we should acknowledge here again that this constitutes a further assumption of our model, and that we 
can not establish a clear connection to any kind of first principles, as it is the case for all previous models.

We finish this section by comparing our present scheme with the one in TVME. The latter relies on SU(4) symmetry to construct the effective interaction between pseudoscalar mesons in the 16-plet with the $\mathbf{2 0}_{\mathbf{2}}$ and $\mathbf{2 0}$-plet representations of $J^{P}=1 / 2^{+}$and $J^{P}=3 / 2^{+}$baryons through a $t$-channel exchange of the 16-plet of vector mesons. Note that vector mesons do not enter in the $s$-channel coupled space. Then the universal vector-meson coupling hypothesis provides the global interaction strength among the above SU(4)-multiplets. However, deviations up to $50 \%$ from the universal value might be expected, as acknowledged in Ref. [50] from an analysis of the $D^{*} \rightarrow D \pi$ decay rate. Then, aided by the Kawarabayashi-Suzuki-Fayyazuddin-Riazudden relation, which is consistent with chiral symmetry at very low energy and momentum transfer, the resultant lowest order meson-baryon interaction in the $\mathrm{SU}(4)$ limit is found to take the WT form of Eq. (10) in the zero-range limit [52]. ${ }^{6}$ SU(4) symmetry is broken in the TVME model by using the physical hadron masses, without adopting different values for meson decay constants. The use of the physical masses for the pseudoscalar mesons and the baryons (external legs in the coupled-channel formalism of the TVME model) is analogous to the mass breaking effects included in the present scheme upon replacing $(\sqrt{s}-M)$ by $(2 \sqrt{s}-$ $\left.M_{a}-M_{b}\right) / 2$ in Eq. (10). However, the use of physical masses for the vector mesons in the $t$ channel in the TVME model leads to an additional symmetry breaking effect, which is different from those induced by the use of different meson decay constants in our scheme. For instance, let us consider the $\Sigma_{c} \pi \rightarrow \Sigma_{c} \pi, N D \rightarrow N D$, and $\Sigma_{c} \pi \rightarrow N D$ transitions. Within the TVME model the first two amplitudes are driven by the $t$-channel exchange of light vector mesons such as $\rho$, while the last one is by the exchange of a charmed $D^{*}$ vector meson. Thus, besides different SU(4) coupling coefficients, the last channel obtains an additional suppression factor of $\sim m_{\rho}^{2} / m_{D^{*}}^{2}=$ $1 / 6.8$ relative to the first two [52]. Within our scheme, these three channels will scale as $1 / f_{\pi}^{2}, 1 / f_{D}^{2}$, and $1 /\left(f_{D} f_{\pi}\right)$, respectively. Then on top of the matrix elements $\left(D_{a b}^{I J S C}\right)$ we obtain suppression factors $f_{\pi}^{2} / f_{D}^{2} \sim 1 / 2.9$ and $f_{\pi} / f_{D} \sim 1 / 1.7$ for the second and third amplitudes, respectively, relative to the first one. In summary, in the present work diagonal transitions involving charmed mesons are suppressed by about a factor of 3 with respect to the TVME SU(4) models [50,52], while the charmexchange amplitudes will not be suppressed by the mass factor $\sim m_{D^{*}}^{2} / m_{\rho}^{2}=6.8$ but only by a factor of about 2 due to the use of physical values for the decay constants. Thus, it is clear that the difference in the pattern of SU(4)

\footnotetext{
${ }^{6} \mathrm{~A}$ small $p$-wave contribution of the TVME model was neglected to reduce it to the WT form [52].
}

symmetry breaking in the two schemes discussed above is even qualitative.

\section{BS MESON-BARYON SCATTERING MATRIX}

We solve the coupled-channel BS equation with the interaction kernel determined by Eq. (13), which incorporates the SU(8) breaking effects discussed in Sec. II B. For a given IJSC sector, the solution for the coupled-channel $s$-wave scattering amplitude $T^{I J S C}(\sqrt{s})$, in the on-shell scheme $[5,8,9,22,66-68]$ is

$$
T^{I J S C}(\sqrt{s})=\frac{1}{1-V^{I J S C}(\sqrt{s}) G^{I J S C}(\sqrt{s})} V^{I J S C}(\sqrt{s}) .
$$

Here, $G^{I J S C}(\sqrt{s})$ is a diagonal matrix consisting of loop functions. The loop function for channel $i$ reads as

$$
\begin{aligned}
G_{i i}^{I J S C}(\sqrt{s})= & \mathrm{i} 2 M_{i} \int \frac{d^{4} q}{(2 \pi)^{4}} \frac{1}{(P-q)^{2}-M_{i}^{2}+i \varepsilon} \\
& \times \frac{1}{q^{2}-m_{i}^{2}+\mathrm{i} \varepsilon},
\end{aligned}
$$

where $M_{i}$ and $m_{i}$ are the masses of the baryon and meson in the channel, respectively. This quantity is logarithmically divergent, hence must be regularized. For example, the regularization may be done by one-subtraction at the subtraction point $\sqrt{s}=\mu_{i}^{I J S C}$ such that

$$
G_{i i}^{I J S C}\left(\sqrt{s}=\mu_{i}^{I J S C}\right)=0,
$$

with index $i$ running in the coupled-channel space. The TVME model of Refs. [50,51] uses the renormalization scheme (RS) of Eq. (16), and we adopt here its choice for the value of the subtraction point $\mu_{i}^{I J S C}$. It is taken to be independent of the spin $J$, then set identical within a given sector $I S C$ to $\sqrt{m_{\mathrm{th}}^{2}+M_{\mathrm{th}}^{2}}$, where $m_{\mathrm{th}}+M_{\mathrm{th}}$ is the mass of the lightest hadronic channel. For the SU(6) sector, this RS recovers previous results for the lowest-lying $1 / 2^{-}$and $3 / 2^{-}$baryon resonances appearing in the scattering of the octet of Goldstone bosons off the lowest baryon octet and decuplet $[5,8-10,13,22,23,29,30]$, and leads to new predictions for higher energy resonances. According to the authors of Refs. [50,51], such a choice guarantees an approximate crossing symmetry although such a claim appears somewhat dubious because crossing symmetry involves isospin mixtures, thus choosing an alternative subtraction point might lead to yet another reasonable result. Nevertheless, one should bear in mind an apparent correlation between the renormalization procedure and/or subtraction point, and the values for the meson decay constants used in the potential $V^{I J S C}$. For instance, it should be useful to mention the SU(6) results, which are somewhat complementary to the present approach in the choice of the subtraction points and meson decay constants in Ref. [38]. By taking a different value of the subtraction point for each channel $i$ of a given IJSC sector in the form 
$\mu_{i}^{I J S C}=\sqrt{M_{i}^{2}+m_{i}^{2}}$, the model gives a good agreement with a common Goldstone boson decay constant value of $90 \mathrm{MeV}$ (pion decay constant in the chiral limit), instead of using the different values quoted in Table II.

Given these uncertainties, in the present work we allow for slight changes in the choice of the subtraction point by introducing a parameter $\alpha$, such that

$$
\left(\mu^{I S C}\right)^{2}=\alpha\left(m_{\mathrm{th}}^{2}+M_{\mathrm{th}}^{2}\right),
$$

which will be fitted to data. In principle, $\alpha$ could have a different value in each IJSC sector. Unfortunately, we have insufficient empirical information. Therefore, we will adjust the value of $\alpha$ to reproduce the position of the wellestablished $\Lambda_{c}(2595)$ resonance with $I J S C=$

TABLE II. Meson decay constants. We use $\Gamma\left(\rho \rightarrow e^{+} e^{-}\right)=$ $7.04 \pm 0.06 \mathrm{KeV}, \Gamma\left(\omega \rightarrow e^{+} e^{-}\right)=0.60 \pm 0.02 \mathrm{KeV}, \Gamma(\phi \rightarrow$ $\left.e^{+} e^{-}\right)=1.27 \pm 0.04 \mathrm{KeV}, \quad \Gamma\left(\tau \rightarrow \rho \nu_{\tau}\right)=(5.71 \pm 0.07) \times$ $10^{-10} \mathrm{MeV}$, and $\Gamma\left(\tau \rightarrow K^{*} \nu_{\tau}\right)=(3.06 \pm 0.45) \times 10^{-11} \mathrm{MeV}$ from [57]. Besides, $G=1.1664 \times 10^{-11} \mathrm{MeV}^{-2}$ and $\alpha=$ $1 / 137.036$ are the Fermi and fine-structure constants, and $\cos \theta_{C}=0.974$, the cosine of the Cabibbo angle.

\begin{tabular}{lcc}
\hline \hline Decay constant & Exp. value $(\mathrm{MeV})$ & Theor. estimates $(\mathrm{MeV})$ \\
\hline$f_{\pi}$ & $92.4 \pm 0.1 \pm 0.3[57]$ & - \\
$f_{K}$ & $113.0 \pm 1.0 \pm 0.3[57]$ & - \\
$f_{\eta}$ & $\simeq 1.2 f_{\pi}[57]$ & - \\
$f_{\rho}$ & $\simeq 153^{\mathrm{a}}$ & - \\
$f_{K^{*}}$ & $\simeq 153^{\mathrm{b}}$ & - \\
$f_{\omega}$ & $\simeq 138^{\mathrm{c}}$ & - \\
$f_{\phi}$ & $\simeq 163^{\mathrm{d}}$ & - \\
$f_{D}$ & $157.4 \pm 11.8 \pm 2.2[57,59]$ & - \\
$f_{D_{s}}$ & $193.7 \pm 9.2 \pm 4.9[60]$ & - \\
& $193.0 \pm 11.3 \pm 4.9[61]$ & $\left.223 \pm 18[64]^{\mathrm{f}}\right]^{\mathrm{e}}$ \\
$f_{D^{*}}$ & $200.1 \pm 12.0 \pm 9.9[62]$ & $164 \pm 15[65]^{\mathrm{g}}$ \\
& - & $180 \pm 4 \pm 11[63]^{\mathrm{h}}$ \\
$f_{D_{s}^{*}}$ & - & $237 \pm 18[64]^{\mathrm{i}}$ \\
& - & $231 \pm 13[65]^{\mathrm{j}}$ \\
\hline Decay constant & - & Value used in this work \\
\hline$f_{\eta^{\prime}}$ & & $f_{\eta}$ \\
$f_{D_{s}}$ & & $193.7 \mathrm{MeV}$ \\
$f_{D^{*}}=f_{D_{s}^{*}}$ & & $f_{D}$ \\
\hline \hline
\end{tabular}

${ }^{\mathrm{a}}$ From $\Gamma\left(\rho \rightarrow e^{+} e^{-}\right)=4 \pi \alpha^{2} f_{\rho}^{2} / 3 m_{\rho} \quad$ and $\quad \Gamma\left(\tau \rightarrow \rho \nu_{\tau}\right)=$ $G^{2} \cos ^{2} \theta_{C} f_{\rho}^{2}\left(m_{\tau}^{2}-m_{\rho}^{2}\right)^{2}\left(2 m_{\rho}^{2}+m_{\tau}^{2}\right) / 8 \pi m_{\tau}^{3}$.

${ }^{\mathrm{b}}$ From $\quad \Gamma\left(\tau \rightarrow K^{*} \nu_{\tau}\right)=G^{2} \sin ^{2} \theta_{C} f_{K^{*}}^{2}\left(m_{\tau}^{2}-m_{K^{*}}^{2}\right)^{2} \times$ $\left(2 m_{K^{*}}^{2}+m_{\tau}^{2}\right) / 8 \pi m_{\tau}^{3}$.

${ }^{\mathrm{c}}$ From $\Gamma\left(\omega \rightarrow e^{+} e^{-}\right)=4 \pi \alpha^{2} f_{\omega}^{2} / 27 m_{\omega}$.

${ }^{\mathrm{d}}$ From $\Gamma\left(\phi \rightarrow e^{+} e^{-}\right)=8 \pi \alpha^{2} f_{\phi}^{2} / 27 m_{\phi}$.

${ }^{\mathrm{e}}$ Quenched LQCD.

${ }_{\mathrm{f}}^{\mathrm{f}}$ Relativistic constituent quark model.

${ }^{\mathrm{g}}$ Nonrelativistic constituent quark model.

${ }^{\mathrm{h}}$ Quenched LQCD.

${ }^{\mathrm{i}}$ Relativistic constituent quark model.

${ }^{\mathrm{j}}$ Nonrelativistic constituent quark model.
$(0,1 / 2,0,1)$, then the same value will be used in all other sectors.

The mass and widths of the dynamically generated resonances in each $I J S C$ sector are determined from the positions of the poles, $z_{R}$, in the second Riemann sheet of the corresponding scattering amplitudes, namely $M_{R}=$ $\operatorname{Re}\left(z_{R}\right)$ and $\Gamma_{R}=-2 \operatorname{Im}\left(z_{R}\right)$. The coupling constants of each resonance to the various baryon-meson states are obtained from the residues by fitting the amplitudes to the expression

$$
T_{i j}^{I J S C}(z)=\frac{g_{i} e^{i \phi_{i}} g_{j} e^{i \phi_{j}}}{\left(z-z_{R}\right)},
$$

for complex energy values $z$ close to the pole, where the complex couplings are written in terms of the absolute value, $g_{k}$, and phase, $\phi_{k}$. These dimensionless couplings determine the corresponding decaying branching ratios to the open baryon-meson channels. Since the dynamically generated states may couple differently to their baryonmeson components, we will examine the $i j$-channel independent quantity

$$
\tilde{T}^{I J S C}(z) \equiv \max _{j} \sum_{i}\left|T_{i j}^{I J S C}(z)\right|,
$$

which allows us to identify all the resonances within a given sector at once.

\section{SU(8) RESULTS}

In this work we have focused on the nonstrange $(S=0)$, singly charmed $(C=1)$ baryon resonances. The experimental status in this sector is summarized in Table III. In the following tables and figures, we collect results of resonances up to $3.5 \mathrm{GeV}$, which is basically the energy of the heaviest channels used in the present approach. Note, however, that the position and width of dynamical states generated $\sim 500 \mathrm{MeV}$ beyond the lowest threshold for each IJSC sector must be taken with caution. In reality, they will be strongly influenced by other ingredients not included in the present model, such as the coupling to three-body meson-meson-baryon states or interactions of

TABLE III. Summary of experimental data for baryon resonances with charm 1, zero strangeness and negative (or unknown) parity as compiled in Ref. [57]. The $\Lambda_{c}$ and $\Sigma_{c}$ ground states are omitted.

\begin{tabular}{lcccc}
\hline \hline Resonance & $I\left(J^{P}\right)$ & Status & Mass $(\mathrm{MeV})$ & $\Gamma(\mathrm{MeV})$ \\
\hline$\Lambda_{c}(2595)$ & $0\left(1 / 2^{-}\right)$ & $* * *$ & $2595.4 \pm 0.6$ & $3.6+2.0-1.3$ \\
$\Lambda_{c}(2625)$ & $0\left(3 / 2^{-}\right)$ & $* * *$ & $2628.1 \pm 0.6$ & $<1.9$ \\
$\Lambda_{c}(2765)$ & $?\left(?^{?}\right)$ & $*$ & $2766.6 \pm 2.4$ & 50 \\
or $\Sigma_{c}(2765)$ & & & & \\
$\Lambda_{c}(2880)$ & $0\left(5 / 2^{+}\right)$ & $* * *$ & $2881.9 \pm 0.5$ & $5.8 \pm 1.9$ \\
$\Lambda_{c}(2940)$ & $0\left(?^{?}\right)$ & $* * *$ & $2939.8 \pm 1.6$ & $18 \pm 8$ \\
$\Sigma_{c}(2800)^{++}$ & $1\left(?^{?}\right)$ & $* * *$ & $2801+4-6$ & $75+22-17$ \\
$\Sigma_{c}(2800)^{+}$ & $1\left(?^{?}\right)$ & $* * *$ & $2792+14-5$ & $62+60-40$ \\
$\Sigma_{c}(2800)^{0}$ & $1\left(?^{?}\right)$ & $* * *$ & $2802+4-7$ & $61+28-18$ \\
\hline \hline
\end{tabular}


higher angular momentum. This observation applies especially to those states higher than $3 \mathrm{GeV}$ that couple strongly to low-lying baryon-meson channels.

$\begin{array}{cccc}\Sigma_{c} \pi & N D & \Lambda_{c} \eta & N D^{*} \\ 2591.6 & 2806.15 & 2833.91 & 2947.27 \\ \Lambda D_{s}^{*} & \Sigma_{c} \rho & \Lambda_{c} \eta^{\prime} & \Sigma_{c}^{*} \rho \\ 3227.98 & 3229.05 & 3244.24 & 3293.46\end{array}$

where the second line gives the channel thresholds in $\mathrm{MeV}$.

In Fig. 1 we show our results for $\tilde{T}$ as a function of the CM energy $\sqrt{s}$, where we have used the experimentally known meson decay constants and have chosen $f_{D_{s}}=$ 193.7 MeV. For $\eta^{\prime}, D^{*}$, and $D_{s}^{*}$, we have taken $f_{\eta^{\prime}}=f_{\eta}$ and $f_{D^{*}}=f_{D_{s}^{*}}=f_{D}$ (see Table II). The dotted line shows that a narrow resonance is produced very close to the position of the nominal $\Lambda_{c}(2595)$, which is denoted by the starred line in the figure. By slightly changing the value of the subtraction point in the renormalization scheme, viz. setting the scaling factor $\alpha$ to 0.9698 [see Eq. (17)], we can reproduce the position of the $\Lambda_{c}(2595)$.

There are several other narrow resonances and bumps in Fig. 1, which can be better disentangled by investigating the behavior of the function $\tilde{T}$ and $T$ in the complex plane. We obtain the poles of $T$ in the second Riemann sheet and determine the coupling constants to the various baryonmeson channels through the residues of the corresponding amplitudes, as indicated in Eq. (18). The positions of the poles of $T$ are visualized in Fig. 2 as peaks of $\tilde{T}$, while the values of the mass and width of the corresponding resonances, together with the values of the couplings to the

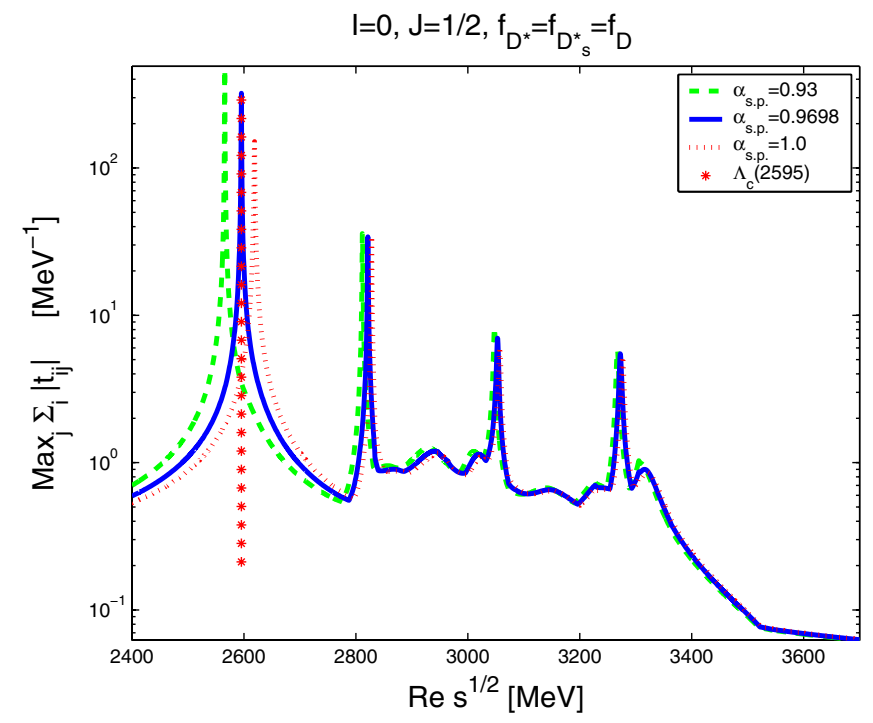

FIG. 1 (color online). The function $\tilde{T} \equiv \max _{j} \sum_{i}\left|T_{i j}\right|$ in the $I=0, J=1 / 2$ sector calculated along the scattering line within our broken SU(8) model, for different values of the subtraction point. The stars denote the nominal position of the $\Lambda_{c}(2595)$ resonance.

$$
\text { A. } I=0, J=1 / 2
$$

In this sector the following 16 channels are involved:

$\begin{array}{cccc}\Xi_{c} K & \Lambda_{c} \omega & \Xi_{c}^{\prime} K & \Lambda D_{s} \\ 2965.12 & 3069.03 & 3072.52 & 3084.18 \\ \Lambda_{c} \phi & \Xi_{c} K^{*} & \Xi_{c}^{\prime} K^{*} & \Xi_{c}^{*} K^{*} \\ 3305.92 & 3363.33 & 3470.73 & 3540.23\end{array}$

baryon-meson components to which they couple most strongly, are collected in Table IV.

The width of the $\Lambda_{c}$ (2595) resonance turns out to be small: $0.58 \mathrm{MeV}$, smaller than the experimental width, $\Gamma=$ $3.6_{-1.3}^{+2.0}$. However, we have not included here the threebody decay channel $\Lambda_{c} \pi \pi$, which already represents almost one-third of the decay events [57]. The narrowness of this resonance is due to the fact that it lies only a few $\mathrm{MeV}$ above its unique two-body decaying channel $\Sigma_{c} \pi$ to which it actually couples very weakly, as indicated by the low value of the corresponding coupling constant in Table IV.

Here, a remarkable feature is that there is a second resonance very close to $\Lambda_{c}(2595)$. This is precisely the same pattern found in the charmless $I=0, S=-1$ sector for the $\Lambda(1405)$ [22].

It is illustrative to compare the coupled-channel results with those from an uncoupled calculation as shown in Table V. In the lower energy region we find poles at $2622 \mathrm{MeV}, 2687 \mathrm{MeV}$, and $2775 \mathrm{MeV}$, which are, respectively, bound $\Sigma_{c} \pi, N D^{*}$, and $N D$ states. Note that the bound states in $N D$ and $N D^{*}$ channels are quite close. This emphasizes once more the importance of including

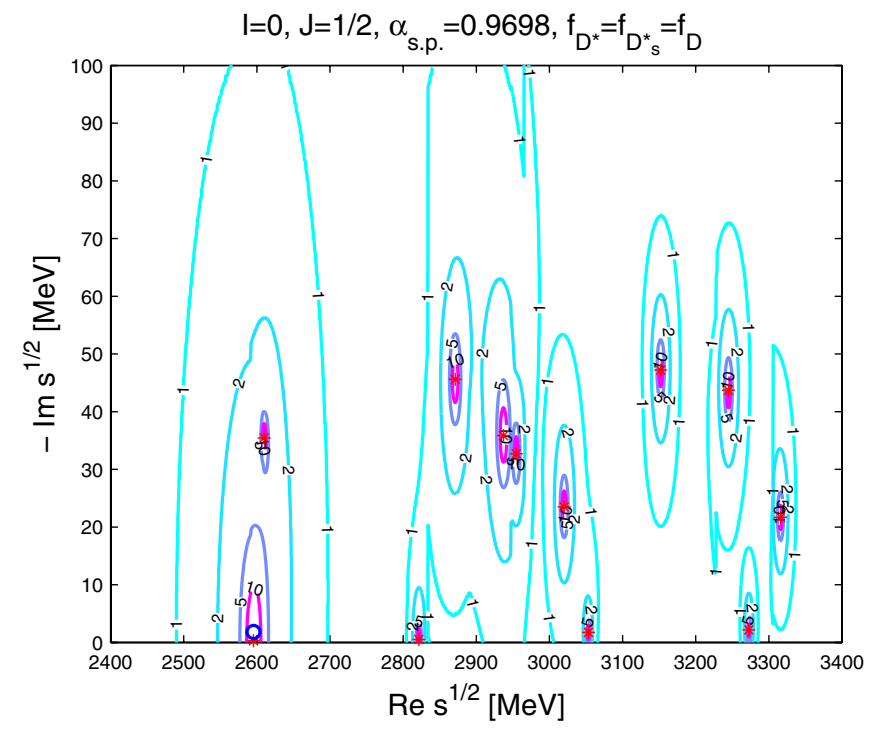

FIG. 2 (color online). The function $\tilde{T} \equiv \max _{j} \sum_{i}\left|T_{i j}\right|$ in the $I=0, J=1 / 2$ sector, calculated within the present broken SU (8) model with $\alpha=0.9698$. Stars show the positions of the resonances obtained, while open cycles denote those of the experimentally measured resonances, the $\Lambda_{c}(2595)$ in this figure. 
TABLE IV. Mass (MeV), width (MeV) and couplings of resonances with $S=0$ and $C=1$, generated in the $I=0, J=1 / 2$ sector using our broken SU(8) model. See text for more details. The third column quotes only the values of the most important couplings, and those shown in bold correspond to channels open for decay.

\begin{tabular}{|c|c|c|}
\hline $\begin{array}{l}I=0, J=1 / 2 \\
M_{R}\end{array}$ & $\Gamma_{R}$ & Couplings to main channels \\
\hline 2595.4 & 0.58 & $\boldsymbol{g}_{\Sigma_{c} \pi}=\mathbf{0 . 3 6}, g_{N D}=3.69, g_{N D^{*}}=5.70, g_{\Lambda D_{s}}=1.42, g_{\Lambda D_{s}^{*}}=2.94$ \\
\hline 2610.0 & 70.9 & $g_{\Sigma_{c} \pi}=\mathbf{2 . 2 5}, g_{N D}=1.47, g_{N D^{*}}=1.81, g_{\Sigma_{c} \rho}=1.22$ \\
\hline 2821.5 & 1.0 & $\boldsymbol{g}_{N D}=\mathbf{0 . 3 2}, g_{\Lambda_{c} \eta}=1.2, g_{\Xi_{c} K}=1.79, g_{\Lambda D_{s}^{*}}=1.11, g_{\Sigma_{c} \rho}=1.23, g_{\Sigma_{c}^{*} \rho}=1.15$ \\
\hline 2871.2 & 91.2 & $\boldsymbol{g}_{N D}=\mathbf{2 . 0}, g_{\Lambda D_{s}}=1.15, g_{N D^{*}}=2.15, g_{\Lambda D_{s}^{*}}=1.92, g_{\Lambda_{c} \omega}=1.01, g_{\Sigma_{c} \rho}=2.56, g_{\Sigma_{c}^{*} \rho}=0.9$ \\
\hline 2937.2 & 71.7 & $g_{\Lambda_{c} \eta}=1.34, g_{\Lambda D_{s}}=1.4, g_{N D^{*}}=1.51, g_{\Lambda D_{s}^{*}}=3.41, g_{\Sigma_{c}^{*} \rho}=2.23$ \\
\hline 2954.7 & 65.4 & $g_{\Sigma_{c} \pi}=1.02, g_{\Xi_{c}^{\prime} K}=1.2, g_{\Lambda D_{s}}=0.85, g_{\Lambda_{c} \omega}=2.46, g_{\Sigma_{c} \rho}=1.16, g_{\Sigma_{c}^{*} \rho}=0.91$ \\
\hline 3020.1 & 47.0 & $g_{\Xi_{c} K}=1.13, g_{\Lambda D_{s}}=1.07, g_{\Lambda D_{s}^{*}}=1.46, g_{\Sigma_{c} \rho}=1.51, g_{\Sigma_{c}^{*} \rho}=2.49, g_{\Xi_{c}^{*} K^{*}}=0.95$ \\
\hline 3053.3 & 3.49 & $\boldsymbol{g}_{\Lambda_{c} \omega}=\mathbf{0 . 1 1}, g_{\Lambda D_{s}}=1.43, g_{\Xi_{c}^{\prime} K}=1.49, g_{\Lambda_{c} \phi}=1.27, g_{\Sigma_{c}^{*} \rho}=1.02$ \\
\hline 3152.1 & 94.4 & $g_{\Lambda D_{s}}=1.74, g_{\Lambda D_{s}^{*}}=2.02, g_{\Lambda_{c} \phi}=2.17, g_{\Xi_{c}^{\prime} K^{*}}=0.98, g_{\Xi_{c} K^{*}}=1.38, g_{\Xi_{c}^{*} K^{*}}=1.16$ \\
\hline 3244.7 & 87.4 & $\boldsymbol{g}_{\boldsymbol{\Lambda} D_{s}}=\mathbf{0 . 7 2}, \boldsymbol{g}_{\boldsymbol{\Lambda} D_{s}^{*}}=\mathbf{0 . 7 4}, \boldsymbol{g}_{\Xi_{c} K}=\mathbf{0 . 6 8}, g_{\Xi_{c}^{\prime} K^{*}}=2.32, g_{\Xi_{c}^{*} K^{*}}=2.63$ \\
\hline 3272.6 & 4.31 & $g_{\Xi_{c}^{\prime} K}=\mathbf{0 . 1 7}, \boldsymbol{g}_{\Sigma_{c} \boldsymbol{\rho}}=\mathbf{0 . 1 7}, \boldsymbol{g}_{\boldsymbol{\Lambda} D_{s}}=\mathbf{0 . 1 5}, g_{\Lambda_{c} \phi}=1.37, g_{\Xi_{c} K^{*}}=2.26$ \\
\hline 3315.9 & 43.4 & $g_{\Sigma_{c} \boldsymbol{\rho}}=\mathbf{0 . 4 7}, \boldsymbol{g}_{\boldsymbol{\Lambda} D_{s}}=\mathbf{0 . 6 2}, \boldsymbol{g}_{\boldsymbol{\Lambda}_{c} \boldsymbol{\phi}}=\mathbf{0 . 6 6}, g_{\Xi_{c} K^{*}}=1.2, g_{\Xi_{c}^{\prime} K^{*}}=1.91, g_{\Xi_{c}^{*} K^{*}}=2.38$ \\
\hline
\end{tabular}

the vector mesons in the description. The effect of the channel coupling may be easily traced by taking into account the largest coefficients in Table XV, together with the channel threshold energies. We conclude that the resonance at $2595.4 \mathrm{MeV}$ appears to be basically the remnant of the $N D^{*}$ bound state found at $2687 \mathrm{MeV}$ in the uncoupled calculation, but with the important additional binding effects from the $N D$ channel, to which it couples very strongly, along with some moderate modifications induced by coupling to $\Lambda D_{s}$ and $\Lambda D_{s}^{*}$ the latter lying more than $400 \mathrm{MeV}$ away. As emphasized in the next section, we observe here that the nature of this resonance is substantially different from that found in Refs. [50,52], where it is identified as a $N D$ bound state. A wider resonance at $2610 \mathrm{MeV}$ originates from the $\Sigma_{c} \pi$ resonance found at $2622 \mathrm{MeV}$ in the uncoupled calculation, mildly modified by its coupling to $N D$ as well as to some channels involving vector mesons such as $N D^{*}$ and $\Sigma_{c} \rho$. We do not find a clear remnant of the uncoupled $N D$ state at $2775 \mathrm{MeV}$, apart from the already commented influence on the properties of the $\Lambda_{c}(2595)$ resonance.

A narrow resonance at $2822 \mathrm{MeV}$ originates mainly from the $\Xi_{c} K$ bound state at $2948 \mathrm{MeV}$, which is substantially influenced by the coupling to the $\Lambda_{c} \eta$ channel as well as to a few other channels $\left(\Lambda D_{s}^{*}, \Sigma_{c} \rho\right.$ and $\left.\Sigma_{c}^{*} \rho\right)$ involving vector mesons. The two other narrow resonances found in this sector, at $3053 \mathrm{MeV}$ and $3273 \mathrm{MeV}$, can be traced back, respectively, to the $\Xi_{c}^{\prime} K$ bound state at $3043 \mathrm{MeV}$ and the $\Xi_{c} K^{*}$ bound state at $3293 \mathrm{MeV}$ of the uncoupled calculation. The resonance at $3053 \mathrm{MeV}$ shows

TABLE V. Positions of poles (in MeV) generated in the $J=1 / 2, I=0$ sector from uncoupled calculations in the present broken SU(8) model with $\alpha=0.9698$. An asterisk $*$ denotes resonances.

\begin{tabular}{|c|c|c|c|c|c|c|c|c|c|}
\hline Channel $\left(M_{\text {thres }}\right)$ & 2600 & 2700 & 2800 & 2900 & 3000 & 3100 & 3200 & 3300 & 3400 \\
\hline$\Sigma_{c} \pi(2592)$ & $2621.6^{*}$ & & & & & & & & \\
\hline$N D(2806)$ & & 2775.4 & & & & & & & \\
\hline$\Lambda_{c} \eta$ & & & & & & & & & \\
\hline$N D^{*}(2947)$ & 2687.3 & & & & & & & & \\
\hline$\Xi_{c} K(2965)$ & & & & 2947.7 & & & & & \\
\hline$\Lambda_{c} \omega(3069)$ & & & & & & & & & \\
\hline$\Xi_{c}^{\prime} K(3073)$ & & & & & 3042.8 & & & & \\
\hline$\Lambda D_{s}(3084)$ & & & & & & & & & \\
\hline$\Lambda D_{s}^{*}(3228)$ & & & & & 3057.3 & & & & \\
\hline$\Sigma_{c} \rho(3229)$ & & & & 2962.6 & & & & & \\
\hline$\Lambda_{c} \eta^{\prime}$ & & & & & & & & & \\
\hline$\Sigma_{c}^{*} \rho(3293)$ & & & & 2988.5 & & & & & \\
\hline$\Lambda_{c} \phi(3306)$ & & & & & & & & & \\
\hline$\Xi_{c} K^{*}(3363)$ & & & & & & & 3293.3 & & \\
\hline$\Xi_{c}^{\prime} K^{*}(3471)$ & & & & & & & & 3382.6 & \\
\hline$\Xi_{c}^{*} K^{*}(3540)$ & & & & & & & & & 3440.2 \\
\hline
\end{tabular}


also a strong coupling to a few other channels, especially to the neighboring $\Lambda D_{s}$ state.

Most of the remaining wider resonances show also a stronger coupling to a baryon-meson system for which the uncoupled calculation produces a bound state. They are simply wider because they also couple significantly to meson-baryon states lying below their mass. However, we identify two resonances that occur genuinely as a result of the coupled-channel formalism, since their main baryon-meson component $\left(\Lambda_{c} \omega\right.$ for the resonance at $2955 \mathrm{MeV}$ and $\Lambda_{c} \phi$ for the resonance at $3152 \mathrm{MeV}$ ) does not have a bound state in the uncoupled calculation.

In order to study possible variations of the result due to input parameters, we have also performed calculations by adopting the values for the unknown decay constants of the charmed vector mesons from a quenched lattice QCD, LQCD [63]: $f_{D^{*}}=165 \mathrm{MeV}, f_{D_{s}^{*}}=180 \mathrm{MeV}$. Then the $\Lambda_{c}(2595)$ is reproduced with a slightly smaller scaling factor $(\alpha=0.9558)$, but the qualitative features for the positions, widths and couplings of all the other resonances generated in this sector do not change drastically.

In comparing with data, we look at the possibility of identifying some of our narrow states with a resonance seen experimentally. Our predictions are limited by the fact that we have implemented neither the coupling to threebody states nor $p-, d$ - or higher-multipolarity interactions. In the present $I=0, J=1 / 2$ sector, we have already identified our first narrow state with the $\Lambda_{c}$ (2595) and have established it as being a quasibound $N D^{*}$ system. A resonance at $2880 \mathrm{MeV}$ with a width of $\Gamma \sim 6 \mathrm{MeV}$, decaying into $\Lambda_{c} \pi \pi$ states with some fraction of resonant decay through $\Sigma_{c} \pi$ states, was reported in [42]. One might identify it with our narrow resonance at $2822 \mathrm{MeV}$, especially because if this state were moved to the experimental energy of $2880 \mathrm{MeV}$, it would appear above the threshold of the $\Lambda_{c} \eta$ channel to which it couples significantly. However, the Belle Collaboration determined recently the spin of the $\Lambda_{c}(2880)$ to be $J=5 / 2$ from the $\Sigma_{c}(2455) \pi$ decay angular distribution, and the parity positive from agreement of the $\Gamma\left(\Sigma_{c}(2520) \pi\right) / \Gamma\left(\Sigma_{c}(2455) \pi\right)$ branching ratio with a prediction of HQS [69-72]. These assignments do not match the spin-parity $1 / 2^{-}$of our dynamically generated state.

There is yet another resonance in this sector, the $\Lambda_{c}$ (2940) of width $\Gamma \sim 18 \pm 8 \mathrm{MeV}$, which has been seen in the $D p$ invariant mass distributions, but its spin and parity have not been determined [46]. If it were a $J=$ $1 / 2^{-}$state, one might be tempted to identify it with one of our narrow states. However, none of the resonances obtained by our model having a width smaller than $50 \mathrm{MeV}$, except for the lowest-lying one already identified with the $\Lambda_{c}$ (2595), couples significantly to the $N D$ states from which the invariant mass of the $\Lambda_{c}(2940)$ is reconstructed. Our model does not give either any resonance in this energy region that couples preferentially to $N D^{*}$ states, the threshold of which is only a few $\mathrm{MeV}$ above the $\Lambda_{c}$ (2940) mass. Therefore, we do not expect the $\Lambda_{c}(2940)$ to be a molecular $N D^{*}$ system, as claimed in the literature [73]. We finally note that the $p D^{0}$ histogram shown in Ref. [46] is not incompatible with the existence of a very narrow state just above the $N D$ threshold, as the one at $2822 \mathrm{MeV}$ and width $1 \mathrm{MeV}$ found in the present work, as well as in the dynamical model of Ref. [50].

\section{B. $I=1, J=1 / 2$}

In the $I=1, J=1 / 2$ sector there are 22 channels involved:

\begin{tabular}{ccccccccccc}
$\Lambda_{c} \pi$ & $\Sigma_{c} \pi$ & $N D$ & $N D^{*}$ & $\Xi_{c} K$ & $\Sigma_{c} \eta$ & $\Lambda_{c} \rho$ & $\Xi_{c}^{\prime} K$ & $\Sigma D_{s}$ & $\Delta D^{*}$ & $\Sigma_{c} \rho$ \\
2424.5 & 2591.6 & 2806.15 & 2947.27 & 2965.12 & 3001.01 & 3061.95 & 3072.52 & 3161.65 & 3218.35 & 3229.05 \\
$\Sigma_{c} \omega$ & $\Sigma_{c}^{*} \rho$ & $\Sigma_{c}^{*} \omega$ & $\Sigma D_{s}^{*}$ & $\Xi_{c} K^{*}$ & $\Sigma_{c} \eta^{\prime}$ & $\Xi_{c}^{\prime} K^{*}$ & $\Sigma_{c} \phi$ & $\Sigma^{*} D_{s}^{*}$ & $\Sigma_{c}^{*} \phi$ & $\Xi_{c}^{*} K^{*}$. \\
3236.13 & 3293.46 & 3300.54 & 3305.45 & 3363.33 & 3411.34 & 3470.73 & 3473.02 & 3496.87 & 3537.43 & 3540.23 \\
\hline
\end{tabular}

Our results are presented in Fig. 3 and Table VI. First, we find a very narrow resonance at $2554 \mathrm{MeV}$, which is basically composed of the $\Delta D^{*}$ component (somewhat mixed with the $N D$ and $\Sigma^{*} D_{s}^{*}$ states). Hence, this resonance would be absent in models not including channels with a vector meson and a $3 / 2^{+}$baryon. Kinematically it can only decay to $\Lambda_{c} \pi$, but the corresponding coupling is very weak.

At higher energies and up to $3.4 \mathrm{GeV}$, our model generates many resonances in this sector, most of them having rather appreciable widths. Some of these resonances find their analogue within the states obtained by the pioneer work of Lutz and Kolomeitsev [49], where only the mesonbaryon channels composed by an octet Goldstone boson plus a member of either the antitriplet or the sextet of open charm ground-state baryons were considered. The resonances at $2612 \mathrm{MeV}, 2637 \mathrm{MeV}$, and $2974 \mathrm{MeV}$, would correspond to the states in Ref. [49] found, respectively, at $2800 \mathrm{MeV}$ (coupling mostly to $\Lambda_{c} \pi$ ), $2700 \mathrm{MeV}$ (coupling mostly to $\Sigma_{c} \pi$ ), and $2985 \mathrm{MeV}$ (coupling mostly to $\Sigma_{c} \eta$ ). The states obtained here appear at a lower mass and show a different width due to their coupling to many of the new baryon-meson components considered in this work.

From the experimental side, an isotriplet of excited charmed baryons, $\Sigma_{c}(2800)$ has been reported [43], decaying mainly to $\Lambda_{c}^{+} \pi^{-}, \Lambda_{c}^{+} \pi^{0}$ and $\Lambda_{c}^{+} \pi^{+}$pairs with a large width of around $60 \mathrm{MeV}$ (but with a $>50 \%$ error). This resonance has been tentatively assumed to decay to $\Lambda_{c} \pi$ in $d$ wave with its spin parity assumed as $J^{P}=3 / 2^{-}$relying on quark models, but angular distributions have not yet 
$\mathrm{I}=1, \mathrm{~J}=1 / 2, \alpha_{\text {s.p. }}=0.9698, \mathrm{f}_{\mathrm{D}^{*}}=f_{D^{*}}=f_{\mathrm{s}}$

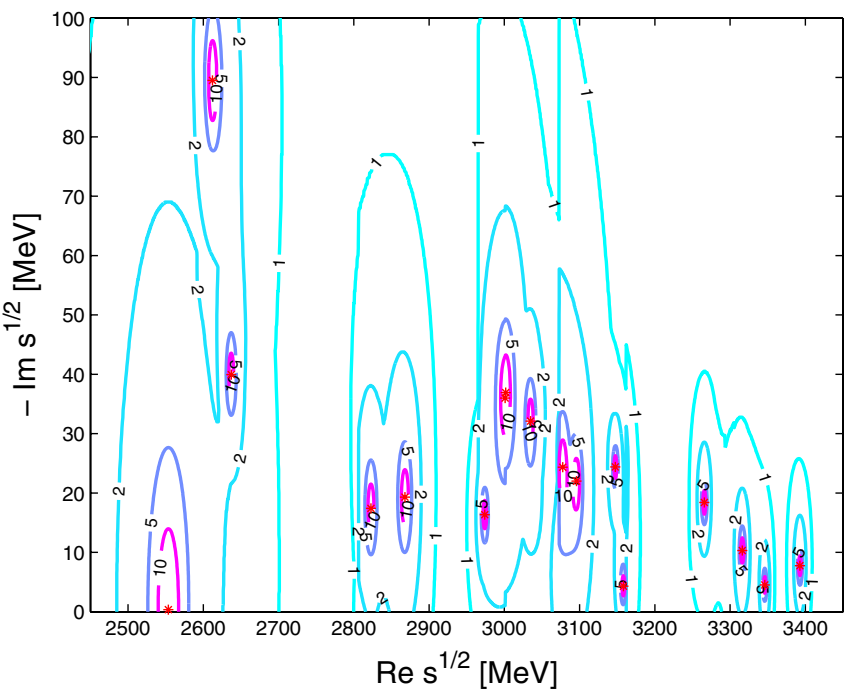

FIG. 3 (color online). The same as Fig. 2 for the $I=1, J=$ $1 / 2$ sector.

been measured. Of the predicted states discussed above having a moderate width, only the one at $3096 \mathrm{MeV}$ shows a sizable coupling to $\Lambda_{c} \pi$ states, but it lies $300 \mathrm{MeV}$ away of the experimental mass. Then what about identifying the predicted $3035 \mathrm{MeV}$ state as the $\Sigma_{c}(2800)$ ? Note first, that this $3035 \mathrm{MeV}$ state decays significantly to $\Sigma_{c} \pi$ pairs. Now if this state were to move to the experimental position, it would become somewhat narrower, especially because two of the possible decay channels, $\Sigma_{c} \eta$ and $N D^{*}$, would be closed. Its decay to $\Lambda_{c} \pi$ pairs, as observed experimentally, would require an implementation in the present model of an additional meson-baryon interaction in $d$-wave or/and $p$-wave coupling in the neglected $s$ - and

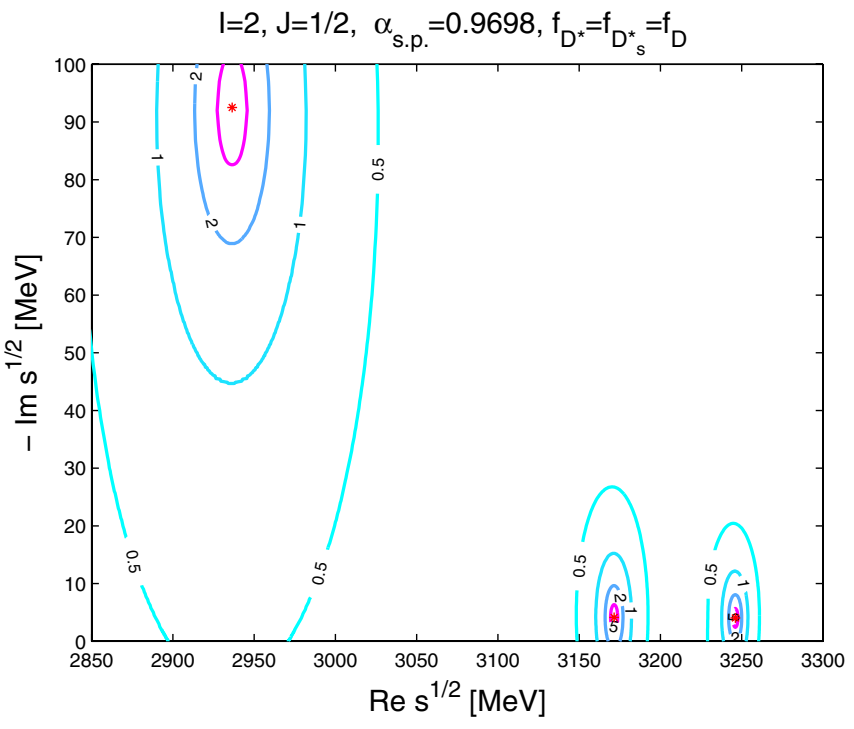

FIG. 4 (color online). The same as Fig. 2 for the $I=2, J=$ $1 / 2$ sector.

$u$-channel diagrams. However, according to the sizable coupling to $\Sigma_{c} \pi$ states, the identification of our state as $\Sigma_{c}(2800)$ would be ruled out if the latter were not observed in experiments looking at the $\Lambda_{c} \pi \pi$ systems. On inspecting the histograms of Ref. [42], one finds signals around $M\left(\Lambda_{c}^{+} \pi^{+} \pi^{-}\right)-M\left(\Lambda_{c}^{+}\right) \sim 500 \mathrm{MeV}$, corresponding to the mass of the $\Sigma_{c}(2800)$, although they are probably too feeble.

It seems unlikely that any of the three states with energies lying between 2800 and $3000 \mathrm{MeV}$ could qualify for the observed isotriplet, basically because they couple quite significantly to $N D$ states. Hence, if the parameters of our model would allow us to move one of these states below the $N D$ threshold, it would become significantly narrower

TABLE VI. The same as Table IV, for the $I=1, J=1 / 2$ sector.

\begin{tabular}{|c|c|c|}
\hline$\underline{M_{R}}$ & $\Gamma_{R}$ & $\begin{array}{l}\qquad I=1, J=1 / 2 \\
\text { Couplings to main channels }\end{array}$ \\
\hline 2553.6 & 0.67 & $\boldsymbol{g}_{\Lambda_{c} \pi}=\mathbf{0 . 1 5}, g_{N D}=2.28, g_{\Delta D^{*}}=6.74, g_{\Sigma^{*} D_{s}^{*}}=2.89$ \\
\hline 2612.2 & 179.0 & $g_{\Lambda_{c} \pi}=1.95, g_{N D^{*}}=3.78, g_{\Sigma_{c} \rho}=1.27, g_{\Sigma_{c}^{*} \rho}=1.4$ \\
\hline 2637.1 & 79.9 & $g_{\Sigma_{c} \pi}=1.98, g_{N D}=2.35, g_{N D^{*}}=1.69, g_{\Sigma^{*} D_{s}^{*}}=1.24$ \\
\hline 2822.8 & 34.8 & $\boldsymbol{g}_{N D}=1.55, g_{\Sigma D_{s}}=1.01, g_{\Xi_{c} K}=1.04, g_{N D^{*}}=1.41, g_{\Lambda_{c} \rho}=1.37, g_{\Delta D^{*}}=1.81, g_{\Sigma_{c}^{*} \rho}=2.27, g_{\Xi_{c}^{*} K^{*}}=0.94$ \\
\hline 2868.0 & 38.6 & $\boldsymbol{g}_{N D}=\mathbf{0 . 7 6}, g_{N D^{*}}=2.75, g_{\Sigma D_{s}^{*}}=1.05, g_{\Lambda_{c} \rho}=1.3, g_{\Sigma_{c} \rho}=1.54, g_{\Sigma_{c} \omega}=1.02, g_{\Delta D^{*}}=1.78$ \\
\hline 2974.0 & 37.2 & $\boldsymbol{g}_{N D}=\mathbf{0 . 7 2}, \boldsymbol{g}_{\Sigma_{c} \pi}=\mathbf{0 . 6 3}, \boldsymbol{g}_{N D^{*}}=\mathbf{0 . 6 3}, g_{\Sigma_{c} \eta}=1.67, g_{\Lambda_{c} \rho}=1.29, g_{\Sigma_{c} \omega}=1.13, g_{\Delta D^{*}}=1.21$ \\
\hline 3001.9 & 73.8 & $g_{\Xi_{c} K}=1.42, g_{\Sigma D_{s}}=1.68, g_{\Sigma D_{s}^{*}}=4.15, g_{\Sigma_{c} \rho}=1.42, g_{\Sigma_{c}^{*} \rho}=1.26$ \\
\hline 3034.9 & 64.2 & $g_{\Sigma_{c} \pi}=0.72, g_{\Sigma_{c} \eta}=0.67, g_{N D^{*}}=\mathbf{0 . 7 6}, g_{\Sigma_{D_{s}}}=1.16, g_{\Sigma_{c} \omega}=2.32, g_{\Sigma^{*} D_{s}^{*}}=2.44, g_{\Sigma_{c}^{*} \rho}=1.16, g_{\Sigma_{c}^{*} \omega}=0.87$ \\
\hline 3077.7 & 48.7 & $\boldsymbol{g}_{N D^{*}}=\mathbf{0 . 8 5}, g_{\Sigma_{c} \omega}=2.40, g_{\Sigma^{*} D_{s}^{*}}=3.20, g_{\Sigma_{c}^{*} \omega}=1.43$ \\
\hline 3095.9 & 44.0 & $\boldsymbol{g}_{\Lambda_{c} \pi}=\mathbf{0 . 3 9}, \boldsymbol{g}_{\Xi_{c}^{\prime} \boldsymbol{K}}=\mathbf{0 . 8 8}, \boldsymbol{g}_{N D^{*}}=\mathbf{0 . 4 5}, g_{\Sigma D_{s}}=1.34, g_{\Sigma_{c} \rho}=1.09, g_{\Sigma^{*} D_{s}^{*}}=2.80, g_{\Sigma_{c}^{*} \omega}=2.36$ \\
\hline 3147.5 & 48.8 & $\boldsymbol{g}_{\Lambda_{c} \boldsymbol{\rho}}=\mathbf{1 . 0 2}, g_{\Sigma_{c} \rho}=1.62, g_{\Sigma_{c} \omega}=0.94, g_{\Sigma_{c}^{*} \rho}=1.67, g_{\Sigma_{c}^{*} \omega}=1.57$ \\
\hline 3158.2 & 8.6 & $\boldsymbol{g}_{\Sigma_{c} \boldsymbol{\eta}}=\mathbf{0 . 3}, \boldsymbol{g}_{\boldsymbol{\Lambda}_{c} \boldsymbol{\rho}}=\mathbf{0 . 2 7}, g_{\Sigma D_{s}}=1.54, g_{\Sigma D_{s}^{*}}=1.51, g_{\Xi_{c} K^{*}}=1.53$ \\
\hline 3265.8 & 36.8 & $g_{\Sigma D_{s}}=0.69, g_{\Sigma D_{s}^{*}}=1.54, g_{\Sigma_{c} \phi}=1.32, g_{\Xi_{c}^{\prime} K^{*}}=1.49, g_{\Sigma_{c}^{*} \phi}=1.67, g_{\Xi_{c}^{*} K^{*}}=1.34$ \\
\hline 3316.2 & 20.6 & $\boldsymbol{g}_{\Xi_{c}^{\prime} K}=\mathbf{0 . 4 4}, g_{\Sigma_{c} \phi}=1.66, g_{\Xi_{c}^{\prime} K^{*}}=2.22, g_{\Sigma^{*} D_{s}^{*}}=1.80, g_{\Xi_{c}^{*} K^{*}}=1.01$ \\
\hline 3346.1 & 9.0 & $g_{\Sigma D_{s}}=\mathbf{0 . 4 5}, g_{\Sigma_{c} \phi}=1.81, g_{\Xi_{c} K^{*}}=1.12, g_{\Sigma_{c}^{*} \phi}=1.38, g_{\Xi_{c}^{*} K^{*}}=1.14$ \\
\hline 3392.8 & 15.5 & $\boldsymbol{g}_{\Sigma_{c}^{*} \boldsymbol{\rho}}=\mathbf{0 . 3 1}, g_{\Sigma_{c}^{*} \phi}=2.17, g_{\Xi_{c}^{*} K^{*}}=1.95$ \\
\hline
\end{tabular}


than the experimental width of $\sim 60 \mathrm{MeV}$. However, on inspecting the histogram of $I=1 D^{+} p$ pairs, shown in Ref. [46] to confirm the $\Lambda_{c}$ (2940) as being an isosinglet, one observes a clear enhancement around $2860 \mathrm{MeV}$ of width $\sim 10 \mathrm{MeV}$, which we could identify with one of the states we see decaying into $N D$ pairs. The most likely candidate is the state at $2974 \mathrm{MeV}$ because, if it was to be moved to $2860 \mathrm{MeV}$, its width would be reduced from $37 \mathrm{MeV}$ to a value of around $10 \mathrm{MeV}$ due to the closing of two out of three decaying channels.

Above $3 \mathrm{GeV}$ we find a few states that couple strongly to some vector meson-baryon channels. We thus expect them to be absent in the SU(4) models [50,52]. It may be of interest to note that the relatively narrow resonances at 3158 and $3346 \mathrm{MeV}$ could be seen from the invariant mass of $\Sigma_{c} \eta$ and $\Sigma D_{s}$ states, respectively.

$$
\text { C. } I=2, J=1 / 2
$$

In the $I=2, J=1 / 2$ sector there are four channels involved:

$$
\begin{array}{cccc}
\Sigma_{c} \pi & \Delta D^{*} & \Sigma_{c} \rho & \Sigma_{c}^{*} \rho \\
2591.6 & 3218.35 & 3229.05 & 3293.46
\end{array} .
$$

As seen from the results shown in Fig. 4 and Table VII, our model generates two narrow structures, at $3172 \mathrm{MeV}$ and $3246 \mathrm{MeV}$, which qualify, respectively, as $\Sigma_{c} \rho$ and $\Sigma_{c}^{*} \rho$ quasibound states.

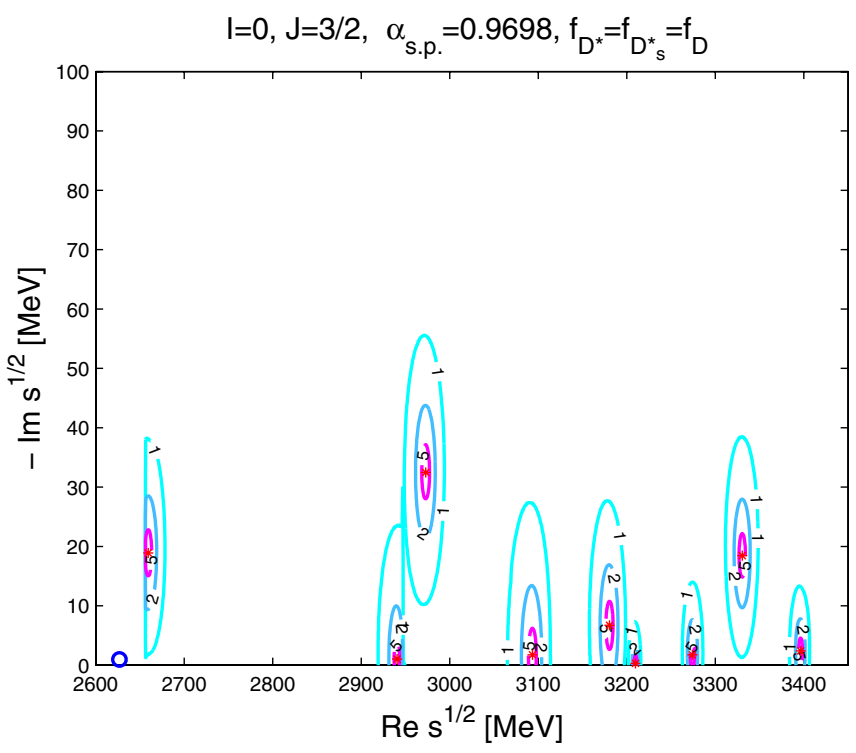

FIG. 5 (color online). The same as Fig. 2 for the $I=0, J=$ $3 / 2$ sector. Open cycles denote the positions of the experimentally measured resonances, $\Lambda_{c}(2625)$ in this case.

$$
\text { D. } I=\mathbf{0}, J=3 / 2
$$

Of various $J=3 / 2$ resonance candidates, we first consider those in the $I=0$ sector, with 11 coupled channels:

$$
\begin{array}{ccccccccccc}
\sum_{c}^{*} \pi & N D^{*} & \Lambda_{c} \omega & \Xi_{c}^{*} K & \Lambda D_{s}^{*} & \sum_{c} \rho & \sum_{c}^{*} \rho & \Lambda_{c} \phi & \Xi_{c} K^{*} & \Xi_{c}^{\prime} K^{*} & \Xi_{c}^{*} K^{*} \\
2656.01 & 2947.27 & 3069.03 & 3142.02 & 3227.98 & 3229.05 & 3293.46 & 3305.92 & 3363.33 & 3470.73 & 3540.23
\end{array} .
$$

The results displayed in Fig. 5 and Table VIII show that, in this sector, our model generates nine resonances, four of which have a width smaller than $5 \mathrm{MeV}$.

Experimentally, there is a three star $\Lambda_{c}(2625)$ resonance with $J^{P}=3 / 2^{-}$, which has a very narrow width, $\Gamma<$ $1.9 \mathrm{MeV}$, and decays mostly to $\Lambda_{c} \pi \pi$ [57]. It has been identified as the counterpart of the $\Lambda(1520)$ which, in dynamical models, shows a strong coupling to $\Sigma^{*} \pi$ [29$31,38,39]$. In the present $S=0, C=1$ sector, we indeed find a $I=0, J=3 / 2 d$-wave $\Lambda_{c}$ resonance at $2660 \mathrm{MeV}$ with its width $\Gamma=38 \mathrm{MeV}$, which couples very strongly to the $\Sigma_{c}^{*} \pi$ channel. We may therefore identify this resonance as a strong candidate for the $d$-wave $\Lambda_{c}(2625)$. A small change in the subtraction point $\mu^{I J S C}$ could easily move the resonance down by $40 \mathrm{MeV}$ to the nominal position. By so doing it should also be possible to reduce the width considerably, hence closer to the experimental value because the resonance position would get below the threshold of the channel $\Sigma_{c}^{*} \pi$ to which this resonance couples strongly. We note that a similar resonance was found at $2660 \mathrm{MeV}$ in the SU(4) model of Ref. [51]. The novel feature in the recent approach is that the resonance in question here has non-negligible baryon-vector-meson components such as $N D^{*}$ and $\Sigma_{c}^{*} \rho$. Other narrow resonances observed in this channel are a state at $3094 \mathrm{MeV}$ coupling strongly to $\Xi_{c}^{*} K$, as in Ref. [51], but also to the vector-meson channel $\Lambda D_{s}^{*}$ and three narrow resonances, which couple mostly to channels with vector-meson com-

TABLE VII. The same as Table IV, but for the $I=2, J=1 / 2$ sector.

\begin{tabular}{lcc}
\hline \hline & $I=2, J=1 / 2$ \\
$M_{R}$ & $\Gamma_{R}$ & Couplings to all channels \\
\hline 2936.5 & 185.0 & $\boldsymbol{g}_{\boldsymbol{\Sigma}_{c} \boldsymbol{\pi}}=\mathbf{1 . 6 2}, g_{\Delta D^{*}}=5.37, g_{\Sigma_{c} \rho}=1.12, g_{\Sigma_{c}^{*} \rho}=0.66$ \\
3171.5 & 8.26 & $\boldsymbol{g}_{\boldsymbol{\Sigma}_{c} \boldsymbol{\pi}}=\mathbf{0 . 3 3}, g_{\Delta D^{*}}=1.61, g_{\Sigma_{c} \rho}=2.26, g_{\Sigma_{c}^{*} \rho}=0.76$ \\
3246.1 & 8.09 & $\boldsymbol{g}_{\boldsymbol{\Sigma}_{c} \boldsymbol{\pi}}=\mathbf{0 . 1 8}, \boldsymbol{g}_{\Delta D^{*}}=\mathbf{0 . 3 3}, \boldsymbol{g}_{\boldsymbol{\Sigma}_{c} \boldsymbol{\rho}}=\mathbf{0 . 4 7 ,} g_{\Sigma_{c}^{*} \rho}=2.39$ \\
\hline \hline
\end{tabular}


TABLE VIII. The same as Table IV, for the $I=0, J=3 / 2$ sector.

\begin{tabular}{|c|c|c|}
\hline \multicolumn{3}{|r|}{$I=0, J=3 / 2$} \\
\hline$M_{R}$ & $\Gamma_{R}$ & Couplings to main channels \\
\hline 2659.5 & 37.8 & $g_{\Sigma_{c}^{*} \pi}=\mathbf{2 . 2 3}, g_{N D^{*}}=2.11, g_{\Sigma_{c}^{*} \rho}=1.34$ \\
\hline 2940.5 & 2.06 & $g_{\Sigma_{c}^{*} \pi}=\mathbf{0 . 2 1}, g_{N D^{*}}=2.21, g_{\Sigma_{c}^{*} \rho}=1.40$ \\
\hline 2972.8 & 64.9 & $\boldsymbol{g}_{\Sigma_{c}^{*} \pi}=\mathbf{1 . 0 5}, g_{\Lambda_{c} \omega}=2.42, g_{\Xi_{c}^{*} K}=0.95, g_{\Sigma_{c}^{*} \rho}=1.28$ \\
\hline 3093.5 & 3.48 & $\boldsymbol{g}_{\boldsymbol{\Lambda}_{c} \boldsymbol{\omega}}=\mathbf{0 . 2 9}, \boldsymbol{g}_{N D^{*}}=\mathbf{0 . 2 8}, g_{\Lambda D_{s}^{*}}=2.89, g_{\Xi_{c}^{*} K}=1.88$ \\
\hline 3180.5 & 13.4 & $g_{\Xi_{c}^{*} K}=\mathbf{0 . 6 9}, g_{\Lambda D_{s}^{*}}=2.49, g_{\Lambda_{c} \phi}=1.83, g_{\Xi_{c} K^{*}}=1.00, g_{\Xi_{c}^{*} K^{*}}=0.79$ \\
\hline 3209.8 & 0.6 & $\boldsymbol{g}_{N D^{*}}=\mathbf{0 . 0 8}, \boldsymbol{g}_{\Xi_{c}^{*} \boldsymbol{K}}=\mathbf{0 . 1 0}, g_{\Sigma_{c} \rho}=1.81, g_{\Sigma_{c}^{*} \rho}=1.09$ \\
\hline 3274.1 & 3.48 & $\boldsymbol{g}_{\boldsymbol{\Lambda} D_{s}^{*}}=\mathbf{0 . 1 9}, \boldsymbol{g}_{\Xi_{c}^{*} K}=\mathbf{0 . 2}, g_{\Lambda_{c} \phi}=1.30, g_{\Xi_{c} K^{*}}=2.31$ \\
\hline 3330.4 & 36.9 & $\boldsymbol{g}_{\boldsymbol{\Lambda} D_{s}^{*}}=\mathbf{0 . 5 0}, \boldsymbol{g}_{\boldsymbol{\Lambda}_{c} \boldsymbol{\phi}}=\mathbf{0 . 6 8}, \boldsymbol{g}_{\Sigma_{c}^{*} \boldsymbol{\rho}}=\mathbf{0 . 5 7}, g_{\Xi_{c} K^{*}}=0.85, g_{\Xi_{c}^{\prime} K^{*}}=1.85, g_{\Xi_{c}^{*} K^{*}}=2.34$ \\
\hline 3396.3 & 4.8 & $\boldsymbol{g}_{\boldsymbol{\Lambda} D_{s}^{*}}=\mathbf{0 . 1 7}, \boldsymbol{g}_{\Lambda_{c} \boldsymbol{\phi}}=\mathbf{0 . 2 2}, \boldsymbol{g}_{\Sigma_{c}^{*} \boldsymbol{\rho}}=\mathbf{0 . 1 5}, g_{\Xi_{c}^{\prime} K^{*}}=2.17, g_{\Xi_{c}^{*} K^{*}}=1.86$ \\
\hline
\end{tabular}

ponents. Out of the resonances obtained in this sector around $3 \mathrm{GeV}$ and beyond, the one at $2941 \mathrm{MeV}$ could be a candidate for the $\Lambda_{c}(2940)$ of width $\sim 18 \mathrm{MeV}$ and unknown $J^{P}$, observed recently from the invariant mass of $D^{0} p$ pairs [46]. Its decay into $D^{0} p$ pairs would imply the additional implementation of $p$-wave interactions in our

$\begin{array}{ccccc}\Sigma_{c}^{*} \pi & N D^{*} & \Lambda_{c} \rho & \Sigma_{c}^{*} \eta & \Delta D \\ 2656.01 & 2947.27 & 3061.95 & 3065.42 & 3077.23 \\ \Sigma_{c}^{*} \omega & \Sigma D_{s}^{*} & \Sigma^{*} D_{s} & \Xi_{c} K^{*} & \Xi_{c}^{\prime} K^{*} \\ 3300.54 & 3305.45 & 3353.07 & 3363.33 & 3470.73\end{array}$

Our results are presented in Fig. 6 and Table IX. We obtain many resonances and they all couple strongly, without exception, to channels with vector mesons. A bound state at $2550 \mathrm{MeV}$, whose main baryon-meson components contain a charmed meson, lies below the threshold of any possible decay channel. It would develop a narrow decay

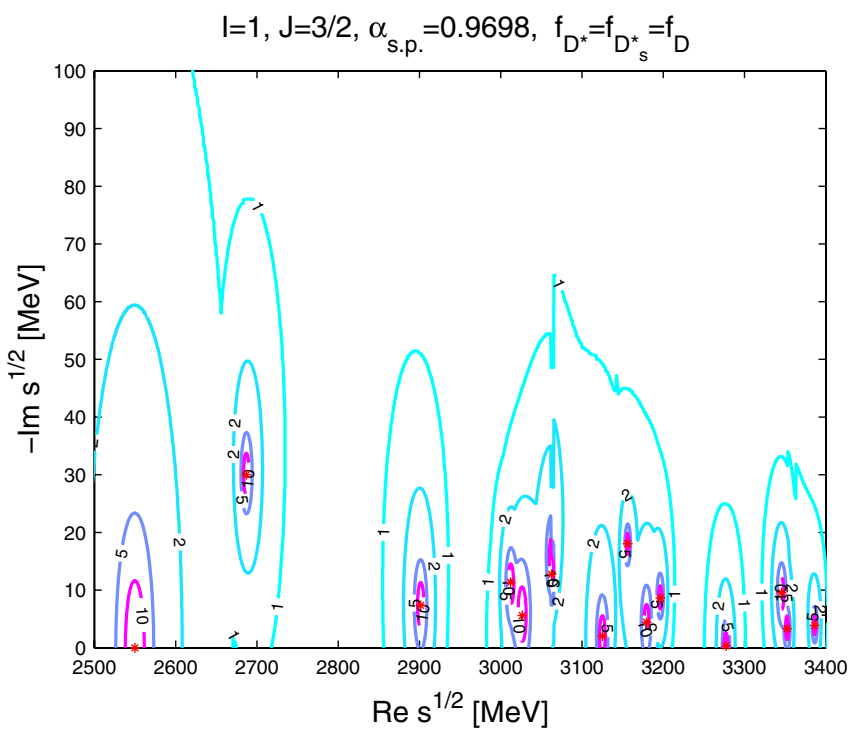

FIG. 6 (color online). The same as Fig. 2 for the $I=1, J=$ $3 / 2$ sector.

$\begin{array}{ccccc}\Xi_{c}^{*} K & \Delta D^{*} & \Sigma_{c} \rho & \Sigma_{c} \omega & \Sigma_{c}^{*} \rho \\ 3142.02 & 3218.35 & 3229.05 & 3236.13 & 3293.46 \\ \Sigma_{c} \phi & \Sigma_{c}^{*} \eta^{\prime} & \Sigma^{*} D_{s}^{*} & \Sigma_{c}^{*} \phi & \Xi_{c}^{*} K^{*} \\ 3473.02 & 3475.75 & 3496.87 & 3537.43 & 3540.23\end{array}$

model. The strong coupling of this resonance to $N D^{*}$ hints also for a substantial coupling into $p$-wave $N D$ states.

$$
\text { E. } I=1, J=3 / 2
$$

In the $I=1, J=3 / 2$ sector one finds 20 channels:

width to $\Lambda_{c} \phi \pi$ states if we incorporated $d$-wave interactions. This resonance is the charm sector counterpart of the hyperonic $\Sigma(1670)$ resonance, which has also been seen in chiral unitary models [29-31] coupling strongly to the $\Delta \bar{K}$ channel. Indeed, the charmed bound state found here couples strongly to the corresponding $\Delta D$ state in the charm sector, and even more strongly to its vector partner: $\Delta D^{*}$. This is precisely the reason for the large binding of this resonance, compared to that of its strange sector counterpart.

Since the observed $I=1$ charmed baryon resonances do not have spin nor parity assignments, most of the arguments discussed in the $I=1, J=1 / 2$ section, can also be made here. We might assign the resonance at $2687 \mathrm{MeV}$, decaying primarily to $\Sigma_{c}^{*} \pi$, to the bump at $2760 \mathrm{MeV}$ observed from $\Lambda_{c} \pi \pi$ states [42]. However, the corresponding width estimated by scaling our result $\Gamma_{2760}=$ $\left(q_{2760} / q_{2690}\right) \Gamma_{2690}$, would turn out to be around $120 \mathrm{MeV}$, much larger than the experimental value. Then, similarly to our discussion in the $I=1, J=1 / 2$ sector, the next state at $2901 \mathrm{MeV}$, decaying into $\Sigma_{c}^{*} \pi s$-wave pairs, could be a candidate for the $\Sigma_{c}(2800)$, seen in $\Lambda_{c} \pi$ pairs [43], if this resonance were also seen in $\Lambda_{c} \pi \pi$ states. The little enhancement in the $\Lambda_{c} \pi \pi$ histogram [42] around $M\left(\Lambda_{c}^{+} \pi^{+} \pi^{-}\right)-M\left(\Lambda_{c}^{+}\right) \sim 500 \mathrm{MeV}$, which is obviously distorted by the excitation of the lower mass resonance at $2760 \mathrm{MeV}$, might well correspond roughly to the mass of the $\Sigma_{c}(2800)$. 
TABLE IX. The same as Table IV, but for the $I=1, J=3 / 2$ sector.

\begin{tabular}{|c|c|c|}
\hline$M_{R}$ & $\Gamma_{R}$ & $\begin{array}{l}I=1, J=3 / 2 \\
\text { Couplings to main channels }\end{array}$ \\
\hline 2549.8 & 0.0 & $g_{N D^{*}}=2.52, g_{\Sigma D_{s}^{*}}=2.22, g_{\Delta D}=4.23, g_{\Sigma^{*} D_{s}}=1.48, g_{\Delta D^{*}}=5.28, g_{\Sigma^{*} D_{s}^{*}}=2.29$ \\
\hline 2686.9 & 60.0 & $g_{\Sigma_{c}^{*} \pi}=1.91, g_{N D^{*}}=2.68, g_{\Sigma D_{s}^{*}}=0.96, g_{\Lambda_{c} \rho}=1.02, g_{\Sigma^{*} D_{s}^{*}}=0.97$ \\
\hline 2901.6 & 14.7 & $g_{\Sigma_{c}^{*} \pi}=\mathbf{0 . 5 8}, g_{N D^{*}}=2.77, g_{\Lambda_{c} \rho}=1.52, g_{\Delta D}=1.26, g_{\Delta D^{*}}=1.05, g_{\Sigma_{c}^{*} \rho}=1.20$ \\
\hline 3012.1 & 22.7 & $\boldsymbol{g}_{N D^{*}}=\mathbf{0 . 9 0}, g_{\Sigma D_{s}^{*}}=2.05, g_{\Sigma_{c}^{*} \eta}=2.04$ \\
\hline 3026.5 & 11.2 & $\boldsymbol{g}_{N D^{*}}=\mathbf{0 . 7 2}, g_{\Sigma_{c} \omega}=2.10, g_{\Delta D}=2.40, g_{\Delta D^{*}}=2.83$ \\
\hline 3062.6 & 25.5 & $\boldsymbol{g}_{\Sigma_{c}^{*} \boldsymbol{\eta}}=\mathbf{0 . 6 1}, g_{\Sigma D_{s}^{*}}=1.67, g_{\Delta D^{*}}=1.56, g_{\Sigma^{*} D_{s}^{*}}=1.69, g_{\Sigma_{c}^{*} \omega}=1.97$ \\
\hline 3125.1 & 4.05 & $\boldsymbol{g}_{\Sigma_{c}^{*} \pi}=\mathbf{0 . 1 7}, \boldsymbol{g}_{\Sigma_{c}^{*} \boldsymbol{\eta}}=\mathbf{0 . 2 1}, g_{\Sigma D_{s}^{*}}=1.39, g_{\Sigma^{*} D_{s}}=1.67, g_{\Xi_{c}^{*} K}=1.33, g_{\Sigma^{*} D_{s}^{*}}=2.90, g_{\Sigma_{c}^{*} \omega}=0.93$ \\
\hline 3156.1 & 36.1 & $\boldsymbol{g}_{\Lambda_{c} \rho}=\mathbf{0 . 9}, g_{\Sigma D_{s}^{*}}=1.45, g_{\Sigma_{c} \rho}=1.50, g_{\Sigma_{c} \omega}=1.27, g_{\Sigma_{c}^{*} \rho}=1.41, g_{\Sigma_{c}^{*} \omega}=1.39$ \\
\hline 3179.5 & 8.89 & $g_{\Sigma_{c}^{*} \eta}=\mathbf{0 . 3 7}, g_{\Sigma_{D_{s}^{*}}^{*}}=2.65, g_{\Sigma_{c} \rho}=1.40, g_{\Xi_{c} K^{*}}=1.71, g_{\Sigma_{c}^{*} \omega}=0.85$ \\
\hline 3196.4 & 17.3 & $\boldsymbol{g}_{\Lambda_{c} \boldsymbol{\rho}}=\mathbf{0 . 5 6}, g_{\Sigma D_{s}^{*}}=0.96, g_{\Sigma_{c} \rho}=1.17, g_{\Sigma_{c} \omega}=0.87, g_{\Xi_{c} K^{*}}=0.87, g_{\Sigma_{c}^{*} \rho}=2.16, g_{\Sigma_{c}^{*} \omega}=0.92$ \\
\hline 3277.2 & 0.62 & $\boldsymbol{g}_{\Xi_{c}^{*} K}=\mathbf{0 . 1 1}, g_{\Sigma_{c} \phi}=0.91, g_{\Xi_{c}^{\prime} K^{*}}=1.68, g_{\Sigma^{*} D_{s}}=2.61, g_{\Sigma^{*} D_{s}^{*}}=2.52$ \\
\hline 3345.4 & 19.1 & $\boldsymbol{g}_{\Xi_{c}^{*} K}=\mathbf{0 . 5 4}, g_{\Xi_{c}^{\prime} K^{*}}=1.18, g_{\Sigma^{*} D_{s}^{*}}=1.07, g_{\sum_{c}^{*} \phi}=1.38, g_{\Xi_{c}^{*} K^{*}}=2.57$ \\
\hline 3352.6 & 6.65 & $\boldsymbol{g}_{\Sigma D_{s}^{*}}=\mathbf{0 . 4 0}, \boldsymbol{g}_{\Xi_{c}^{*} \boldsymbol{K}}=\mathbf{0 . 3 2}, g_{\Sigma_{c} \phi}=2.26, g_{\Xi_{c}^{\prime} K^{*}}=1.10, g_{\Xi_{c} K^{*}}=0.91, g_{\Sigma_{c}^{*} \phi}=1.72, g_{\Xi_{c}^{*} K^{*}}=0.99$ \\
\hline 3386.3 & 7.79 & $\boldsymbol{g}_{\Xi_{c} \boldsymbol{K}^{*}}=\mathbf{0 . 4 8}, g_{\Sigma_{c} \phi}=1.40, g_{\Xi_{c}^{\prime} K^{*}}=1.00, g_{\Sigma_{c}^{*} \phi}=2.20, g_{\Xi_{c}^{*} K^{*}}=1.18$ \\
\hline
\end{tabular}

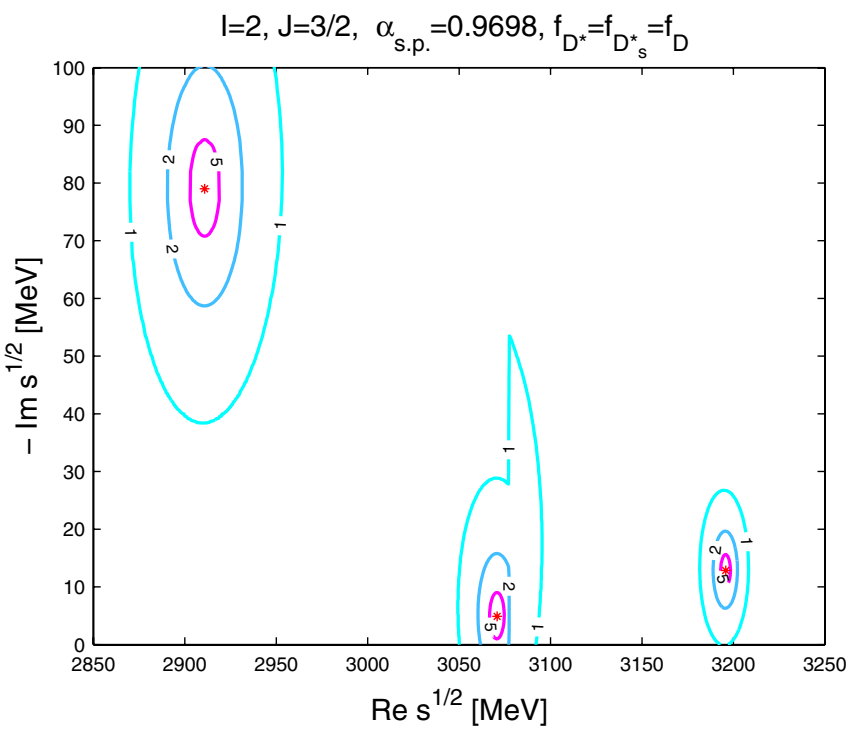

FIG. 7 (color online). The same as Fig. 2 for the $I=2, J=$ $3 / 2$ sector.

Our model predicts a few narrow resonances above $3 \mathrm{GeV}$. Of particular interest are those coupling strongly to $N D^{*}$, since they could be observed in $D \pi N$ invariant mass distributions and, if sufficiently narrow, these states should be reflected into the $D N$ invariant mass spectra. Although the discussion in Ref. [46] mentions explicitly the absence of signal in the $D^{*}(2010)^{+} p$ or $D^{*}(2007)^{0} p$ mass distributions, the invariant mass distribution of $D^{+} p$ pairs shows some structures which, unfortunately, are embedded into the statistical error fluctuations. Clearly, more data needs to be collected in order to see whether some of these structures acquire statistical significance to become new charmed isotriplet baryons.

$$
\text { F. } I=2, J=3 / 2
$$

Here, we present our results in the $I=2, J=3 / 2$ sector, where there are only five channels:

$$
\begin{array}{ccccc}
\Sigma_{c}^{*} \pi & \Delta D & \Delta D^{*} & \Sigma_{c} \rho & \Sigma_{c}^{*} \rho \\
2656.01 & 3077.23 & 3218.35 & 3229.05 & 3293.46
\end{array} \text {. }
$$

Our results, presented in Fig. 7 and Table X, indicate that the model generates a state at $2911 \mathrm{MeV}$, with mainly $N D$ and $N D^{*}$ components, which is very wide because it lies above the threshold of $\Sigma_{c}^{*} \pi$ states to which it also couples significantly. We also find a narrow resonance at $3071 \mathrm{MeV}$, which lies only a few $\mathrm{MeV}$ below the $\Delta D$ threshold and coupling more strongly to $\Delta D^{*}$ states, and a wider resonance at $3196 \mathrm{MeV}$, which qualifies as a $\Sigma_{c}^{*} \rho$ quasibound state.

$$
\text { G. } J=5 / 2(I=0,1 \text { and } 2)
$$

We finally present our results for the isospin sectors $I=$ 0,1 , and 2 with maximum value of $\operatorname{spin} J=5 / 2$. The

TABLE X. The same as Table IV, but for the $I=2, J=3 / 2$ sector.

\begin{tabular}{ccc}
\hline \hline \multicolumn{1}{c}{$I=2, J=3 / 2$} \\
Couplings to all channels
\end{tabular}


TABLE XI. The same as Table IV, but for the $I=0, J=5 / 2$ sector.

\begin{tabular}{lcc}
\hline \hline & \multicolumn{2}{c}{$I=0, J=5 / 2$} \\
$M_{R}$ & $\Gamma_{R}$ & Couplings to main channels \\
\hline 3258.0 & 0.0 & $g_{\Sigma_{c}^{*} \rho}=2.24$ \\
3440.2 & 0.0 & $g_{\Xi_{c}^{*} K^{*}}=2.87$ \\
\hline \hline
\end{tabular}

states building up the coupled-channel space in this $J=$ $5 / 2$ case are composed of a $3 / 2^{+}$baryon and a vector meson. Note that, as seen from the coefficients in Tables XXI, XXII, and XXIII, all these cases present the peculiarity that channels without strange quarks (as $\Sigma_{c}^{*} \rho$ or $\left.\Delta D^{*}\right)$ do not mix with channels having an associated $s \bar{s}$ pair (as $\Sigma_{c}^{*} \phi$ or $\Xi_{c}^{*} K^{*}$ ). Therefore, in the case $I=0, J=$ $5 / 2$, since the interaction is strongly attractive in the two channels involved, namely,

$$
\begin{array}{cc}
\sum_{c}^{*} \rho & \Xi_{c}^{*} K^{*} \\
3293.46 & 3540.23
\end{array}
$$

we obtain two states at $3258 \mathrm{MeV}$ and $3440 \mathrm{MeV}$ that are, respectively, bound $\Sigma_{c}^{*} \rho$ and $\Xi_{c}^{*} K^{*}$ systems, as seen in Table XI. Since we do not take into account three-particle channels, these resonances appear as stable bound states.

In the $I=1, J=5 / 2$ sector there are six channels involved:

$$
\Delta D^{*} \quad \Sigma_{c}^{*} \rho \quad \Sigma_{c}^{*} \omega \quad \Sigma^{*} D_{s}^{*} \quad \Sigma_{c}^{*} \phi \quad \Xi_{c}^{*} K^{*}
$$$$
3218.353293 .463300 .543496 .873537 .43 \quad 3540.23^{\circ}
$$

Table XII shows that we generate three bound states and a narrow resonance. The lower two states couple to the nonstrange baryon-meson channels, while the upper two belong to the associated strangeness sector.

Finally, we present in Table XIII the results for the $I=$ $2, J=5 / 2$ sector, which involves two channels:

$$
\begin{array}{cc}
\Delta D^{*} & \Sigma_{c}^{*} \rho \\
3218.35 & 3293.46
\end{array} .
$$

As seen from the coefficients in Table XXIII, the interaction is zero for $\Delta D^{*}$ states and repulsive for $\Sigma_{c}^{*} \rho$ states. In spite of this, our model generates a bound state at $3127 \mathrm{MeV}$, about $90 \mathrm{MeV}$ below the threshold of the $\Delta D^{*}$ channel and coupling strongly to it. This bound state is entirely due to the coupling between $\Delta D^{*}$ and $\Sigma_{c}^{*} \rho$

TABLE XII. The same as Table IV, but for the $I=1, J=5 / 2$ sector.

\begin{tabular}{llc}
\hline \hline & & $I=1, J=5 / 2$ \\
$M_{R}$ & $\Gamma_{R}$ & Couplings to main channels \\
\hline 3084.5 & 0.0 & $g_{\Delta D^{*}}=3.99, g_{\Sigma_{c}^{*} \rho}=0.98, g_{\Sigma_{c}^{*} \omega}=1.92$ \\
3233.8 & 0.17 & $g_{\Delta D^{*}}=\mathbf{0 . 1 4}, g_{\Sigma_{c}^{*} \rho}=2.14, g_{\Sigma_{c}^{*} \omega}=3.14$ \\
3276.7 & 0.0 & $g_{\Sigma^{*} D_{s}^{*}}=4.32, g_{\Sigma_{c}^{*} \phi}=1.17, g_{\Xi_{c}^{*} K^{*}}=1.95$ \\
3421.9 & 0.0 & $g_{\Sigma^{*} D_{s}^{*}}=0.15, g_{\Sigma_{c}^{*} \phi}=2.52, g_{\Xi_{c}^{*} K^{*}}=1.65$ \\
\hline \hline
\end{tabular}

TABLE XIII. The same as Table IV, but for the $I=2, J=5 / 2$ sector.

\begin{tabular}{lll}
\hline \hline & \multicolumn{2}{c}{$I=2, J=5 / 2$} \\
$M_{R}$ & $\Gamma_{R}$ & Couplings to all channels \\
\hline 3126.6 & 0.0 & $g_{\Delta D^{*}}=3.62, g_{\Sigma_{c}^{*} \rho}=2.0$ \\
\hline \hline
\end{tabular}

states, facilitated by the significant value of the mixing coefficient and by the vicinity of the channels involved, whose thresholds are only $75 \mathrm{MeV}$ apart. We have checked that the bound state disappears if the mixing coefficient is reduced to $2 / 3$ its actual value.

\section{COMPARISON WITH SU(4) MODELS}

In this section we would like to compare our results with those obtained within a standard SU(4) model denoted as TVME in the present work. We will follow the TVME approach, first developed in Ref. [50], but with modifications implemented in Ref. [52], which we describe briefly in the following. Firstly, the last term of the interaction kernel used in Ref. [50], namely,

$$
\begin{aligned}
V^{(I, S, C)}(\sqrt{s})= & \sum_{V \in[16]} \frac{C_{V}^{(I, S, C)}}{8 m_{V}^{2}} \\
& \times\left(2 \sqrt{s}-M-\bar{M}+(\bar{M}-M) \frac{\bar{m}^{2}-m^{2}}{m_{V}^{2}}\right)
\end{aligned}
$$

is dropped. This was shown in [52] to be more consistent with the zero-range $(t \rightarrow 0)$ limit of the TVME employed in Ref. [50]. In the second place, a common heavy vectormeson mass $m_{H}$ and a common light vector-meson mass $m_{V}$ is used in the kernel, with the relation $m_{H} \sim 2 m_{V}$. In this way, the resulting interaction is of the same form as that shown in Eq. (13), namely,

$$
\begin{aligned}
V_{a b}^{I J S C}(\sqrt{s})= & \chi_{c} D_{a b}^{I J S C} \frac{2 \sqrt{s}-M_{a}-M_{b}}{4 f^{2}} \\
& \times \sqrt{\frac{E_{a}+M_{a}}{2 M_{a}}} \sqrt{\frac{E_{b}+M_{b}}{2 M_{b}}},
\end{aligned}
$$

but with an additional factor $\chi_{c}$, which is $\chi_{c}=1 / 4$ for charm-exchange transitions and $\chi_{c}=1$ otherwise. This factor accounts approximately for the ratio $\left(m_{V} / m_{H}\right)^{2}$ between the squared masses of uncharmed and charmed vector mesons, such as the $\rho$ and $D^{*}$, respectively. The additional scalar-isoscalar attractive interaction characterized by a $\Sigma_{D N}$ term, implemented in Ref. [52], is not considered here, since it would not affect the qualitative aspects of our results.

The main difference between the SU(4) models [49-52] and our SU(8) model, which is a must to be consistent with heavy-quark symmetry, is that the latter includes the vector 
meson-baryon channels. Another essential difference lies in the transition amplitudes between states containing heavy mesons because, according to Eq. (13), they scale with the inverse of a heavy-meson decay constant for each heavy meson involved, whereas in the SU(4) models the decay constant is kept fixed for all transitions. Therefore, as discussed at the end of Sec. II B, amplitudes such as $N D \rightarrow N D$ or $\Lambda D_{s} \rightarrow N D$ are typically a factor 3 smaller than those of the SU(4) models.

The results obtained within the SU(4) approach, Eq. (21), are collected in Table XIV, only for those IJ combinations that generate resonances. That excludes the $I=2, J=1 / 2$ sector, which has a repulsive interaction in the only allowed channel, $\Sigma_{c} \pi$; as well as the two-channel $\left(\Sigma_{c}^{*} \pi, \Delta D\right) I=2, J=3 / 2$ sector, where the mixing coefficient is not strong enough to overcome the repulsive $\Sigma_{c}^{*} \pi$ interaction. Note that we have slightly modified the subtraction point with a parameter $\alpha=0.959$, see Eq. (17), in order to place the $\Lambda(2595)$ resonance on the right energy. The generation of the resonance would have been facilitated if an attractive scalar-isoscalar $\Sigma_{D N}$ term was included, in which case the subtraction point would have to be modified by a value of $\alpha=0.979$, closer to one.

The main observation in the $I=0, J=1 / 2$ sector is that the model is able to generate a narrow resonance around $2600 \mathrm{MeV}$, which can be readily identified with the $\Lambda_{c}(2595)$. It couples strongly to $N D$ and its nature is therefore very different from the one obtained in the SU(8) model, which qualifies as being mainly a $N D^{*}$ quasibound state. The reason for the SU(4) model being able to generate the $\Lambda_{c}(2595)$ is that the lack of baryon-meson states in this sector (namely, those involving a vector meson and/ or a decuplet baryon) is somehow compensated by the enhanced strength in the baryon-meson channels involving a charmed meson. In other words, a resonance that has a strong $N D^{*}$ component in the $\mathrm{SU}(8)$ approach may reappear as a $N D$ state in the reduced SU(4) model space due to the enhanced interaction kernel. We also find relatively narrow resonances at 2799 and $3025 \mathrm{MeV}$ that couple, respectively, mostly to $\Xi_{c} K$ and $\Xi_{c}^{\prime} K$ states involving uncharmed meson channels and, therefore, are the analogous states as those obtained within the SU(8) model at 2821 and $3053 \mathrm{MeV}$, respectively (see Table IV), although the last state in the SU(4) model couples appreciably to $\Sigma_{c} \pi$ and $N D$, and hence is substantially wider. Logically the SU(4) model does not produce the resonances that couple strongly to states with light vector mesons in the SU(8) approach.

In the $I=1, J=1 / 2$ sector, we observe that the $\mathrm{SU}(4)$ model obtains a narrow resonance at $2661 \mathrm{MeV}$, which couples strongly to $N D$. Since the strength of this amplitude is reduced, the $\mathrm{SU}(8)$ model does not find such a type of resonance, but instead, a very narrow one at $2554 \mathrm{MeV}$ that couples strongly to $\Delta D^{*}$ and more moderately to $\Sigma^{*} D_{s}^{*}$ and $N D$, see Table VI. So again, while the two resonances do not differ that much in energy, their very nature is substantially different. The other relatively narrow resonance at $2957 \mathrm{MeV}$, coupling mainly to $\Sigma_{c} \eta$ and $\Xi_{c}^{\prime} K$, finds its analogue with the one at $2974 \mathrm{MeV}$ obtained in the SU(8) model, although this one has also vector-meson components of comparable size. The narrow states at $3158 \mathrm{MeV}$ and $3346 \mathrm{MeV}$, which couple mainly to vector-meson components and decay into $\Sigma_{c} \eta$ and $\Sigma D_{s}$ pairs, respectively, are not obtained in the SU(4) approach.

TABLE XIV. The same as Table IV, but for the SU(4) model, see more details in the text.

\begin{tabular}{|c|c|c|}
\hline$M_{R}$ & $\Gamma_{R}$ & Couplings to all SU(4) channels \\
\hline \multicolumn{3}{|r|}{$I=0, J=1 / 2$} \\
\hline $\begin{array}{l}2595.4 \\
2625.4 \\
2799.5 \\
3024.8\end{array}$ & $\begin{array}{r}2.01 \\
103.0 \\
0.0 \\
31.3\end{array}$ & $\begin{array}{c}\boldsymbol{g}_{\boldsymbol{\Sigma}_{c} \pi}=\mathbf{0 . 6 7}, g_{N D}=6.03, g_{\Lambda_{c} \eta}=0.12, g_{\Xi_{c} K}=0.07, g_{\Xi_{c}^{\prime} K}=0.17, g_{\Lambda D_{s}}=3.08, g_{\Lambda_{c} \eta^{\prime}}=0.29 \\
\boldsymbol{g}_{\mathbf{\Sigma}_{c} \pi}=\mathbf{2 . 3 0}, g_{N D}=1.55, g_{\Lambda_{c} \eta}=0.04, g_{\Xi_{c} K}=0.03, g_{\Xi_{c}^{\prime} K}=0.67, g_{\Lambda D_{s}}=1.05, g_{\Lambda_{c} \eta^{\prime}}=0.1 \\
\boldsymbol{g}_{\boldsymbol{\Sigma}_{c} \boldsymbol{\pi}}=\mathbf{0 . 3 5} \cdot \mathbf{1 0}-\mathbf{2}, g_{N D}=0.05, g_{\Lambda_{c} \eta}=1.47, g_{\Xi_{c} K}=2.57, g_{\Xi_{c}^{\prime} K}=0.02, g_{\Lambda D_{s}}=0.26, g_{\Lambda_{c} \eta^{\prime}}=0.02 \\
\boldsymbol{g}_{\boldsymbol{\Sigma}_{c} \boldsymbol{\pi}}=\mathbf{0 . 5 9}, \boldsymbol{g}_{N \boldsymbol{D}}=\mathbf{0 . 5 0}, \boldsymbol{g}_{\boldsymbol{\Lambda}_{c} \boldsymbol{\eta}}=\mathbf{0 . 1 6}, \boldsymbol{g}_{\Xi_{c} \boldsymbol{K}}=\mathbf{0 . 1 1}, g_{\Xi_{c}^{\prime} K}=2.22, g_{\Lambda D_{s}}=1.48, g_{\Lambda_{c} \eta^{\prime}}=0.02\end{array}$ \\
\hline \multicolumn{3}{|r|}{$I=1, J=1 / 2$} \\
\hline $\begin{array}{l}2661.2 \\
2694.7 \\
2938.0 \\
2956.5\end{array}$ & $\begin{array}{r}36.5 \\
153.0 \\
291.0 \\
29.5\end{array}$ & 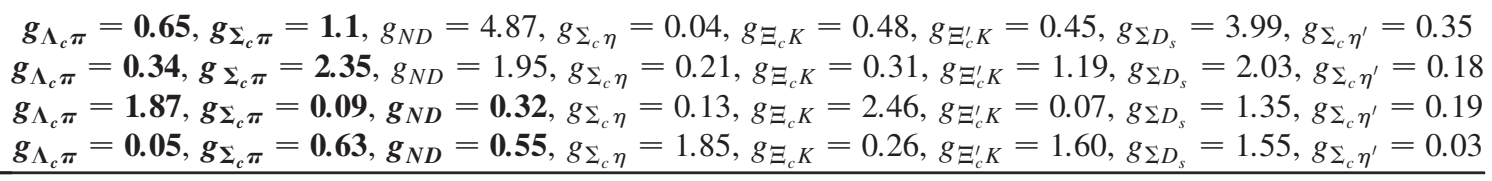 \\
\hline \multicolumn{3}{|r|}{$I=0, J=3 / 2$} \\
\hline $\begin{array}{l}2678.0 \\
3118.7\end{array}$ & $\begin{array}{l}82.7 \\
29.6\end{array}$ & $\begin{array}{l}\boldsymbol{g}_{\boldsymbol{\Sigma}_{c}^{*} \pi}=\mathbf{2 . 2 3}, g_{\Xi_{c}^{*} K}=0.61 \\
\boldsymbol{g}_{\boldsymbol{\Sigma}_{c}^{*} \pi}=\mathbf{0 . 6 7}, g_{\Xi_{c}^{*} K}=2.06\end{array}$ \\
\hline \multicolumn{3}{|r|}{$I=1, J=3 / 2$} \\
\hline $\begin{array}{l}2543.5 \\
2745.5 \\
3042.4\end{array}$ & $\begin{array}{r}0.0 \\
155.0 \\
31.2\end{array}$ & $\begin{array}{c}g_{\Sigma_{c}^{*} \pi}=0.22, g_{\Sigma_{c}^{*} \eta}=0.12, g_{\Delta D}=7.21, g_{\Xi_{c}^{*} K}=0.07, g_{\Sigma^{*} D_{s}}=3.27, g_{\Sigma_{c}^{*} \eta^{\prime}}=0.28 \\
\boldsymbol{g}_{\Sigma_{c}^{*} \pi}=\mathbf{2 . 2}, g_{\Sigma_{c}^{*} \eta}=0.22, g_{\Delta D}=0.45, g_{\Xi_{c}^{*} K}=1.09, g_{\Sigma^{*} D_{s}}=0.52, g_{\Sigma_{c}^{*} \eta^{\prime}}=0.06 \\
\boldsymbol{g}_{\Sigma_{c}^{*} \pi}=\mathbf{0 . 7 4}, g_{\Sigma_{c}^{*} \eta}=1.75, g_{\Delta D}=0.04, g_{\Xi_{c}^{*} K}=1.52, g_{\Sigma^{*} D_{s}}=0.07, g_{\Sigma_{c}^{*} \eta^{\prime}}=0.02\end{array}$ \\
\hline
\end{tabular}


In the $I=0, J=3 / 2$ sector, the SU(4) model produces resonances at $2678 \mathrm{MeV}$ and $3119 \mathrm{MeV}$ that couple mainly to $\Sigma_{c}^{*} \pi$ and $\Xi_{c}^{*} K$, respectively. The first one is equivalent to that found at $2659 \mathrm{MeV}$ in the $\mathrm{SU}(8)$ model, see Table. VIII. The second one could be identified basically with that appearing at $3094 \mathrm{MeV}$ in the $\mathrm{SU}(8)$ model that also has stronger vector-meson components. It has been argued in previous works [49,51] and in the present one, that the lowest-lying resonance could correspond to the observed three star $\Lambda_{c}(2625)$ of $J^{P}=3 / 2^{-}$, which is quoted to be the analogous in the charm sector of the $\Lambda(1520)$ [57]. The SU(8) model produces, apart from the state at $2940 \mathrm{MeV}$ that could be identified with the $\Lambda_{c}$ (2940), a few other narrow resonances beyond $3 \mathrm{GeV}$ that couple strongly to states containing vector mesons and, therefore, cannot be seen in the SU(4) model.

In the $I=1, J=3 / 2$ sector, there are three resonances that couple mainly to $\Delta D, \Sigma_{c}^{*} \pi$ and $\Sigma_{c}^{*} \eta$, respectively, and are the analogues of some of the lower energy states obtained in the SU(8) model, namely, those at $2550 \mathrm{MeV}$, $2687 \mathrm{MeV}$, and $3012 \mathrm{MeV}$. Note, however, that the resonance at $2550 \mathrm{MeV}$, which is the counterpart in the charm sector of the hyperonic $\Sigma(1670)$ resonance having mainly $\Delta \bar{K}$ components [29-31], becomes a $\Delta D^{*}$ resonance in the $\mathrm{SU}(8)$ model, although its coupling to $\Delta D, N D^{*}, \Sigma D_{s}^{*}$, and $\Sigma^{*} D_{s}^{*}$ is also significant. The three resonances obtained here with the simulated SU(4) model are the same as those found in Ref. [51], however with a notable exception for the width of the state around $3 \mathrm{GeV}$, which is quoted to be very narrow, while we find it to be around $30 \mathrm{MeV}$, due basically to a quite sizable coupling to $\Sigma_{c}^{*} \pi$ states. Note that, in this sector, the WT coefficient is -2 for the $\Sigma_{c}^{*} \pi$ transition and 0 for the $\Sigma_{c}^{*} \eta$, so the sizable coupling of this resonance to this latter channel is necessarily due to the nonperturbative coupled channels processes involving nondiagonal transitions.

We end this section by noting that the present simulation of the SU(4) model of Ref. [49] reproduces all the resonances given in Refs. [49,51] at approximately the same positions. Only the widths of some resonances appear to be wider here. This is because we are using an interaction kernel that ignores the tensor term of the $t$-channel vectormeson propagator, more consistent with the limit $t \rightarrow 0$, which is implicit in the contact WT interaction [52].

\section{CONCLUSIONS}

In the present work, we have studied charmed baryon resonances within a coupled channels unitary approach that implements, for the first time, the characteristic features of heavy-quark symmetry, as for instance the fact that $D$ and $D^{*}$ mesons have to be treated on an equal footing. This is accomplished by extending the $t$-channel vectorexchange SU(4) models (TVME) used in Refs. [49-52] to $\mathrm{SU}(8)$ spin-flavor symmetry, then by implementing a somewhat different way of breaking the flavor symmetry. More concretely, our tree-level $s$-wave WT amplitudes are obtained not only by adopting the physical hadron masses, but also by introducing the physical weak decay constants of the mesons involved in the transitions. This procedure reduces considerably the strength of diagonal amplitudes involving a charmed meson, as comparing to $\mathrm{SU}(4)$ models.

The present $\mathrm{SU}(8)$ model generates dynamically the resonances with negative parity in all the isospin-spin sectors that one can form from a $s$-wave interaction between the mesons of the $0^{-}, 1^{-}$multiplets with the $1 / 2^{+}$, $3 / 2^{+}$baryons. In this work we have focused only on the strangeness and charm quantum numbers appropriate for the $N D$ interaction, namely, $S=0$ and $C=1$. In the $I=$ $0, J=1 / 2$ sector, the lowest narrow state obtained can be readily identified with the $\Lambda_{c}(2595)$, which in our approach may be interpreted as being primarily a $N D^{*}$ bound state. We may also identify our state at $2660 \mathrm{MeV}$ in the $I=0, J=3 / 2$ sector, which couples strongly to the $\Sigma_{c}^{*} \pi$ channel, to be the three star $\Lambda_{c}$ (2625). The latter is usually referred to as being the charm counterpart of the $\Lambda(1520)$, which shows a strong coupling to $\Sigma^{*} \pi$ in dynamical models. Similarly, in the $I=1, J=3 / 2$ sector we find a resonance at $2550 \mathrm{MeV}$ that couples strongly to $\Delta D$ and $\Delta D^{*}$ states. This appears to be the counterpart of the $\Sigma$ (1670) hyperon, which couples to $\Delta \bar{K}$ in dynamical models. We also find in this sector a state at $2901 \mathrm{MeV}$ that could be a candidate for the $\Sigma_{c}(2800)$.

Our results have been compared with those obtained in the SU(4) models [49-52]. It appears that our SU(8) approach reproduces all the resonances generated in the SU(4) models, which couple strongly to the channels consisting of a pseudoscalar octet meson and a charmed baryon. They may be identified as chirally excited charmed baryons. On the other hand, due to the different pattern of flavor symmetry breaking, resonances in the SU(4) model that couple strongly to baryon-meson states containing a charmed meson and an uncharmed baryon (such as $N D$ and $\left.Y D_{s}\right)$ are, in principle, not necessarily reproduced within our SU(8) approach, which has adopted the physical decay constants, resulting in a reduced interaction in the corresponding transition amplitudes. However, the additional states implemented by the enlarged model space in SU(8) due to the requirement of heavy-quark symmetry, such as $N D^{*}$ and $\Delta D^{*}$, compensate largely for the reduced interaction, producing resonances in the same region or even more bound than those obtained in the SU(4) model, but with a quite different composition. A prime example is the $\Lambda_{c}(2595)$ in the $I=0, J=1 / 2$ sector. Within the $\mathrm{SU}(4)$ models this resonance is dynamically generated mainly from $N D$ states and it is then interpreted as a $N D$ quasibound state, with a mixture of $\Lambda D_{s}$ components, which lies very close to the threshold of its only decaying channel, $\Sigma_{c} \pi$. Instead, the SU(8) model interprets this resonance as being mainly a $N D^{*}$ quasibound state, although with sizable $N D, \Lambda D_{s}$ and $\Lambda D_{s}^{*}$ components. 
Our $\mathrm{SU}(8)$ approach predicts more states than those obtained within the SU(4) model. Not all of them find a direct identification with one of the observed resonances in the $C=1, S=0$ sector. We note, however, that many of our states couple weakly to the baryon-meson pairs from which the resonances are measured. It should also be pointed that some of our states would become wider or even disappear in a more realistic model containing also three-body channels and higher-multipolarity interactions. We finally note that the experimental spectra show a limited amount of counts and, in order to disentangle new resonant structures from data, more statistics is clearly needed.

\section{ACKNOWLEDGMENTS}

This work is partly supported by EU Contract No. FLAVIAnet MRTN-CT-2006-035482, by Contract Nos. FIS2008-01661/FIS and FIS2008-01143/FIS from MCNN (Spain), by the project CPAN CSD2007-00042 of the Program Consolider-Ingenio (Spain), and FEDER, by the Generalitat de Catalunya Contract No. 2005SGR00343, and Junta de Andalucía Grant Nos. FQM225, FQM481 and P06-FQM-01735. This research is part of the EU Integrated Infrastructure Initiative Hadron Physics Project under Contract No. RII3-CT-2004-506078. T. M. wishes to express his appreciation for the support of his visit to Barcelona through the research grants program of the Facultat de Física (University of Barcelona).

\section{APPENDIX A: HADRON SPIN-FLAVOR WAVE FUNCTIONS AND TENSOR METHOD}

This appendix is aimed for a detailed account of how to calculate the matrix element $D_{a b}^{I J S C}$ in the expression for the WT interaction, Eq. (13). It consists of defining the hadronic wavefunctions from the quark model and the tensor representation of the $\mathrm{SU}(8)$ operators and wave functions.

\section{Wavefunctions for the ground-state hadrons}

Pseudoscalar mesons

$$
\begin{array}{lll}
|\pi\rangle=-\frac{1}{\sqrt{2}}\left(Q_{u \uparrow}^{\dagger} Q_{\bar{d} \downarrow}^{\dagger}-Q_{u \downarrow}^{\dagger} Q_{\bar{d} \uparrow}^{\dagger}\right)|0\rangle, & |K\rangle=\frac{1}{\sqrt{2}}\left(Q_{u \uparrow}^{\dagger} Q_{\bar{s} \downarrow}^{\dagger}-Q_{u \downarrow}^{\dagger} Q_{\bar{s} \uparrow}^{\dagger}\right)|0\rangle, & |\bar{K}\rangle=-\frac{1}{\sqrt{2}}\left(Q_{s \uparrow}^{\dagger} Q_{\bar{d} \downarrow}^{\dagger}-Q_{s \downarrow}^{\dagger} Q_{\bar{d} \uparrow}^{\dagger}\right)|0\rangle, \\
|D\rangle=-\frac{1}{\sqrt{2}}\left(Q_{c \uparrow}^{\dagger} Q_{\bar{d} \downarrow}^{\dagger}-Q_{c \downarrow}^{\dagger} Q_{\bar{d} \uparrow}^{\dagger}\right)|0\rangle, & \left|D_{s}\right\rangle=\frac{1}{\sqrt{2}}\left(Q_{c \uparrow}^{\dagger} Q_{\bar{s} \downarrow}^{\dagger}-Q_{c \downarrow}^{\dagger} Q_{\bar{s} \uparrow}^{\dagger}\right)|0\rangle, & |\bar{D}\rangle=-\frac{1}{\sqrt{2}}\left(Q_{u \uparrow}^{\dagger} Q_{\bar{c} \downarrow}^{\dagger}-Q_{u \downarrow}^{\dagger} Q_{\bar{c} \uparrow}^{\dagger}\right)|0\rangle, \\
\left|\bar{D}_{s}\right\rangle=-\frac{1}{\sqrt{2}}\left(Q_{s \uparrow}^{\dagger} Q_{\bar{c} \downarrow}^{\dagger}-Q_{s \downarrow}^{\dagger} Q_{\bar{c} \uparrow}^{\dagger}\right)|0\rangle, \quad|\eta\rangle=\frac{1}{\sqrt{12}}\left(Q_{u \uparrow}^{\dagger} Q_{\bar{u} \downarrow}^{\dagger}-Q_{u \downarrow}^{\dagger} Q_{\bar{u} \uparrow}^{\dagger}+Q_{d \uparrow}^{\dagger} Q_{\bar{d} \downarrow}^{\dagger}-Q_{d \downarrow}^{\dagger} Q_{\bar{d} \uparrow}^{\dagger}-2 Q_{s \uparrow}^{\dagger} Q_{\bar{s} \downarrow}^{\dagger}+2 Q_{s \downarrow}^{\dagger} Q_{\bar{s} \uparrow}^{\dagger}\right)|0\rangle, \\
\left|\eta^{\prime}\right\rangle=\frac{1}{\sqrt{6}}\left(Q_{u \uparrow}^{\dagger} Q_{\bar{u} \downarrow}^{\dagger}-Q_{u \downarrow}^{\dagger} Q_{\bar{u} \uparrow}^{\dagger}+Q_{d \uparrow}^{\dagger} Q_{\bar{d} \downarrow}^{\dagger}-Q_{d \downarrow}^{\dagger} Q_{\bar{d} \uparrow}^{\dagger}+Q_{s \uparrow}^{\dagger} Q_{\bar{s} \downarrow}^{\dagger}-Q_{s \downarrow}^{\dagger} Q_{\bar{s} \uparrow}^{\dagger}\right)|0\rangle, & \left|\eta_{c}\right\rangle=\frac{1}{\sqrt{2}}\left(Q_{c \uparrow}^{\dagger} Q_{\bar{c} \downarrow}^{\dagger}-Q_{c \downarrow}^{\dagger} Q_{\bar{c} \uparrow}^{\dagger}\right)|0\rangle .
\end{array}
$$

Vector mesons

$$
\begin{array}{rlrl}
|\rho\rangle=-Q_{u \uparrow}^{\dagger} Q_{\bar{d} \uparrow}^{\dagger}|0\rangle, & \left|K^{*}\right\rangle=Q_{u \uparrow}^{\dagger} Q_{\bar{s} \mid}^{\dagger}|0\rangle, & \left|\bar{K}^{*}\right\rangle=-Q_{s \uparrow}^{\dagger} Q_{\bar{d} \uparrow}^{\dagger}|0\rangle, & \left|D^{*}\right\rangle=-Q_{c \uparrow}^{\dagger} Q_{\bar{d} \uparrow}^{\dagger}|0\rangle, \\
\left|D_{s}^{*}\right\rangle=Q_{c \uparrow}^{\dagger} Q_{\bar{s} \mid}^{\dagger}|0\rangle, & \left|\bar{D}^{*}\right\rangle=-Q_{u \uparrow}^{\dagger} Q_{\bar{c} \uparrow}^{\dagger}|0\rangle, & \left|\bar{D}_{s}^{*}\right\rangle=-Q_{s \uparrow}^{\dagger} Q_{\bar{c} \uparrow}^{\dagger}|0\rangle, & |\omega\rangle=\frac{1}{\sqrt{2}}\left(Q_{u \uparrow}^{\dagger} Q_{\bar{u} \uparrow}^{\dagger}+Q_{d \uparrow}^{\dagger} Q_{\bar{d} \uparrow}^{\dagger}\right)|0\rangle, \\
|\phi\rangle=-Q_{s \uparrow}^{\dagger} Q_{\bar{s} \uparrow}^{\dagger}|0\rangle, & |\psi\rangle=Q_{c \uparrow}^{\dagger} Q_{\bar{c} \uparrow}^{\dagger}|0\rangle . &
\end{array}
$$

Spin $1 / 2$ baryons

$$
\begin{aligned}
|\Lambda\rangle=\frac{1}{\sqrt{2}}\left(Q_{u \uparrow}^{\dagger} Q_{d \downarrow}^{\dagger} Q_{s \uparrow}^{\dagger}-Q_{u \downarrow}^{\dagger} Q_{d \uparrow}^{\dagger} Q_{s \uparrow}^{\dagger}\right)|0\rangle, & |N\rangle=\frac{1}{\sqrt{3}}\left(Q_{u \uparrow}^{\dagger} Q_{u \uparrow}^{\dagger} Q_{d \downarrow}^{\dagger}-Q_{u \uparrow}^{\dagger} Q_{u \downarrow}^{\dagger} Q_{d \uparrow}^{\dagger}\right)|0\rangle, \\
|\Sigma\rangle=\frac{1}{\sqrt{3}}\left(Q_{u \uparrow}^{\dagger} Q_{u \uparrow}^{\dagger} Q_{s \downarrow}^{\dagger}-Q_{u \uparrow}^{\dagger} Q_{u \downarrow}^{\dagger} Q_{s \uparrow}^{\dagger}\right)|0\rangle, & |\Xi\rangle=\frac{1}{\sqrt{3}}\left(Q_{u \uparrow}^{\dagger} Q_{s \downarrow}^{\dagger} Q_{s \uparrow}^{\dagger}-Q_{u \downarrow}^{\dagger} Q_{s \uparrow}^{\dagger} Q_{s \uparrow}^{\dagger}\right)|0\rangle, \\
\left|\Sigma_{c}\right\rangle=\frac{1}{\sqrt{3}}\left(Q_{u \uparrow}^{\dagger} Q_{u \uparrow}^{\dagger} Q_{c \downarrow}^{\dagger}-Q_{u \uparrow}^{\dagger} Q_{u \downarrow}^{\dagger} Q_{c \uparrow}^{\dagger}\right)|0\rangle, & \left|\Xi_{c}^{\prime}\right\rangle=\frac{1}{\sqrt{6}}\left(2 Q_{u \uparrow}^{\dagger} Q_{s \uparrow}^{\dagger} Q_{c \downarrow}^{\dagger}-Q_{u \uparrow}^{\dagger} Q_{s \downarrow}^{\dagger} Q_{c \uparrow}^{\dagger}-Q_{u \downarrow}^{\dagger} Q_{s \uparrow}^{\dagger} Q_{c \uparrow}^{\dagger}\right)|0\rangle, \\
\left|\Omega_{c}\right\rangle=\frac{1}{\sqrt{3}}\left(Q_{s \uparrow}^{\dagger} Q_{s \uparrow}^{\dagger} Q_{c \downarrow}^{\dagger}-Q_{s \uparrow}^{\dagger} Q_{s \downarrow}^{\dagger} Q_{c \uparrow}^{\dagger}\right)|0\rangle, & \left|\Xi_{c}\right\rangle=\frac{1}{\sqrt{2}}\left(Q_{u \uparrow}^{\dagger} Q_{s \downarrow}^{\dagger} Q_{c \uparrow}^{\dagger}-Q_{u \downarrow}^{\dagger} Q_{s \uparrow}^{\dagger} Q_{c \uparrow}^{\dagger}\right)|0\rangle, \\
\left|\Lambda_{c}\right\rangle=\frac{1}{\sqrt{2}}\left(Q_{u \uparrow}^{\dagger} Q_{d \downarrow}^{\dagger} Q_{c \uparrow}^{\dagger}-Q_{u \downarrow}^{\dagger} Q_{d \uparrow}^{\dagger} Q_{c \uparrow}^{\dagger}\right)|0\rangle, & \left|\Xi_{c c}\right\rangle=\frac{1}{\sqrt{3}}\left(Q_{u \uparrow}^{\dagger} Q_{c \downarrow}^{\dagger} Q_{c \uparrow}^{\dagger}-Q_{u \downarrow}^{\dagger} Q_{c \uparrow}^{\dagger} Q_{c \uparrow}^{\dagger}\right)|0\rangle, \\
\left|\Omega_{c c}\right\rangle=\frac{1}{\sqrt{3}}\left(Q_{s \uparrow}^{\dagger} Q_{c \downarrow}^{\dagger} Q_{c \uparrow}^{\dagger}-Q_{s \downarrow}^{\dagger} Q_{c \uparrow}^{\dagger} Q_{c \uparrow}^{\dagger}\right)|0\rangle . &
\end{aligned}
$$


Spin 3/2 baryons

$$
\begin{aligned}
|\Delta\rangle & =\frac{1}{\sqrt{6}} Q_{u \uparrow}^{\dagger} Q_{u \uparrow}^{\dagger} Q_{u \uparrow}^{\dagger}|0\rangle, & & \left|\Sigma^{*}\right\rangle=\frac{1}{\sqrt{2}} Q_{u \uparrow}^{\dagger} Q_{u \uparrow}^{\dagger} Q_{s \uparrow}^{\dagger}|0\rangle, \\
\left|\Sigma_{c}^{*}\right\rangle & =\frac{1}{\sqrt{2}} Q_{u \uparrow}^{\dagger} Q_{u \uparrow}^{\dagger} Q_{c \uparrow}^{\dagger}|0\rangle, & \left|\Xi_{c}^{*}\right\rangle & =Q_{u \uparrow}^{\dagger} Q_{s \uparrow}^{\dagger} Q_{c \uparrow}^{\dagger}|0\rangle, \\
\left|\Omega_{c c}^{*}\right\rangle & =\frac{1}{\sqrt{2}} Q_{s \uparrow}^{\dagger} Q_{c \uparrow}^{\dagger} Q_{c \uparrow}^{\dagger}|0\rangle, & \left|\Omega_{c c c}\right\rangle & =\frac{1}{\sqrt{6}} Q_{c \uparrow}^{\dagger} Q_{c \uparrow}^{\dagger} Q_{c \uparrow}^{\dagger}|0\rangle .
\end{aligned}
$$

$$
\begin{array}{r}
\left|\Xi^{*}\right\rangle=\frac{1}{\sqrt{2}} Q_{u \uparrow}^{\dagger} Q_{s \uparrow}^{\dagger} Q_{s \uparrow}^{\dagger}|0\rangle, \quad|\Omega\rangle=\frac{1}{\sqrt{6}} Q_{s \uparrow}^{\dagger} Q_{s \uparrow}^{\dagger} Q_{s \uparrow}^{\dagger}|0\rangle, \\
\left|\Omega_{c}^{*}\right\rangle=\frac{1}{\sqrt{2}} Q_{s \uparrow}^{\dagger} Q_{s \uparrow}^{\dagger} Q_{c \uparrow}^{\dagger}|0\rangle, \quad\left|\Xi_{c c}^{*}\right\rangle=\frac{1}{\sqrt{2}} Q_{u \uparrow}^{\dagger} Q_{c \uparrow}^{\dagger} Q_{c \uparrow}^{\dagger}|0\rangle,
\end{array}
$$

(Notes):

(i) Each state represents a member of a spin and isospin multiplet. The wavefunctions made explicit are those with the highest value of $I_{3}$ and $J_{3}$ in the multiplet.

(ii) $Q_{u \uparrow}^{\dagger}$ creates a $u$ quark with spin up, $Q_{\bar{d} \downarrow}^{\dagger}$ creates a quark $\bar{d}$ with spin down, and so on. As usual, color takes care of the fermionic statistics, so the quark operators are bosonic. The basis $\{|\uparrow\rangle,|\downarrow\rangle\}$ is of the standard SU(2) by which step operators $J_{ \pm}$have non-negative matrix elements. Also standard are $\{|u\rangle,|d\rangle\}, \quad\{|d\rangle,|s\rangle\}, \quad\{|s\rangle,|c\rangle\}, \quad$ and $\quad\{-|\bar{d}\rangle,|\bar{u}\rangle\}$, $\{-|\bar{s}\rangle,|\bar{d}\rangle\},\{-|\bar{c}\rangle,|\bar{s}\rangle\} .^{7}$ This means that the flavor$\mathrm{SU}(4)$ step operators act as

$$
\begin{array}{ll}
E_{12}|u\rangle=|d\rangle, & E_{23}|d\rangle=|s\rangle, \\
E_{34}|s\rangle=|c\rangle, & E_{12}|\bar{d}\rangle=-|\bar{u}\rangle, \\
E_{23}|\bar{s}\rangle=-|\bar{d}\rangle, & E_{34}|\bar{c}\rangle=-|\bar{s}\rangle .
\end{array}
$$

(iii) No special choice of relative phases has been made between different SU(4) multiplets. The relative phases of the hadronic states in an SU(4) multiplet are taken such that $E_{12}, E_{23}$, and $E_{34}$ have nonnegative matrix elements. Exceptions are the neutral mesons $\eta, \eta^{\prime}, \eta_{c}, \psi, \omega$, and $\phi$.

(iv) The pseudoscalar meson $\eta$ is (up to a sign) the isoscalar meson of the $\mathrm{SU}(3)$ octet and $\eta^{\prime}$ the $\mathrm{SU}(3)$ singlet. $\eta_{c}$ is purely $c \bar{c}$. In the spin 1 sector, $\psi$ is purely $c \bar{c}$, whereas $\phi$ and $\omega$ have been rotated in the $\mathrm{SU}(3)$ sector so that $\phi$ is purely $s \bar{s}$ (ideal mixing).

\section{Tensor representation for matrix elements}

Having identified the group structure of the extended WT interaction, the calculation of matrix elements can be done using standard group theoretical techniques. Methods based on computing $\mathrm{SU}(n)$ Clebsch-Gordan coefficients (e.g. [58]), extracting isoscalar factors (in SU(3) [76], and then $\mathrm{SU}(3)$-scalar factors in $\mathrm{SU}(4)$, etc.) are useful for explicit hand calculations for small groups but become more involved as the groups get larger. Instead, we follow a different route here. A tensor representation spanned by quark operators is used. This method only requires to know

${ }^{7}$ This is the convention used in [74,75] for arbitrary $\mathrm{SU}(n)$. Note that the convention in [76] for SU(3) is, instead, $\{|u\rangle,|d\rangle\}$, $\{|u\rangle,|s\rangle\},\{-|\bar{d}\rangle,|\bar{u}\rangle\},\{-|\bar{s}\rangle,|\bar{u}\rangle\}$.
Kronecker deltas and Wick contractions. Although it may not be practical for hand calculations, it is conceptually simple so that it is easy enough to automatize the calculation for larger groups.

Following [56] the WT Hamiltonian with spin-flavor symmetry takes the form

$$
-\mathcal{L}_{\mathrm{WT}}=\mathcal{H}_{\mathrm{WT}}=-\frac{\mathrm{i}}{4 f^{2}}:\left[\Phi, \partial_{0} \Phi\right]_{j}^{i} \mathcal{B}_{i \ell m}^{\dagger} \mathcal{B}^{j \ell m} .,
$$

where $\mathcal{B}^{i j k}$ is the baryon field, $\Phi^{i}{ }_{j}$ the meson field, and the $\mathrm{SU}(8)$ way of labeling the spin-flavor indices goes for $i, j$, etc. from 1 to 8 for four flavors. $\mathcal{B}^{i j k}$ is completely symmetric under permutation of indices. There is no universally accepted convention for the normalization. Here, we choose that $\mathcal{B}^{i j k}$ is normalized in a standard manner for a fermionic field when the indices $i, j, k$ are all different. Upon extracting the kinematical factors $(\sqrt{s}-M) /\left(2 f^{2}\right)$, the spin-flavor dependence is all contained in the matrices $D_{a b}^{I J}$. These are the matrix elements of the operator (see [56])

$$
\mathcal{G}=:\left(M^{i}{ }_{k} M^{\dagger k}{ }_{j}-M^{\dagger i}{ }_{k} M^{k}{ }_{j}\right) B^{\dagger}{ }_{i \ell m} B^{j \ell m},
$$

where $B^{i j k}$ and $B_{i j k}^{\dagger}$ denote the baryon annihilation and creation operators, normalized as

$$
\left[B^{i j k}, B_{i^{\prime} j^{\prime} k^{\prime}}^{\dagger}\right]=\delta_{i^{\prime}}^{i} \delta_{j^{\prime}}^{j} \delta_{k^{\prime}}^{k}+\delta_{j^{\prime}}^{i} \delta_{i^{\prime}}^{j} \delta_{k^{\prime}}^{k}+\cdots
$$

(3! permutations).

Likewise, $M_{j}^{i}$ and $M^{\dagger j}{ }_{i}=\left(M^{i}{ }_{j}\right)^{\dagger}$ denote the meson annihilation and creation operators, normalized as

$$
\left[M_{j}^{i}, M^{\dagger j^{\prime}}\right]=\delta_{i^{\prime}}^{i} \delta_{j^{\prime}}^{j}
$$

These characterizations automatically guarantee that the normalization and spin-flavor transformation properties of the states $\langle 0| B^{i j k}$ and $\langle 0| M^{i}{ }_{j}$ coincide with those of $\langle 0| Q^{i} Q^{j} Q^{k}$ and $\langle 0| Q^{i} \bar{Q}_{j}$, respectively. Here, $Q^{i}$ and $\bar{Q}_{i}$ are the $\mathrm{SU}(8)$ labeled annihilation operators of quarks and antiquarks, respectively. They transform as

$$
Q^{i} \rightarrow U^{i}{ }_{j} Q^{j}, \quad \bar{Q}_{i} \rightarrow\left(U^{i}{ }_{j}\right)^{*} \bar{Q}_{j}=\bar{Q}_{j}\left(U^{-1}\right)_{i}^{j}, \quad U \in \mathrm{SU}(8)
$$

and have standard normalization 


$$
\left[Q^{i}, Q^{\dagger}{ }_{j}\right]=\delta_{j}^{i}, \quad\left[\bar{Q}_{i}, \bar{Q}^{\dagger j}\right]=\delta_{i}^{j} .
$$

The correspondence between the $\mathrm{SU}(8)$ and the explicit spin-SU(4) flavor representations are the following: since $\bar{Q}_{i}$ transforms as $Q_{i}^{\dagger}$, the conventions in Sec. A 1 imply the identifications (up to a global sign)

$$
\begin{aligned}
& Q^{i}=\left(Q_{u \uparrow}, Q_{d \uparrow}, Q_{s \uparrow}, Q_{c \uparrow}, Q_{u \downarrow}, Q_{d \downarrow}, Q_{s \downarrow}, Q_{c \downarrow}\right), \\
& \bar{Q}_{i}=\left(Q_{\bar{u} \downarrow}, Q_{\bar{d} \downarrow}, Q_{\bar{s} \downarrow}, Q_{\bar{c} \downarrow},-Q_{\bar{u} \uparrow},-Q_{\bar{d} \uparrow},-Q_{\bar{s} \uparrow},-Q_{\bar{c} \uparrow}\right) .
\end{aligned}
$$

That is, $Q^{1}=Q_{u \uparrow}, \bar{Q}_{6}=-Q_{\bar{d} \uparrow}, \bar{Q}^{\dagger 6}=-Q_{\bar{d} \uparrow}^{\dagger}$, etc. The correct book keeping between an explicit flavor-spin index and the $\mathrm{SU}(8)$ one (running from 1 to 8 ) needs to be maintained to apply the Kronecker deltas in (A8) and (A9). Using these identifications together with

$$
Q_{i}^{\dagger} Q_{j}^{\dagger} Q_{k}^{\dagger} \rightarrow B_{i j k}^{\dagger}, \quad \bar{Q}^{\dagger i} Q_{j}^{\dagger} \rightarrow M^{\dagger i}{ }_{j},
$$

the mesonic and baryonic wavefunctions can be rewritten in terms of the operators $M_{j}^{\dagger i}$ and $B_{i j k}^{\dagger}$. For instance,

$$
\begin{aligned}
& |\pi\rangle=-\frac{1}{\sqrt{2}}\left(M_{1}^{\dagger 2}+M_{5}^{\dagger 6}\right)|0\rangle, \\
& |N\rangle=\frac{1}{\sqrt{3}}\left(B_{116}^{\dagger}-B_{152}^{\dagger}\right)|0\rangle
\end{aligned}
$$

(once again for the highest weights in the spin-isospin multiplet). It is straightforward now to obtain the matrix elements $\left\langle M B|G| M^{\prime} B^{\prime}\right\rangle$ using the commutation relations, or equivalently Wick's theorem. A nontrivial confirmation of the calculation is to see that the matrices $D_{a b}^{I J S C}$ so obtained have the correct eigenvalues $6,0,-2,-16$, -22 (the 0 being the consequence of the inclusion of the $\mathrm{SU}(8)$ singlet among the mesons). Another important point to check is that all the matrix elements computed are (up to a sign) square roots of rational numbers. While this is not surprising for matrix elements of $\mathrm{SU}(n)$ with standard bases, it is found to be the case also for the neutral vector mesons with ideal mixing.

\section{APPENDIX B: COEFFICIENTS OF THE $s$-WAVE TREE-LEVEL AMPLITUDES}

This appendix gives the coefficients $D_{a b}^{I J S C}$ of the $s$-wave

\begin{tabular}{|c|c|c|c|c|c|c|c|c|c|c|c|c|c|c|c|c|}
\hline & $\Sigma_{c} \pi$ & $N D$ & $\Lambda_{c} \eta$ & $N D^{*}$ & $\Xi_{c} K$ & $\Lambda_{c} \omega$ & $\Xi_{c}^{\prime} K$ & $\Lambda D_{s}$ & $\Lambda D_{s}^{*}$ & $\Sigma_{c} \rho$ & $\Lambda_{c} \eta^{\prime}$ & $\sum_{c}^{*} \rho$ & $\Lambda_{c} \phi$ & $\Xi_{c} K^{*}$ & $\Xi_{c}^{\prime} K^{*}$ & $\Xi_{c}^{*} K^{*}$ \\
\hline$\Sigma_{c} \pi$ & -4 & $\sqrt{\frac{3}{2}}$ & 0 & $-\sqrt{\frac{1}{2}}$ & 0 & 0 & $-\sqrt{3}$ & 0 & 0 & $-\sqrt{\frac{64}{3}}$ & 0 & $\sqrt{\frac{32}{3}}$ & 0 & $-\sqrt{3}$ & -2 & $\sqrt{2}$ \\
\hline$N D$ & $\sqrt{\frac{3}{2}}$ & -3 & $\sqrt{\frac{1}{2}}$ & $-\sqrt{27}$ & 0 & $\sqrt{\frac{9}{2}}$ & 0 & $-\sqrt{3}$ & -3 & $-\sqrt{\frac{1}{2}}$ & 1 & 2 & 0 & 0 & 0 & 0 \\
\hline$\Lambda_{c} \eta$ & 0 & $\sqrt{\frac{1}{2}}$ & 0 & $\sqrt{\frac{3}{2}}$ & $-\sqrt{3}$ & 0 & 0 & $-\sqrt{\frac{2}{3}}$ & $-\sqrt{2}$ & 0 & 0 & 0 & 0 & 0 & $-\sqrt{3}$ & $-\sqrt{6}$ \\
\hline$N D^{*}$ & $-\sqrt{\frac{1}{2}}$ & $-\sqrt{27}$ & $\sqrt{\frac{3}{2}}$ & -9 & 0 & $-\sqrt{\frac{3}{2}}$ & 0 & -3 & $-\sqrt{27}$ & $\sqrt{\frac{25}{6}}$ & $\sqrt{3}$ & $\sqrt{\frac{4}{3}}$ & 0 & 0 & 0 & 0 \\
\hline$\Xi_{c} K$ & 0 & 0 & $-\sqrt{3}$ & 0 & -2 & 0 & 0 & $\sqrt{\frac{1}{2}}$ & $\sqrt{\frac{3}{2}}$ & $-\sqrt{3}$ & 0 & $-\sqrt{6}$ & 0 & 0 & 0 & 0 \\
\hline$\Lambda_{c} \omega$ & 0 & $\sqrt{\frac{9}{2}}$ & 0 & $-\sqrt{\frac{3}{2}}$ & 0 & 0 & -1 & 0 & 0 & -4 & 0 & $\sqrt{8}$ & 0 & -1 & & $\sqrt{\frac{2}{3}}$ \\
\hline$\Xi_{c}^{\prime} K$ & $-\sqrt{3}$ & 0 & 0 & 0 & 0 & -1 & -2 & $\sqrt{\frac{3}{2}}$ & $-\sqrt{\frac{1}{2}}$ & -2 & 0 & $\sqrt{2}$ & $-\sqrt{2}$ & 0 & & $\sqrt{\frac{8}{3}}$ \\
\hline$\Lambda D_{s}$ & 0 & $-\sqrt{3}$ & $-\sqrt{\frac{2}{3}}$ & -3 & $\sqrt{\frac{1}{2}}$ & 0 & $\sqrt{\frac{3}{2}}$ & -1 & $-\sqrt{3}$ & 0 & $\sqrt{\frac{1}{3}}$ & 0 & $-\sqrt{3}$ & $\sqrt{\frac{3}{2}}$ & $-\sqrt{\frac{1}{2}}$ & 2 \\
\hline$\Lambda D_{s}^{*}$ & 0 & -3 & $-\sqrt{2}$ & $-\sqrt{27}$ & $\sqrt{\frac{3}{2}}$ & 0 & $-\sqrt{\frac{1}{2}}$ & $-\sqrt{3}$ & -3 & 0 & 1 & 0 & 1 & $-\sqrt{\frac{1}{2}}$ & $\sqrt{\frac{25}{6}}$ & $\sqrt{\frac{4}{3}}$ \\
\hline$\Sigma_{c} \rho$ & $-\sqrt{\frac{64}{3}}$ & $-\sqrt{\frac{1}{2}}$ & 0 & $\sqrt{\frac{25}{6}}$ & $-\sqrt{3}$ & -4 & -2 & 0 & 0 & $-\frac{20}{3}$ & 0 & $-\sqrt{\frac{8}{9}}$ & 0 & -2 & $-\sqrt{\frac{49}{3}}$ & $-\sqrt{\frac{2}{3}}$ \\
\hline$\Lambda_{c} \eta^{\prime}$ & 0 & 1 & 0 & $\sqrt{3}$ & 0 & 0 & 0 & $\sqrt{\frac{1}{3}}$ & 1 & 0 & 0 & 0 & 0 & 0 & 0 & 0 \\
\hline$\Sigma_{c}^{*} \rho$ & $\sqrt{\frac{32}{3}}$ & 2 & 0 & $\sqrt{\frac{4}{3}}$ & $-\sqrt{6}$ & $\sqrt{8}$ & $\sqrt{2}$ & 0 & 0 & $-\sqrt{\frac{8}{9}}$ & 0 & $-\frac{22}{3}$ & 0 & $\sqrt{2}$ & $-\sqrt{\frac{2}{3}}$ & $-\sqrt{\frac{64}{3}}$ \\
\hline$\Lambda_{c} \phi$ & 0 & 0 & 0 & 0 & 0 & 0 & $-\sqrt{2}$ & $-\sqrt{3}$ & 1 & 0 & 0 & 0 & 0 & $-\sqrt{2}$ & $\sqrt{\frac{8}{3}}$ & $-\sqrt{\frac{4}{3}}$ \\
\hline$\Xi_{c} K^{*}$ & $-\sqrt{3}$ & 0 & 0 & 0 & 0 & -1 & 0 & $\sqrt{\frac{3}{2}}$ & $-\sqrt{\frac{1}{2}}$ & -2 & 0 & $\sqrt{2}$ & $-\sqrt{2}$ & -2 & $-\sqrt{\frac{16}{3}}$ & $\sqrt{\frac{8}{3}}$ \\
\hline$\Xi_{c}^{\prime} K^{*}$ & -2 & 0 & $-\sqrt{3}$ & 0 & 0 & $-\sqrt{\frac{4}{3}}$ & $-\sqrt{\frac{16}{3}}$ & $-\sqrt{\frac{1}{2}}$ & $\sqrt{\frac{25}{6}}$ & $-\sqrt{\frac{49}{3}}$ & 0 & $-\sqrt{\frac{2}{3}}$ & $\sqrt{\frac{8}{3}}$ & $-\sqrt{\frac{16}{3}}$ & -2 & 0 \\
\hline$\Xi_{c}^{*} K^{*}$ & $\sqrt{2}$ & 0 & $-\sqrt{6}$ & 0 & 0 & $\sqrt{\frac{2}{3}}$ & $\sqrt{\frac{8}{3}}$ & 2 & $\sqrt{\frac{4}{3}}$ & & 0 & $-\sqrt{\frac{64}{3}}$ & $-\sqrt{\frac{4}{3}}$ & $\sqrt{\frac{8}{3}}$ & 0 & -2 \\
\hline
\end{tabular}
tree-level baryon-meson ${ }^{8}$ amplitudes of Eq. (10) for the various IJSC sectors, in the particular case of $S=0$ and $C=1$. They are shown in Tables XV, XVI, XVII, XVIII, XIX, XX, XXI, XXII, and XXIII.

TABLE XV. $I=0, J=1 / 2, S=0, C=1$. Baryon-meson states with more than one $c$ quark have not been included.

\footnotetext{
${ }^{8} \mathrm{We}$ say baryon-meson amplitudes to stress that the given $D^{I J S C}$ matrix elements corresponds to the state of the two colliding hadrons coupled in this order: baryon meson. Change of phases in the matrix elements can be derived of using other order.
} 
TABLE XVI. $I=1, J=1 / 2, S=0, C=1$. Baryon-meson states with more than one $c$ quark have not been included.

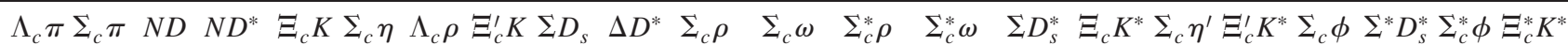

\begin{tabular}{lllllllllllllllllllllll}
\hline$\Lambda_{c} \pi$ & 0 & 0 & $\sqrt{\frac{3}{2}}$ & $\sqrt{\frac{9}{2}}$ & 1 & 0 & 0 & 0 & 0 & 0 & $\sqrt{8}$ & 0 & 4 & 0 & 0 & 0 & 0 & 1 & 0 & 0 & 0 & $\sqrt{2}$
\end{tabular}

$\begin{array}{lllllllllllllllllllll}\Sigma_{c} \pi & 0 & -2 & 1 & -\sqrt{\frac{1}{3}} & 0 & 0 & \sqrt{8}-\sqrt{2} & 0 & \sqrt{\frac{8}{3}}-\sqrt{\frac{16}{3}} & 0 & \sqrt{\frac{8}{3}} & 0 & 0 & -\sqrt{2} & 0 & -\sqrt{\frac{8}{3}} & 0 & 0 & 0 & \sqrt{\frac{4}{3}}\end{array}$

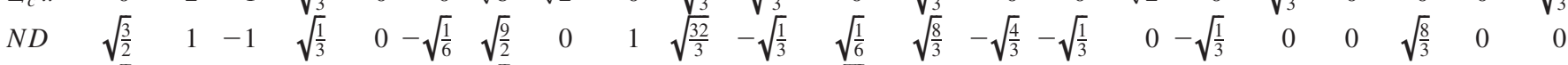

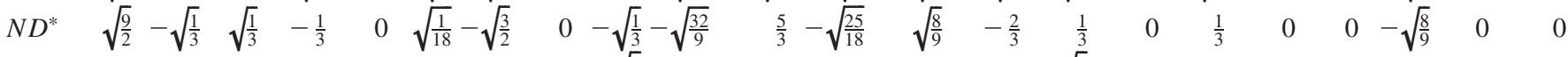

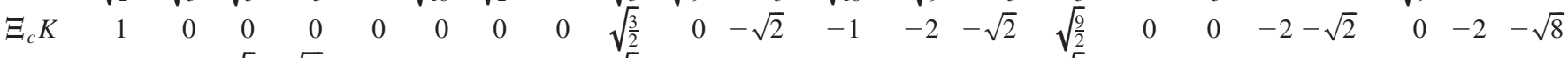

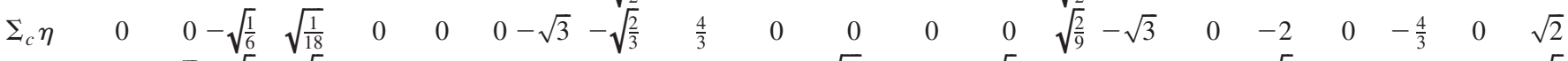

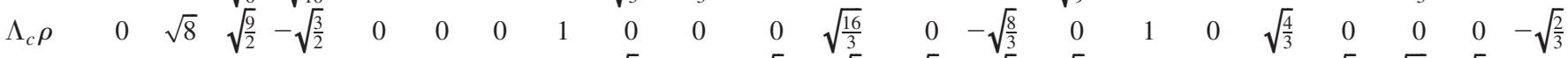

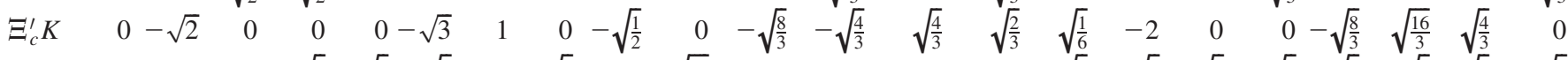

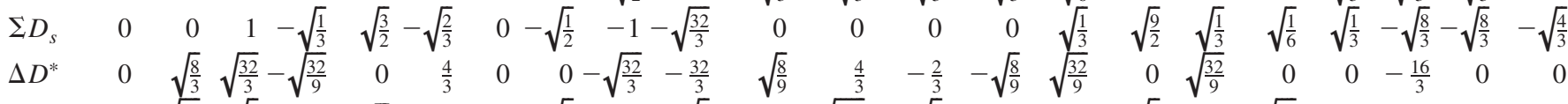

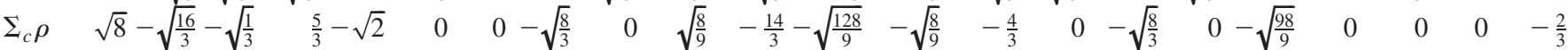

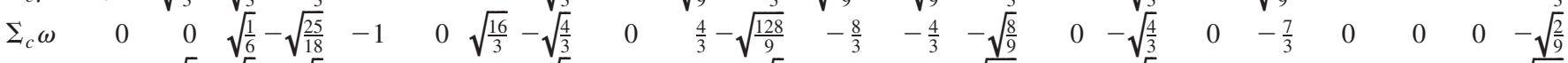

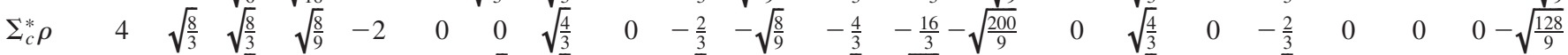

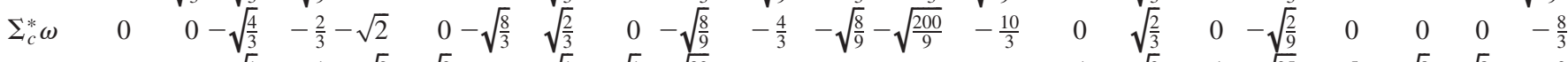

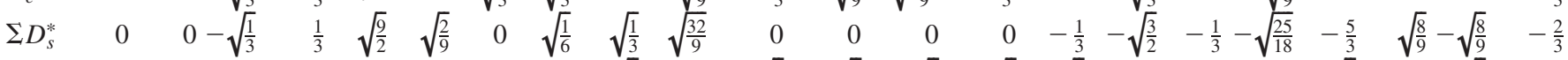

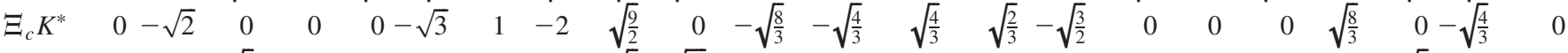

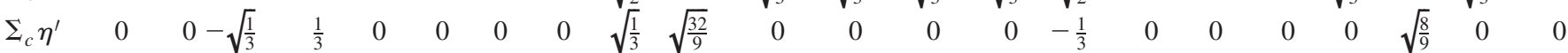

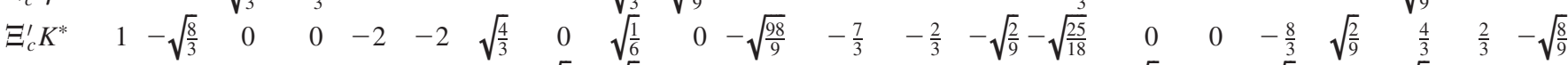

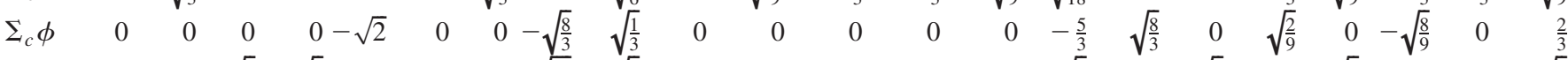

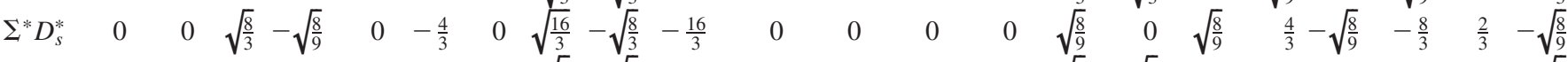

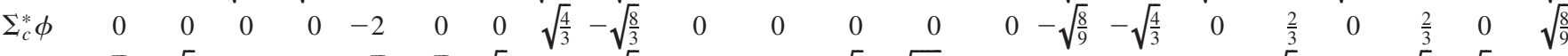

\begin{tabular}{llllllllllllllllllll}
$\Xi_{c}^{*} K^{*}$ & $\sqrt{2}$ & $\sqrt{\frac{4}{3}}$ & 0 & $0-\sqrt{8}$ & $\sqrt{2}-\sqrt{\frac{2}{3}}$ & 0 & $-\sqrt{\frac{4}{3}}$ & 0 & $-\frac{2}{3}$ & $-\sqrt{\frac{2}{9}}-\sqrt{\frac{128}{9}}$ & $-\frac{8}{3}$ & $-\frac{2}{3}$ & 0 & 0 & $-\sqrt{\frac{8}{9}}$ & $\frac{2}{3}$ & $-\sqrt{\frac{8}{9}}$ & $\sqrt{\frac{8}{9}}$ & $-\frac{10}{3}$ \\
\hline
\end{tabular}

TABLE XVII. $I=2, J=1 / 2, S=0, C=1$. Baryon-meson states with more than one $c$ quark have not been included.

\begin{tabular}{lrrrr}
\hline \hline & $\Sigma_{c} \pi$ & $\Delta D^{*}$ & $\Sigma_{c} \rho$ & $\Sigma_{c}^{*} \rho$ \\
\hline$\Sigma_{c} \pi$ & 2 & $\sqrt{8}$ & $\sqrt{\frac{16}{3}}$ & $-\sqrt{\frac{8}{3}}$ \\
$\Delta D^{*}$ & $\sqrt{8}$ & 0 & $\sqrt{\frac{8}{3}}$ & $-\sqrt{\frac{4}{3}}$ \\
$\Sigma_{c} \rho$ & $\sqrt{\frac{16}{3}}$ & $\sqrt{\frac{8}{3}}$ & $-\frac{2}{3}$ & $-\sqrt{\frac{8}{9}}$ \\
$\Sigma_{c}^{*} \rho$ & $-\sqrt{\frac{8}{3}}$ & $-\sqrt{\frac{4}{3}}$ & $-\sqrt{\frac{8}{9}}$ & $-\frac{4}{3}$ \\
\hline \hline
\end{tabular}


TABLE XVIII. $I=0, J=3 / 2, S=0, C=1$. Baryon-meson states with more than one $c$ quark have not been included.

\begin{tabular}{|c|c|c|c|c|c|c|c|c|c|c|c|}
\hline & $\Sigma_{c}^{*} \pi$ & $N D^{*}$ & $\Lambda_{c} \omega$ & $\Xi_{c}^{*} K$ & $\Lambda D_{s}^{*}$ & $\Sigma_{c} \rho$ & $\Sigma_{c}^{*} \rho$ & $\Lambda_{c} \phi$ & $\Xi_{c} K^{*}$ & $\Xi_{c}^{\prime} K^{*}$ & $\Xi_{c}^{*} K^{*}$ \\
\hline$\Sigma_{c}^{*} \pi$ & -4 & $-\sqrt{2}$ & 0 & $-\sqrt{3}$ & 0 & $-\sqrt{\frac{16}{3}}$ & $-\sqrt{\frac{80}{3}}$ & 0 & $\sqrt{3}$ & -1 & $-\sqrt{5}$ \\
\hline$N D^{*}$ & $-\sqrt{2}$ & 0 & $\sqrt{6}$ & 0 & 0 & $\sqrt{\frac{2}{3}}$ & $\sqrt{\frac{10}{3}}$ & 0 & 0 & 0 & 0 \\
\hline$\Lambda_{c} \omega$ & 0 & $\sqrt{6}$ & 0 & 1 & 0 & 2 & $\sqrt{20}$ & 0 & -1 & $\sqrt{\frac{1}{3}}$ & $\sqrt{\frac{5}{3}}$ \\
\hline$\Xi_{c}^{*} K$ & $-\sqrt{3}$ & 0 & 1 & -2 & $-\sqrt{2}$ & -1 & $-\sqrt{5}$ & $\sqrt{2}$ & 0 & $-\sqrt{\frac{4}{3}}$ & $-\sqrt{\frac{20}{3}}$ \\
\hline$\Lambda D_{s}^{*}$ & 0 & 0 & 0 & $-\sqrt{2}$ & 0 & 0 & 0 & -2 & $\sqrt{2}$ & $\sqrt{\frac{2}{3}}$ & $\sqrt{\frac{10}{3}}$ \\
\hline$\Sigma_{c} \rho$ & $-\sqrt{\frac{16}{3}}$ & $\sqrt{\frac{2}{3}}$ & 2 & -1 & 0 & $-\frac{8}{3}$ & $-\sqrt{\frac{20}{9}}$ & 0 & 1 & $-\sqrt{\frac{1}{3}}$ & $-\sqrt{\frac{5}{3}}$ \\
\hline$\Sigma_{c}^{*} \rho$ & $-\sqrt{\frac{80}{3}}$ & $\sqrt{\frac{10}{3}}$ & $\sqrt{20}$ & $-\sqrt{5}$ & 0 & $-\sqrt{\frac{20}{9}}$ & $-\sqrt{\frac{16}{3}}$ & 0 & $\sqrt{5}$ & $-\sqrt{\frac{5}{3}}$ & $-\sqrt{\frac{25}{3}}$ \\
\hline$\Lambda_{c} \phi$ & 0 & 0 & 0 & $\sqrt{2}$ & -2 & 0 & 0 & 0 & $-\sqrt{2}$ & $-\sqrt{\frac{2}{3}}$ & $-\sqrt{\frac{10}{3}}$ \\
\hline$\Xi_{c} K^{*}$ & $\sqrt{3}$ & 0 & -1 & 0 & $\sqrt{2}$ & 1 & $\sqrt{5}$ & $-\sqrt{2}$ & -2 & $\sqrt{\frac{4}{3}}$ & $\sqrt{\frac{20}{3}}$ \\
\hline$\Xi_{c}^{\prime} K^{*}$ & -1 & 0 & $\sqrt{\frac{1}{3}}$ & $-\sqrt{\frac{4}{3}}$ & $\sqrt{\frac{2}{3}}$ & $-\sqrt{\frac{1}{3}}$ & $-\sqrt{\frac{5}{3}}$ & $-\sqrt{\frac{2}{3}}$ & $\sqrt{\frac{4}{3}}$ & -2 & 0 \\
\hline$\Xi_{c}^{*} K^{*}$ & $-\sqrt{5}$ & 0 & $\sqrt{\frac{5}{3}}$ & $-\sqrt{\frac{20}{3}}$ & $\sqrt{\frac{10}{3}}$ & $-\sqrt{\frac{5}{3}}$ & $-\sqrt{\frac{25}{3}}$ & $-\sqrt{\frac{10}{3}}$ & $\sqrt{\frac{20}{3}}$ & 0 & -2 \\
\hline
\end{tabular}

TABLE XIX. $I=1, J=3 / 2, S=0, C=1$. Baryon-meson states with more than one $c$ quark have not been included.

\begin{tabular}{|c|c|c|c|c|c|c|c|c|c|c|c|c|c|c|}
\hline & $\sum_{c}^{*} \pi N D^{*}$ & $\Lambda_{c} \rho$ & $\Sigma_{c}^{*} \eta$ & $\Delta D$ & $\Xi_{c}^{*} K$ & $\Delta D^{*}$ & $\Sigma_{c} \rho$ & $\Sigma_{c} \omega \Sigma$ & & $\Sigma D_{s}^{*}$ & $\sum^{*} D_{s} \Xi_{c} K^{*}$ & $\Xi_{c}^{\prime} K^{*} \Sigma_{c} \phi$ & $\Sigma_{c}^{*} \eta^{\prime} \Sigma^{*} D_{s}^{*} \Sigma_{c}^{*} \phi$ & $\Xi$ \\
\hline$\Sigma_{c}^{*} \pi$ & $-2-\sqrt{\frac{4}{3}}$ & $\frac{\sqrt{4}}{3}-\sqrt{8}$ & 0 & & $1-\sqrt{2}$ & $\sqrt{\frac{5}{3}}$ & & $0-\sqrt{\frac{20}{3}}$ & $\frac{\overline{0}}{3}$ & 0 & $\sqrt{2}$ & $-\sqrt{\frac{2}{3}}$ & 0 & \\
\hline$N D^{*}$ & $-\sqrt{\frac{4}{3}} \quad-\frac{4}{3}$ & $\sqrt{6}$ & $\sqrt{\frac{2}{9}}$ & & & $-\sqrt{\frac{80}{9}}$ & & $\sqrt{\frac{2}{9}} \quad \sqrt{\frac{20}{9}}$ & $\sqrt{\frac{10}{9}}$ & $\frac{4}{3}$ & $-\sqrt{\frac{4}{3}}$ & 0 & $\frac{2}{3}-\sqrt{\frac{20}{9}}$ & ) \\
\hline$\Lambda_{c} \rho$ & $-\sqrt{8}$ & $\overline{6}$ & 0 & & -1 & 0 & 0 & $-\sqrt{\frac{4}{3}}$ & $\sqrt{\frac{20}{3}}$ & 0 & 0 & $-\sqrt{\frac{1}{3}}$ & 0 & $-\sqrt{\frac{5}{3}}$ \\
\hline$\Sigma_{c}^{*} \eta$ & 0 & 0 & 0 & & $\sqrt{\frac{2}{3}}-\sqrt{3}$ & $\sqrt{\frac{10}{9}}$ & & 0 & 0 & $\sqrt{\frac{8}{9}}$ & $-\sqrt{\frac{2}{3}}$ & -1 & $0-\sqrt{\frac{10}{9}}$ & $-\sqrt{5}$ \\
\hline$\Delta D$ & $1-\sqrt{\frac{16}{3}}$ & $\frac{6}{3}$ & $\sqrt{\frac{2}{3}}$ & & & $-\sqrt{\frac{80}{3}}$ & & $-\sqrt{\frac{8}{3}}$ & $\sqrt{\frac{10}{3}}$ & $\sqrt{\frac{10}{3}}$ & -2 & 0 & $\sqrt{\frac{4}{3}}-\sqrt{\frac{20}{3}}$ & 0 \\
\hline$\Xi_{c}^{*} K$ & $-\sqrt{2}$ & -1 & $-\sqrt{3}$ & & 0 & 0 & 0 & $\sqrt{\frac{10}{3}}$ & $-\sqrt{\frac{5}{3}}$ & $\sqrt{\frac{2}{3}}$ & $\sqrt{2}$ & $-\sqrt{\frac{2}{3}}$ & $\sqrt{\frac{10}{3}}$ & $\frac{5}{2}$ \\
\hline$\Delta D^{*}$ & $\sqrt{\frac{5}{3}}-\sqrt{\frac{80}{9}}$ & $\frac{80}{9}$ & $\sqrt{\frac{10}{9}}$ & $-\sqrt{\frac{8}{3}}$ & $\frac{80}{3}$ & $-\frac{20}{3}$ & $\sqrt{\frac{21}{9}}$ & $\sqrt{\frac{40}{9}}$ & $\sqrt{\frac{2}{9}}$ & $\sqrt{\frac{80}{9}}$ & $-\sqrt{\frac{20}{3}}$ & 0 & $-\frac{10}{3}$ & 0 \\
\hline$\Sigma_{c} \rho$ & $-\sqrt{\frac{4}{3}}$ & $\frac{2}{3} \quad 0$ & 0 & $-\sqrt{ }$ & & $\sqrt{\frac{20}{9}}$ & - & $\sqrt{\frac{32}{9}}-\sqrt{\frac{20}{9}}$ & $\frac{0}{9}-\sqrt{\frac{40}{9}}$ & 0 & $\sqrt{\frac{2}{3}}$ & $-\sqrt{\frac{2}{9}}$ & 0 & \\
\hline$\Sigma_{c} \omega$ & $-\sqrt{\frac{2}{9}}$ & $\frac{2}{\frac{2}{9}}-\sqrt{\frac{4}{3}}$ & 0 & $-\sqrt{ }$ & $-\sqrt{\frac{1}{3}}$ & $\sqrt{\frac{40}{9}}$ & $\sqrt{\frac{3}{9}}$ & $\frac{4}{3}-\sqrt{\frac{40}{9}}$ & $\frac{0}{9}-\sqrt{\frac{20}{9}}$ & 0 & $\sqrt{\frac{1}{3}}$ & $-\frac{1}{3}$ & 0 & ) \\
\hline$\sum_{c}^{*} \rho$ & $-\sqrt{\frac{20}{3}} \quad \sqrt{\frac{20}{9}}$ & $\begin{array}{ll}\frac{0}{9} & 0 \\
\end{array}$ & 0 & & $\sqrt{\frac{5}{3}}-\sqrt{\frac{10}{3}}$ & & $\frac{1}{3}-\sqrt{\frac{22}{9}}$ & $\frac{20}{9}-\sqrt{\frac{40}{9}}-\frac{10}{3}$ & $-\sqrt{\frac{32}{9}}$ & 0 & $\sqrt{\frac{10}{3}}$ & {$\left[-\sqrt{\frac{10}{9}}\right.$} & 0 & \\
\hline$\Sigma_{c}^{*} \omega$ & $0-\sqrt{\frac{10}{9}}$ & $\frac{0}{9}-\sqrt{\frac{20}{3}}$ & 0 & $\sqrt{\frac{1}{3}}$ & $\frac{10}{3}-\sqrt{\frac{5}{3}}$ & $\sqrt{\frac{2}{9}}$ & $\frac{2}{9}-\sqrt{\frac{4}{9}}$ & $\frac{40}{9}-\sqrt{\frac{20}{9}}-\sqrt{\frac{32}{9}}$ & $-\frac{4}{3}$ & 0 & $\sqrt{\frac{5}{3}}$ & $-\sqrt{\frac{5}{9}}$ & 0 & $-\frac{5}{3}$ \\
\hline$\Sigma D_{s}^{*}$ & $0 \quad \frac{4}{3}$ & $\frac{4}{3}$ & $\sqrt{\frac{8}{9}}$ & $\sqrt{\frac{1}{3}}$ & $\sqrt{\frac{2}{3}}$ & $\sqrt{\frac{80}{9}}$ & & 0 & 0 & $-\frac{4}{3}$ & $\sqrt{6}$ & $-\sqrt{\frac{2}{9}}$ & $\sqrt{\frac{20}{9}}-\sqrt{\frac{20}{9}}$ & $\sqrt{\frac{10}{9}}$ \\
\hline$\Sigma^{*} D_{s}$ & $0-\sqrt{\frac{4}{3}}$ & $\sqrt{\frac{4}{3}}$ & $-\sqrt{\frac{2}{3}}$ & - & $\sqrt{2}$ & $-\sqrt{\frac{20}{3}}$ & & 0 & 0 & $\sqrt{\frac{4}{3}}$ & -1 & $-\sqrt{\frac{8}{3}}$ & $-\sqrt{\frac{5}{3}}-\sqrt{\frac{5}{3}}$ & $\sqrt{\frac{10}{3}}$ \\
\hline$\Xi_{c} K^{*}$ & $\sqrt{2}$ & 0 & $\sqrt{3}$ & & 0 & 0 & $\sqrt{3}$ & $\sqrt{\frac{1}{3}}$ & $\sqrt{\frac{5}{3}}$ & $\sqrt{6}$ & 0 & $0-\sqrt{\frac{2}{3}}$ & $0-\sqrt{\frac{10}{3}}$ & 0 \\
\hline$\Xi_{c}^{\prime} K^{*}$ & $-\sqrt{\frac{2}{3}}$ & $0-\sqrt{\frac{1}{3}}$ & -1 & & 0 & 0 & $0-\sqrt{3}$ & $-\frac{1}{3}-\sqrt{\frac{10}{9}}$ & $\sqrt{\frac{5}{9}}$ & $-\sqrt{\frac{2}{9}}$ & $-\sqrt{\frac{8}{3}}$ & $-\sqrt{\frac{50}{9}}$ & $\sqrt{\frac{40}{9}}$ & $-\sqrt{\frac{20}{9}}$ \\
\hline$\Sigma_{c} \phi$ & 0 & 0 & 0 & & $0-\sqrt{\frac{2}{3}}$ & 0 & 0 & $\begin{array}{ll}0 & 0\end{array}$ & 0 & $-\frac{2}{3}$ & $\sqrt{\frac{4}{3}}$ & $-\sqrt{\frac{50}{9}}$ & $0-\sqrt{\frac{20}{9}}$ & $\sqrt{\frac{10}{9}}$ \\
\hline$\Sigma_{c}^{*} \eta^{\prime}$ & 0 & $\frac{2}{3}$ & 0 & & $\sqrt{\frac{4}{3}} \quad 0$ & $\sqrt{\frac{20}{9}}$ & & 0 & 0 & $-\frac{2}{3}$ & $\sqrt{\frac{1}{3}}$ & 0 & $\sqrt{\frac{5}{9}}$ & 0 \\
\hline$\Sigma^{*} D_{s}^{*}$ & $0-\sqrt{\frac{20}{9}}$ & & $-\sqrt{\frac{10}{9}}$ & & $\begin{array}{ll}\frac{20}{3} & \sqrt{\frac{10}{3}}\end{array}$ & $-\frac{10}{3}$ & & 0 & 0 & $0 \quad \sqrt{\frac{20}{9}}$ & $-\sqrt{\frac{5}{3}}$ & $\sqrt{\frac{40}{9}}-\sqrt{\frac{20}{9}}$ & $\begin{array}{ll}-\frac{5}{3} & -\frac{1}{3}\end{array}$ & $\sqrt{\frac{2}{9}}$ \\
\hline$\Sigma_{c}^{*} \phi$ & 0 & 0 & 0 & & $0-\sqrt{\frac{10}{3}}$ & 0 & 0 & 0 & & $0-\sqrt{\frac{20}{9}}$ & $-\sqrt{\frac{5}{3}}-\sqrt{\frac{10}{3}}$ & $\sqrt{\frac{10}{9}} \quad 0$ & $-\frac{1}{3}$ & $-\sqrt{\frac{2}{9}}$ \\
\hline$\Xi_{c}^{*} K^{*}$ & $-\sqrt{\frac{10}{3}}$ & $0-\sqrt{\frac{5}{3}}$ & $-\sqrt{5}$ & & $0 \quad 0$ & & $0-\sqrt{\frac{11}{9}}$ & $\frac{10}{9}-\sqrt{\frac{5}{9}}-\sqrt{\frac{50}{9}}$ & & $\frac{5}{3}-\sqrt{\frac{10}{9}}$ & $\sqrt{\frac{10}{3}}$ & $-\sqrt{\frac{20}{9}}$ & $\sqrt{\frac{2}{9}}-\sqrt{\frac{2}{9}}$ & $-\frac{4}{3}$ \\
\hline
\end{tabular}


$s$-WAVE CHARMED BARYON RESONANCES FROM A $\ldots$

TABLE XX. $I=2, J=3 / 2, S=0, C=1$. Baryon-meson states with more than one $c$ quark have not been included.

\begin{tabular}{lrrrrr}
\hline \hline & $\Sigma_{c}^{*} \pi$ & $\Delta D$ & $\Delta D^{*}$ & $\Sigma_{c} \rho$ & $\Sigma_{c}^{*} \rho$ \\
\hline$\sum_{c}^{*} \pi$ & 2 & $\sqrt{3}$ & $\sqrt{5}$ & $\sqrt{\frac{4}{3}}$ & $\sqrt{\frac{20}{3}}$ \\
$\Delta D$ & $\sqrt{3}$ & 0 & 0 & -2 & $\sqrt{5}$ \\
$\Delta D^{*}$ & $\sqrt{5}$ & 0 & 0 & $\sqrt{\frac{20}{3}}$ & $\sqrt{\frac{1}{3}}$ \\
$\sum_{c} \rho$ & $\sqrt{\frac{4}{3}}$ & -2 & $\sqrt{\frac{20}{3}}$ & $\sqrt{\frac{10}{3}}$ & $-\sqrt{\frac{20}{9}}$ \\
$\sum_{c}^{*} \rho$ & $\sqrt{\frac{20}{3}}$ & $\sqrt{5}$ & $\sqrt{\frac{1}{3}}$ & $-\sqrt{\frac{20}{9}}$ & $\frac{2}{3}$ \\
\hline \hline
\end{tabular}

TABLE XXI. $I=0, J=5 / 2, S=0, C=1$. Baryon-meson states with more than one $c$ quark have not been included.

\begin{tabular}{lcc}
\hline \hline & $\sum_{c}^{*} \rho$ & $\Xi_{c}^{*} K^{*}$ \\
\hline$\sum_{c}^{*} \rho$ & -2 & 0 \\
$\Xi_{c}^{*} K^{*}$ & 0 & -2 \\
\hline \hline
\end{tabular}

[1] J. A. Oller, E. Oset, and A. Ramos, Prog. Part. Nucl. Phys. 45, 157 (2000).

[2] N. Kaiser, P. B. Siegel, and W. Weise, Nucl. Phys. A594, 325 (1995).

[3] N. Kaiser, P. B. Siegel, and W. Weise, Phys. Lett. B 362, 23 (1995).

[4] N. Kaiser, T. Waas, and W. Weise, Nucl. Phys. A612, 297 (1997).

[5] E. Oset and A. Ramos, Nucl. Phys. A635, 99 (1998).

[6] B. Krippa, Phys. Rev. C 58, 1333 (1998); B. Krippa and J. T. Londergan, Phys. Rev. C 58, 1634 (1998).

[7] J. C. Nacher, A. Parreno, E. Oset, A. Ramos, A. Hosaka, and M. Oka, Nucl. Phys. A678, 187 (2000).

[8] U. G. Meissner and J.A. Oller, Nucl. Phys. A673, 311 (2000).

[9] J.A. Oller and U. G. Meissner, Phys. Lett. B 500, 263 (2001).

[10] J. Nieves and E. Ruiz Arriola, Phys. Rev. D 64, 116008 (2001).

[11] T. Inoue, E. Oset, and M. J. Vicente Vacas, Phys. Rev. C 65, 035204 (2002).

[12] M. F. M. Lutz and E. E. Kolomeitsev, Nucl. Phys. A700, 193 (2002).

[13] C. Garcia-Recio, J. Nieves, E. Ruiz Arriola, and M.J. Vicente Vacas, Phys. Rev. D 67, 076009 (2003).

[14] E. Oset, A. Ramos, and C. Bennhold, Phys. Lett. B 527, 99 (2002).

[15] A. Ramos, E. Oset, and C. Bennhold, Phys. Rev. Lett. 89, 252001 (2002).

[16] J. A. Oller, J. Prades, and M. Verbeni, Phys. Rev. Lett. 95, 172502 (2005).

[17] B. Borasoy, R. Nissler, and W. Weise, Eur. Phys. J. A 25, 79 (2005).

[18] B. Borasoy, U. G. Meissner, and R. Nissler, Phys. Rev. C 74, 055201 (2006).

[19] J. A. Oller, Eur. Phys. J. A 28, 63 (2006).

[20] T. Hyodo, D. Jido, and A. Hosaka, Phys. Rev. C 78,
PHYSICAL REVIEW D 79, 054004 (2009)

TABLE XXII. $I=1, J=5 / 2, S=0, C=1$. Baryon-meson states with more than one $c$ quark have not been included.

\begin{tabular}{lcccccc}
\hline \hline & $\Delta D^{*}$ & $\sum_{c}^{*} \rho$ & $\Sigma_{c}^{*} \omega$ & $\Sigma^{*} D_{s}^{*}$ & $\sum_{c}^{*} \phi$ & $\Xi_{c}^{*} K^{*}$ \\
\hline$\Delta D^{*}$ & 0 & 2 & $\sqrt{8}$ & 0 & 0 & 0 \\
$\sum_{c}^{*} \rho$ & 2 & 0 & $\sqrt{8}$ & 0 & 0 & 0 \\
$\sum_{c}^{*} \omega$ & $\sqrt{8}$ & $\sqrt{8}$ & 2 & 0 & 0 & 0 \\
$\sum^{*} D_{s}^{*}$ & 0 & 0 & 0 & 0 & -2 & $\sqrt{8}$ \\
$\sum_{c}^{*} \phi$ & 0 & 0 & 0 & -2 & 0 & $-\sqrt{8}$ \\
$\Xi_{c}^{*} K^{*}$ & 0 & 0 & 0 & $\sqrt{8}$ & $-\sqrt{8}$ & 2 \\
\hline \hline
\end{tabular}

TABLE XXIII. $I=2, J=5 / 2, S=0, C=1$. Baryon-meson states with more than one $c$ quark have not been included.

\begin{tabular}{lll}
\hline \hline & $\Delta D^{*}$ & $\sum_{c}^{*} \rho$ \\
\hline$\Delta D^{*}$ & 0 & $\sqrt{12}$ \\
$\sum_{c}^{*} \rho$ & $\sqrt{12}$ & 4 \\
\hline \hline
\end{tabular}

025203 (2008).

[21] D. Jido, A. Hosaka, J. C. Nacher, E. Oset, and A. Ramos, Phys. Rev. C 66, 025203 (2002).

[22] D. Jido, J. A. Oller, E. Oset, A. Ramos, and U. G. Meissner, Nucl. Phys. A725, 181 (2003).

[23] C. Garcia-Recio, M. F. M. Lutz, and J. Nieves, Phys. Lett. B 582, 49 (2004).

[24] T. Hyodo, S. I. Nam, D. Jido, and A. Hosaka, Phys. Rev. C 68, 018201 (2003).

[25] S. I. Nam, H. C. Kim, T. Hyodo, D. Jido, and A. Hosaka, J. Korean Phys. Soc. 45, 1466 (2004).

[26] D. W. Thomas, A. Engler, H. E. Fisk, and R. W. Kraemer, Nucl. Phys. B56, 15 (1973).

[27] S. Prakhov et al. (Crystall Ball Collaboration), Phys. Rev. C 70, 034605 (2004).

[28] V. K. Magas, E. Oset, and A. Ramos, Phys. Rev. Lett. 95, 052301 (2005).

[29] E. E. Kolomeitsev and M. F. M. Lutz, Phys. Lett. B 585, 243 (2004).

[30] S. Sarkar, E. Oset, and M. J. Vicente Vacas, Nucl. Phys. A750, 294 (2005); A780, 78(E) (2006).

[31] S. Sarkar, E. Oset, and M. J. Vicente Vacas, Phys. Rev. C 72, 015206 (2005).

[32] L. Roca, S. Sarkar, V. K. Magas, and E. Oset, Phys. Rev. C 73, 045208 (2006).

[33] T. Hyodo, S. Sarkar, A. Hosaka, and E. Oset, Phys. Rev. C 73, 035209 (2006); 75, 029901(E) (2007).

[34] M. Doring, E. Oset, and S. Sarkar, Phys. Rev. C 74, 065204 (2006).

[35] M. Doring, E. Oset, and D. Strottman, Phys. Rev. C 73, 045209 (2006).

[36] M. Doring, E. Oset, and D. Strottman, Phys. Lett. B 639, 59 (2006).

[37] C. García-Recio, J. Nieves, and L. L. Salcedo, Phys. Rev. D 74, 034025 (2006).

[38] C. García-Recio, J. Nieves, and L. L. Salcedo, Eur. Phys. J. A 31, 499 (2007); 31, 540 (2007). 
[39] H. Toki, C. Garcia-Recio, and J. Nieves, Phys. Rev. D 77, 034001 (2008).

[40] L. Roca, E. Oset, and J. Singh, Phys. Rev. D 72, 014002 (2005).

[41] M. F. M. Lutz and E. E. Kolomeitsev, Nucl. Phys. A730, 392 (2004).

[42] M. Artuso et al. (CLEO Collaboration), Phys. Rev. Lett. 86, 4479 (2001).

[43] R. Mizuk et al. (Belle Collaboration), Phys. Rev. Lett. 94, 122002 (2005).

[44] R. Chistov et al. (Belle Collaboration), Phys. Rev. Lett. 97, 162001 (2006).

[45] B. Aubert et al. (BABAR Collaboration), Phys. Rev. Lett. 97, 232001 (2006).

[46] B. Aubert et al. (BABAR Collaboration), Phys. Rev. Lett. 98, 012001 (2007).

[47] R. Mizuk et al. (Belle Collaboration), Phys. Rev. Lett. 98, 262001 (2007).

[48] L. Tolos, J. Schaffner-Bielich, and A. Mishra, Phys. Rev. C 70, 025203 (2004).

[49] M. F. M. Lutz and E. E. Kolomeitsev, Nucl. Phys. A730, 110 (2004).

[50] J. Hofmann and M.F. M. Lutz, Nucl. Phys. A763, 90 (2005).

[51] J. Hofmann and M.F. M. Lutz, Nucl. Phys. A776, 17 (2006).

[52] T. Mizutani and A. Ramos, Phys. Rev. C 74, 065201 (2006).

[53] N. Isgur and M. B. Wise, Phys. Lett. B 232, 113 (1989).

[54] M. Neubert, Phys. Rep. 245, 259 (1994).

[55] A. V. Manohar and M.B. Wise, Heavy Quark Physics, Cambridge Monographs on Particle Physics, Nuclear Physics and Cosmology Vol. 10 (Cambridge University Press, Cambridge, UK, 2000).

[56] C. García-Recio, J. Nieves, and L. L. Salcedo, Phys. Rev. D 74, 036004 (2006).
[57] W.-M. Yao et al., J. Phys. G 33, 1 (2006).

[58] J. C. Carter, J. J. Coyne, and S. Meshkov, Phys. Rev. Lett. 14, 523 (1965); 14, 850(E) (1965).

[59] M. Artuso et al. (CLEO Collaboration), Phys. Rev. Lett. 95, 251801 (2005).

[60] M. Artuso et al. (CLEO Collaboration), Phys. Rev. Lett. 99, 071802 (2007).

[61] K. M. Ecklund et al. (CLEO Collaboration), Phys. Rev. Lett. 100, 161801 (2008).

[62] B. Aubert et al. (BABAR Collaboration), Phys. Rev. Lett. 98, 141801 (2007).

[63] K. C. Bowler et al. (UKQCD Collaboration), Nucl. Phys. B619, 507 (2001).

[64] D. Ebert, R. N. Faustov, and V. O. Galkin, Mod. Phys. Lett. A 17, 803 (2002).

[65] C. Albertus, E. Hernandez, J. Nieves, and J. M. VerdeVelasco, Phys. Rev. D 71, 113006 (2005).

[66] J. A. Oller and E. Oset, Phys. Rev. D 60, 074023 (1999).

[67] J. Nieves and E. Ruiz Arriola, Phys. Lett. B 455, 30 (1999).

[68] J. Nieves and E. Ruiz Arriola, Nucl. Phys. A679, 57 (2000).

[69] S. Capstick and N. Isgur, Phys. Rev. D 34, 2809 (1986).

[70] N. Isgur and M. B. Wise, Phys. Rev. Lett. 66, 1130 (1991).

[71] H. Y. Cheng and C. K. Chua, Phys. Rev. D 75, 014006 (2007).

[72] X.H. Zhong and Q. Zhao, Phys. Rev. D 77, 074008 (2008).

[73] X. G. He, X. Q. Li, X. Liu, and X. Q. Zeng, Eur. Phys. J. C 51, 883 (2007).

[74] G. E. Baird and L. C. Biedenharn, J. Math. Phys. (N.Y.) 5, 1723 (1964).

[75] V. Rabl, G. J. Campbell, and K. C. Wali, J. Math. Phys. (N.Y.) 16, 2494 (1975).

[76] J. J. de Swart, Rev. Mod. Phys. 35, 916 (1963). 